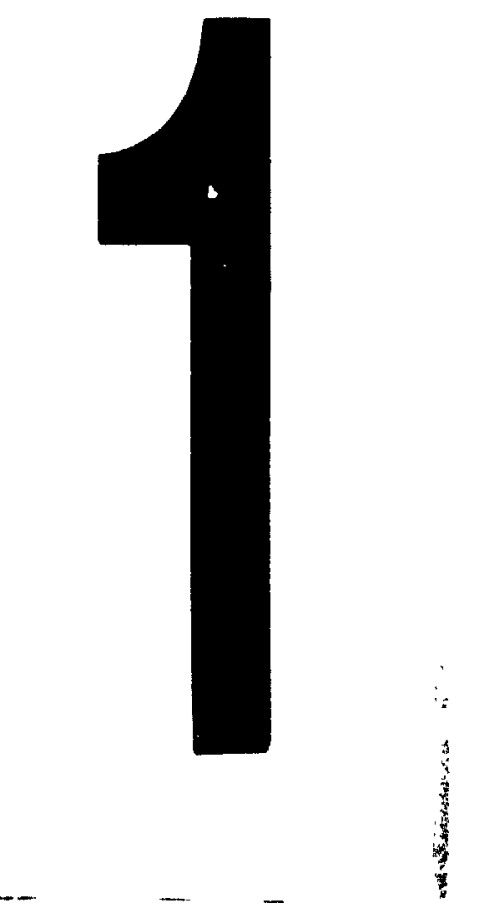

PM-1 3\%" $\times 4 "$ PHOTOGRAPHIC MICROCOPY TARGET MAS 1010. ANSI/ISO "Z2 EOUNALENT

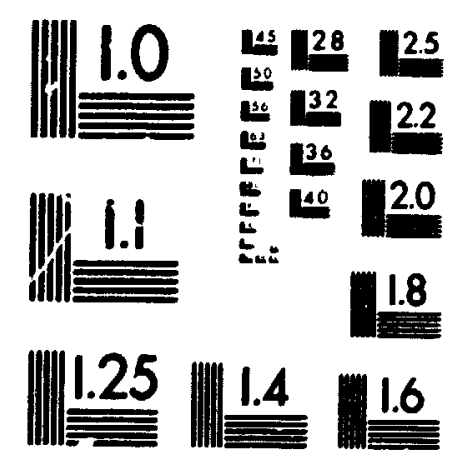

PRECISIONEM RESOLUTION TARGETS

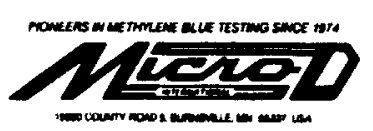

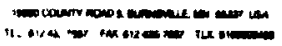




\section{National Library \\ of Canada}

Canadian Theses Service

Otawa, Canada

KIA ON4
Bibliotheque nationale

du Canada

Service des theses canadiennes
NOTICE

The quality of this ricroform is heavily dependent upon the quality of the original thesis submitted for microfilming. Every effort has been made to ensure the highest quality of reproduction possible.

If pages are missing. contact the university which granted the degree.

Some pages may have indistinct print especially if the original pages were typed with a poor typewriter ribbon or if the university sent us an inferior photocopy.

Reproduction in full or in part of this microlorn is governed by the Canadian Copyright ACt, R.S.C. 1970, c. C-30, and subsequent amendments.

\begin{abstract}
AVIS
La qualité de cette rnicroforme dépend grandement de la qualitê de la thèse soumise au microlilmage. Nous avons tout lait pour assurer une qualité supérieure de reproduc. tion.

S'il manque des pages, veuillez communiquer avec ru-iversilé qui a conféré le grade.

La qualité d'impression de certaines pages peut laisse: à désirer, surtuut si les pages originales ont été dartylogra. phiées a laide d'un ruban usé ou sị l'université nous a lail parvenir une photocopie de qualité intérieure.
\end{abstract}

La reproduction, meme partislle. de cefte microforme est soumise a la Loi canadienne sur le droit d'auteur. SRC 1970, c. C-30, et ses amendements subséquents 


\title{
Network Management Standards from the Fault Management Perspective
}

\author{
by
}

William Theodore Aitken, B.Eng.

\author{
A thesis submitted to the \\ Faculty of Graduate Studies and Research \\ in partial fulfilment of the requirements \\ for the degree of \\ Master of Engineering \\ Department of Systems and Compurer Engineering, \\ Carleton University, \\ Ottawa, Ontario, Canada
}

February 29, 1992

(c) William Theodore Aitken 1992 
Canadian Theses Service Service des theses canadiennes

Ouswa Canso

Kia ONA

The author has granted an irrevocable nonexclusive licence allowing the National Libren of Cenada to reproduce, ben, cistribute or sell copies of his/her thesis by any means and in any form or format, making this thesis avallable to interested persons.

The author retains ownership of the copyright in his/her thesis. Neither the thesis nor substantial extracts from it may be printed or otherwise reproduced without his/her permission.
L'auteur a accordé une ficence irrérocable et non exclusive permettant a la Ebliotheque nationale du Canada de reproduire, proter. cistribuer ou vendre des coples de ea these de quelque maniere et sous quelque forme que ce soit pour mettre des exemplaires de cette thèse à la disposition des personnes intéressées.

L'auteur conserve la propriété du droit d'auteur qui protége sa these. Nila these ni des extraits substantiels de celle-ci ne doivent otre imprimés ou autrement reproduits sans son autorisation. 
The undersigned hereby recommend to the Faculty of Graduate Studies and Research acceptance of this thesis

\section{Network Management Standards from the Fault Management Perspective}

submitted by William Theodore Aitken, B. Eng.

in partial fulfilment of the requirements for the degree of Master of Engineering

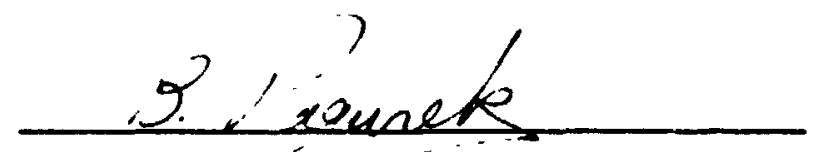

Thesis Co-supervisor

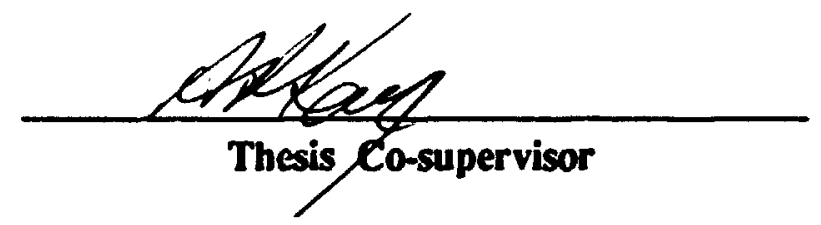

Chair, Department of Systems and Computer Engineering

Carleton University

March 14, 1992 


\section{Abstract}

It is very difficult at this time for universities to become knuwledgeable of and to keep pace with evolving international standards. This thesis examines and evaluates the evolving ISO Network Management (NM) standards from the perspective of the fault management function as the answer to the network maragement problem in an example network environment. As well, this thesis serves as a tutorial on and reference to the latest available ISO and DARPA NM standards documents and termil.:logy. In doing so, it compares the ISO and DARPA NM approaches. Certainly, network management is a complex and difficult problem, and lags behind research in other areas of networking. 


\section{Acknowledgements}

I would like to thank my thesis supervisors, Dr. B. Pagurek and Dr. A.R. Kaye, for suggesting this research and for their guidance on this thesis. Thanks also to the Telecommunications Research Institute of Ontario (TRIO) which provided financial support for this research. My heart felt thanks to Susan for her support. Finally, my love to my mother. 


\section{Carleton University \\ Ottawa, Canada K1S5B6}

Text complete; leaf iv omitted in numberinr. 


\section{Contents}

Abstract ii

Acknowledgements

List of Figures $\quad$ ix

1 Introduction 1

1.1 Background for the Research $\ldots \ldots \ldots \ldots \ldots \ldots \ldots$

1.2 The Network Management Problem . . . . . . . . . . 2

1.3 The Network Management Solution . . . . . . . . . . 5

1.4 Thesis Objectives and Outline $\ldots \ldots \ldots \ldots \ldots \ldots \ldots$

2 OSI Management Standards $\quad 8$

2.1 The Organization $\ldots \ldots \ldots \ldots \ldots \ldots \ldots \ldots$

2.2 The Standardization Process $\ldots \ldots \ldots \ldots \ldots \ldots \ldots$

2.3 Current OSI Management Standards $\ldots \ldots \ldots \ldots \ldots \ldots \ldots 11$

2.3.1 Relationship between OSI Standards . . . . . . . . . . 11

2.3.2 The Architecture . . . . . . . . . . . . . . . 14

2.3.2.1 Management Framework: ISO/IEC 7498-4 . . . . . . . 14

2.3.2.2 System Management Overview (SMO): DIS $10040 \ldots \ldots 17$

2.3.3 Services and Protocols . . . . . . . . . . . . . . 20

2.3.3.1 Common Management Information Protocol (CMIP) $\ldots .20$

2.3.3.2 Common Management Information Services (CMIS) $\ldots .21$ 
2.3.4 System Management Functions (SMFs): $10164-x \ldots \ldots$. . . 25

2.3.4.1 Object Management Function (OMF): DIS 10164-1 f . . 29

2.3.4.2 State Management Fi:nction (STMF): DIS 10164-2 . . . . 30

2.3.4.3 Attributes for Representing Relationships (ARR): DIS

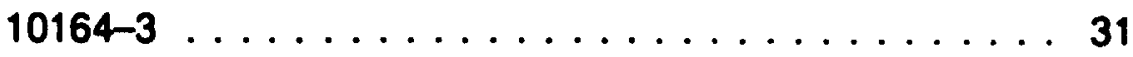

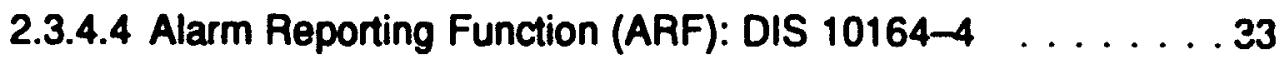

2.3.4.5 Event Reporting Function (ERF): DIS 10164-5 . . . . . 38

2.3.4.6 Log Control Function (LCF): DIS $10164-6 \ldots \ldots$. . . . 41

2.3.4.7 Test Management Function (TMF): CD 10164-12 . . . . 42

2.3.4.8 Confidence and Diagnostic Test Classes (CDTC): 10164-z . 45

2.3.5 Structure of Management Information (SMI) $\ldots \ldots \ldots \ldots$. . 47

2.3.5.1 Abstract Syntax Notation One (ASN.1): ISO $8824 \ldots \ldots 48$

2.3.5.2 Management Information Model (MIM): DIS 10165-1 f . .54

2.3.5.3 Definition of Management Information (DMI): DIS 10165-2 .63

2.3.5.4 10165-3: Definition of Management Attributes . . . . . . .66

2.3.5.5 Guidelines for the Definition of Managed Objects (GDMO): DIS

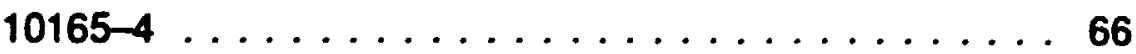

3 DARPA Management Standards $\quad 74$

3.1 The Organization . . . . . . . . . . . . . . . 74

3.2 The Standardization Process $\ldots \ldots \ldots \ldots \ldots \ldots \ldots 77$

3.2.1 RFC1052: . . . . . . . . . . . . . . .78 


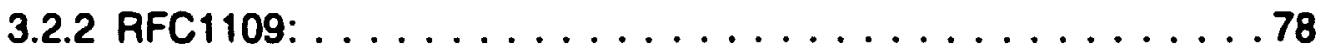

3.3 Current DARPA Management Standards . . . . . . . . . . 79

3.3.1 Services and Protocols . . . . . . . . . . . . . . 81

3.3.1.1 RFC1157: SNMP $\ldots \ldots \ldots \ldots \ldots \ldots \ldots$. . .

3.3.2 DARPA Structure of Management Information (SMI) $\ldots \ldots$. . 87

3.3.2.1 Abstract Syntax Notation One (ASN.1): ISO $8824 \ldots . .87$

3.3.2.2 RFC1155:Structure of Management Information . . . . . 87

3.3.2.3 RFC1156: MIB-I $\ldots \ldots \ldots \ldots \ldots \ldots \ldots \ldots \ldots$

3.3.2.4 RFC1213: (MIB-11) $\ldots \ldots \ldots \ldots \ldots \ldots \ldots \ldots$

3.3.2.5 RFC1214: OIM-MIB $\| \ldots \ldots \ldots \ldots \ldots \ldots$

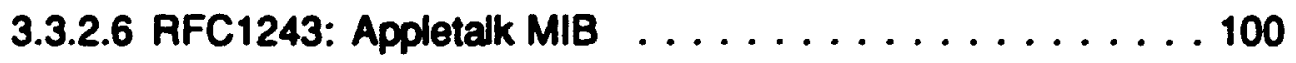

4 Comparing OSI and DARPA 104

4.1 Comparing OSI and DARPA Protocol Stacks ... . . . . 104

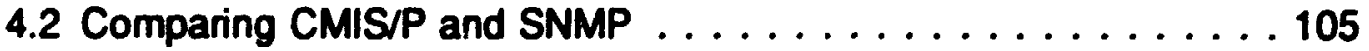

$\begin{array}{ll}5 \text { Fault Management } & 118\end{array}$

5.1 The Fault Management (FM) SMFA . . . . . . . . . 118

5.1.1 Fault Management Requirements . . . . . . . . . . 119

5.1.2 Fault Management Functions . . . . . . . . . . . . 120

5.2 A Third Generation Network Environment . . . . . . . . . . 121 
5.3 The Fault Management Solution . . . . . . . . . . . 132

5.3.1 Previous Fault Management Approaches . . . . . . . . . 132

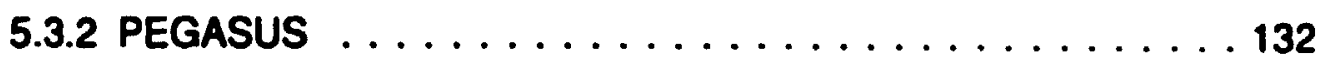

5.3.3 Applying OSI STANDARDS to NETWORK MANAGEMENT (NM) $\ldots \ldots \ldots \ldots \ldots \ldots \ldots \ldots \ldots \ldots \ldots \ldots \ldots \ldots \ldots$

5.3.4 Applying OSI STANDARDS to the Representation of NETWORK ELEMENTS . . . . . . . . . . . . . 143

6 Research Summary and Future Research 167

6.1 Research Summary . . . . . . . . . . . . . . . . 167

6.2 Future Research $\ldots \ldots \ldots \ldots \ldots \ldots \ldots \ldots \ldots \ldots$

6.2.1 Object-Oriented Human-Machine Interface (HMI) $\ldots \ldots \ldots 173$

6.2.2 Data Base Management Systems (DBMS) $\ldots \ldots \ldots \ldots 175$

6.2.3 Analysis Functions . . . . . . . . . . . . . . 179

6.2.4 OSI Network Management Development Ervironment . . . . . 181

6.2.5 The Future of Network Management in the 90s . . . . . 183

$\begin{array}{ll}\text { Appendix A Glossary } & 195\end{array}$

Appendix B June 1991 ISONEC Developments 219

Appendix C Previous Implementations 225

$\begin{array}{ll}\text { Bibliography } & 229\end{array}$

Index 241 


\section{List of Figures}

Figure 1

Figure 2

Figure 3

Figure 4

Figure 5

Figure 6

Figure 7

Figure 8

Figure 9

Figure 10

Figure 11

Figure 12

Figure 13

Figure 14

Figure 15

Figure 16

Figure 17

Figure 18

Figure 19
The Network Management Problem ... . . . . . 4

Rolationship between OSi Standards (by title) . . . 12

Relationship batween OSI Standards (by 150

reforence) ..................... 13

Notwork Management Information Exchange . . . . . 15

Syatoms Management Interactions . . . . . . . . . 18

Example of Clms Use . . . . . . . . . . . . 24

Rolationship of SMFAs and SMFs (by titie) . . . . . . . 26

Rolutionship of SMFAs and SMFs (by 150 reference) .27

Example Event Report Function . . . . . . . . . . 40

$\log$ Modd .....................42

Test Modet . . . . . . . . . . . . . . . . . . . .44

Example of ASN.1 Object Identifier Tree . . . . . . . 54

ASN.t of TOP Managed Object Class . . . . . . . . .64

ASN.1 of TOP Managed Object Class (cont.) . . . . . . 65

Relationship Botween DARPA Standards . . . . . . . 80

Comparing OSI and DARPA Protocol Stacks . . . . 104

Comparing OSt and DARPA . . . . . . . . . 117

Sample Network Environmant . . . . . . . . . . . 122

Bridge Functionality . . . . . . . . . . . . . . . 124 
Figure 20

Figure 21

Figure 22

Figure 23

Figure 24

Figure 25

Figure 26

Figure 27

Figure 28

Figure 29

Figure 30

Figure 31

Figure 32

Figure 33

Figure 34

Figure 35

Figure 36

Router Functionality . . . . . . . . . . . . . . 125

Cateway Functionality . . . . . . . . . . . . . 127

Sample Fault Management Environment . . . . . . 128

Components of Interoperable Management Open

Systems . . . . . . . . . . . . . . . 138

Interim Notwork Management (INM) using CMIP . . 140

IEEE 802 LAN Bridge Managed Objects . . . . . . . 144

IFEE 802 LAN Bridge Managed Objects (cont.) . . . 145

IEEE 802 LAN Bridge Name Bindings . . . . . . . . 146

IEEE 802.1 Containment . . . . . . . . . . . . . 147

IEEE 802.1 Class Inheritance . . . . . . . . . . . . 150

IEEE 802.1 Class Registration . . . . . . . . . 151

IEEE 802.3 Hub Managed Objects . . . . . . . . . . . 154

IEEE 802.3 Hub Managed Objects (cont.) . . . . . . 155

IEEE 802.3 Hub Port mo Class . . . . . . . . . . 156

OIW NMSIG Computer System . . . . . . . . . . . . 159

Alarm Record Class Inheritance . . . . . . . . . . 178

Multiaxial Proposal . . . . . . . . . . . . . . 180 


\section{CHAPTER 1 Introduction}

\subsection{Background for the Research}

Efficient and effective information technology is crucial for meeting the challenge of organizational prosperity in the 1990 s and early 2000 s. Information technologies that can greatly enhance data and person-to-person communications will have significant impact on organizational change [95]. Communications technologies permit both individuals and computer processes to rapidly transmit and receive information'over distances. Voicemail, electronic mail (E-mail) and FAX increase cooperative inter-personal activities, wh:le data and software standardization offered by Electronic Data Interchange (EDI) and Electronic Fund Transfer (EFT) fill a need for large-scale data exchange between computer processes. At the same time as communications technologies assume a major role in shaping the future viability of organizations, the organizations become dependent upon, and vulnerable to, these same technologies.

Today, many large organizations are involved with and dependent upon communication networks, either as users of public networks or as operators of their own private networks. Already a technology gap exists between user expectations and the communications networks' inability to live up to their promise because of their unmanageability. Thus, the management of communications networks has become an important issue in the communication industry; there is great interest in and considerable pressure for development of common techniques and strategies to effectively manage complex networks. The

\footnotetext{
1 Terme in BOLD are defined in Appendix A: Glowsary and are contuined in the Index.
} 
activities that address this are collectively known as network management. "However, efforts to provide compatible management systems are not progressing at the same rate as the technological advances that drive the industry," [68]. Certainly, network management is a complex and difficult problem, and lags behind research in other areas of networking.

\subsection{The Network Management Problem}

Computer network technology has evolved through three generations: mainframe, departmental and desktop. Simultaneously, the management of computer networks has grown more difficult.

First generation computing was characterized by separate voice and dat? ueivorks, mainframe-centered computing and centralized decision-making. Network management was mainframe-centered with a one-to-one relationship between the service user and the service provider. The topology of these networks was point-to-point and casily managed.

The next generation of computing was characterized by packet switching, heterogeneous computer systems provided by multiple vendors and a more distributed approach to decision-making. The topology was point-to-multipoint logical networks overlaid on top of the physical network. This combination of logical and physical added several orders of magnitude to the complexity of network management.

The third generation of computing ertended the decentralization trend. Its characteristics are local area network (LAN) and client-server technologies linking desktop PC's and workstations into cooperative computing groups. In tum, servers act as access points into the enterprise backbone wide area network (WAN). Currently, the proliferation 
of workstations, LANs and their decentralized administration make the task of network management even more difficult.

At the same time, other factors complicate the issue. The success of the networks leads to heavier traffic which exhausts reserve network capacity and leads to performance bottlenecks. As well, the increasing speed of network media increases the complexity of network management at the same time as it decreases the timeframe to react to network problems. A network manager must be able to monitor the network in order to obtain realtime data on performance and traffic characteristics, diagnose communications problems and reconfigure the network to meet changing needs and environmental conditions. Though vendors increasingly utilize standard communication protocols in their products, many continue to use proprietary solutions for network management. Therefore in heterogeneous environments, users are faced with disparate vendor-specific dispiays and command languages for each class of network component. "The result is a simultaneous increase in cost and reduction in efficiency," [6].

In today's world, users and their management are demanding seamless operation of complex applications over heterogeneous networks, delivered with high availability and reliability. The inverted pyramid in Figure 1 represents the degree of difficulty in providing the capabilities of each building block of the network, as well as the relative benefits provided by each. Network users are not interested in network management architectures. But they do want systems that keep their netwcik running, preferably without requiring much user guidance [11]. Clearly what is needed is a common set of integrated tools by which the separate communications services and resources can be 
managed.

\section{THE P R O B L E M}

- to provide Quality of Service and transparent interoperability in a multi - vendor environment

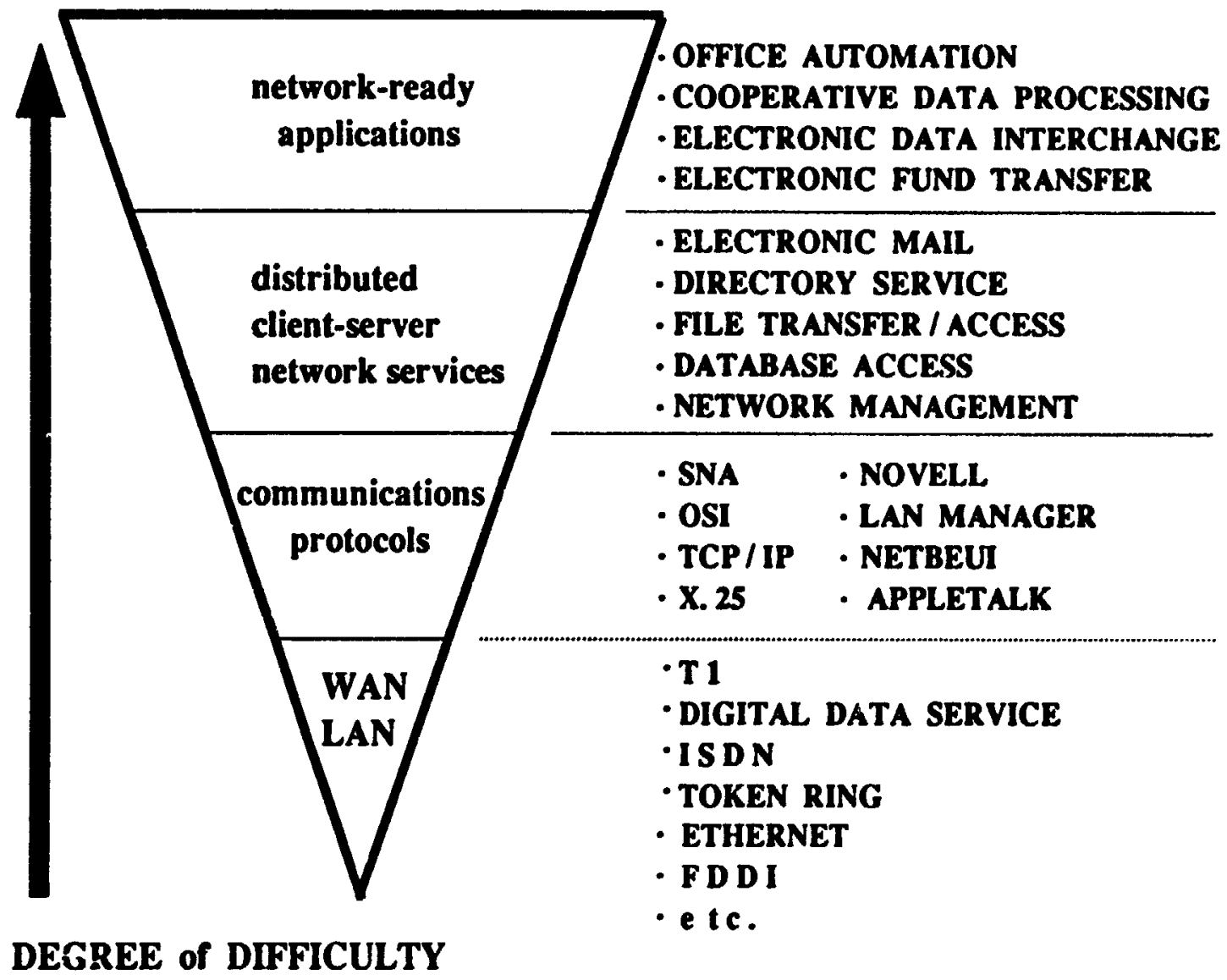

Figure 1 The Network Management Problem 


\subsection{The Network Management Solution}

The emerging solution for network management is a set of agreed to international basic standards with auxiliary functional agreements on implementation and conformance testing of the network management implementations. Proprietary network management systems will not vanish overnight. However, just as the international networking community has adopted the Open Systems Interconnection (OSI) 7-layer Basic Reference Model [34] , the open-networking community is converging on the OSI Management model [37]. This model places a repository of management information, based on object-oriented design, at each network node and manages this information with application layer protocols. Object orientation builds on the foundation of client-server computing [89]. This design principle defines four basic elements of an object. An object class describes objects as being related to one another and able to inherit attributes and data. An object instance is a specific manifestation of a class. A method is the behaviour or operation supported by classes of objects. A link connects two classes or instances of classes and defines relationships between them [84].

OSI management standards are defined in four series of ISO/IEC JTC1 standards

documents: Architectural, Services and Protocols, Management Functions and Structure of Management Information. The goal of these standards is to allow for "management of telecommunications networks which comprise various information processing systems and telecommunication equipment from multiple vendors in an integrated manner," [65]. 


\subsection{Thesis Objectives and Outline}

International standardization activities are conducted by representatives of government and industry. Generally, universities do not participate in the standardization committees in depth. The international scope of the standards results in large volumes of documentation, which are not readily available without incurring considerable expense. Being industry dominated, the implementation of the standards is market-driven and proprietary-oriented. Therefort, it is very difficult at this time for universities to become knowledgeable of and to keep pace with the evolving international standards.

This thesis examines and evaluates the evolving network management standands from the perspective of the fault management function as the answer to the network management problem. The objectives of this thesis are :

- to provide a perspective on a relatively new area in which there is voluminous literature;

- to collect, synthesize and present the current OSI and DARPA network management standards;

- to serve as a tutorial on and reference to the latest available OSI and DARPA network management standards documents and terminology, including a Glossary and an Index;

- to compare the OSI and DARPA network management approaches;

- to describe an example real world network fault management environment to the non-communications specialists of the TRIO-sponsored PEGASUS project [21]; and, 
- to present a possible migration path for the example network from DARPA network management towards OSI network management.

Chapter 2 outlines the ISO standards organization and its process for producing standards. Then the OSI network management framework is described and its system management functions and management information model are explored. This work is a synthesis based on the June 1990 version of the ISO standands documents. In Chapter 3 the DARPA environment is addressed in the same manner, including the latest 1991 Internet standards documents. Chapter 4 provides a comparison between these two philosophically different approaches to the network management problem. A TRIO sponsored application development for network fault diagnosis in a real network is introduced in Chapter 5. Lastly, Chapter 6 presents and discusses the conclusions of this thesis and identifies some issues for the future of network management standards.

To gain a quick overview the reader may wish to follow this suggested first reading guideline:

- Terms in BOLD are defined in Appendix A: Glossary and are contained in the Index.

- Chapter 1 (complete);

- Chapter 2 (2.1, 2.2, 2.3 (to page 28 ), 2.3 .5 (to page 65)) ;

- Chapter 3 (3.1, 3.2, 3.3 (to page 85);

- Chapter 4 (complete, if the comparison is of interest);

- Chapter 5 (5.1, 5.2, 5.3 (to page 150); and,

- Chapter 6 (complete). 


\section{CHAPTER 2 OSI Management Standards}

\subsection{The Organization}

The International Organization for Standardization (ISO) and the International Electrotechnical Commission (IEC) form the specialized system for worldwide standardization. Their members are the national standards bodies in more than 90 countries. The large range of information technology standardization is focused in the ISO/IEC Joint Technical Committee on information technology, normally referred to by its short name, JTC 1 . This committee, with its 17 sub-committees and more than 60 active working groups, is a primary forum for voluntary industrywide standardization in the information technology field. Within ISO/IEC JTCl, Working Group 4 of Subcommittee 21 (SC2I/WG4) is responsible for the development of OSI management.

A second international group concerned with OSI management is the International Telegraph and Telephone Consultative Committee (CCITT) of the International Telecommunications Union (ITU), an agency of the United Nations. Within CCITT, Study Group Seven (SG7) is responsible for OSI management. In 1989, CCITT joined ISO/IEC to develop OSI management. Both organizations are committed to producing one set of texts applicable to both organizations. This thesis references the ISO/IEC. document numbers, but corresponding CCITT document numbers are to be defined.

For a specific application, in order to provide a specific function, it is necessary to make a selection of the parameters and options available in OSI standards. For this reason, the previously mentioned standards are known as base standards. Base 
standards are normative standards which tell "what" should be standardized, but not "how" they should be standardized. A new type of standard document, the functional standard defines the combinations required to achieve a specific function. The whole concept of OSI is based on the provision of interoperability between computer systems in a multi-vendor environment and as such interoperability cannot be guaranteed unless there are agreements on the parameters and options to be used. Functire ' standards address "how" the base standards will be implemented and "how" the implementations will define their conformance.

A number of organizations have been established to promote and prepare functional standards. Such organizations include the Corporation for Open Systems (COS) in the United States; the Standards Promotion and Applications Group (SPAG) (the European counterpart to COS); the World Federation of MAP/TOP User Groups, (OSITOP); the Promoting Conference for Open Systems Interconnection (POSI) in Japan; the European Workshop on Open Systems (EWOS); the United States National Institute for Science and Technology (NIST)/OSI Implementors Workshop (OIW); the Asia and Oceania Workshop (AOW); and the OSI/Network Management Forum (the Forum). These organizations are working with ISO/IEC JTC 1 to make available functional profiles which will be published as ISO/IEC International Standardized Profiles (ISPs). Profiles are being defined, as a joint effort of the three Regional Workshop Network Management (RWNM) groups, OIW NMSIG, EWOS EG-NM, and AOW NMSIG. This part of the ongoing liaison/planning activities between the three RWNM groups. The various government OSI procurement specifications, widely known as Government OSI Profiles (GOSIPs) 
are examples of such profiles [69]. In Canada, the Canadian Open Systems Application Criteria (COSAC) are specifications that have been developed to provide guidance to departments and agencies of the Federal government to assist them in cumplying with the OSI implementation policy.

From the vendor perspective, the OSINM Forum, formed in 1988, is currently working on OSI Management implementation agreements. The OSINM Forum, consisting of major vendors and service providers, is dedicated solely to network management and to developing products based on OSI Management even before the standards are completed. COS has been contracted by the OSINM Forum to develop a suite for testing interoperability and conformance [26]. COS, whose membership includes users and nearly all of the major computer vendors, is a nonprofit organization attempting to speed up OSI adoption, especially in the United States (U.S.). It serves as an independent body evaluating vendors' OSI offerings to find out if they conform to OSI standards and interoperate with other OSI systems. Products which pass this inspection are given a "COS mark" as a seal of approval.

\subsection{The Standardization Process}

The standards developed by ISO/IEC can be called de jure standards as the process is one of voting by the international member bodies. When work on a new work item is begun, the Working Group develops a working draft. The working draft is assigned an ISO registration number for document tracing purposes, e.g. $[62$ ]. When there is consensus that the working draft is correct, the Working Group requests that the document 
be submitted for committee approval. It then attains committee draft (CD) status, e.g. [60].

The next step after committee approval is Draft Proposal $(D P)$ status. Before the proposed standard can progress further, all technical issues must be resolved. When the DP is approved, its status is changed to Draft International Standard DIS, e.g. [53]. At this point, it is considered technically stable. When a DIS is approved by at least $75 \%$ of the national bodies voting, it is issued as an International Standard (IS), e.g. [37]. Once approved as an IS it can be changed only by an addendum process which is similar to the one just described [69].

\subsection{Current OSI Management Standards}

\subsubsection{Relationship between OSI Standards}

Network management can be viewed as a set of operational and administrative mechanisms necessary to:

- bring up, enroll and/or alter network resources;

- keep network resources operational;

- fine tune these resources and/or plan for their expansion;

- manage the accounting of their usage; and

- manage their protection from unauthorized use/tampering. 
Figure 2 shows the interrelationships of the OSI standards documents (by title) that have been or are being developed for network management.

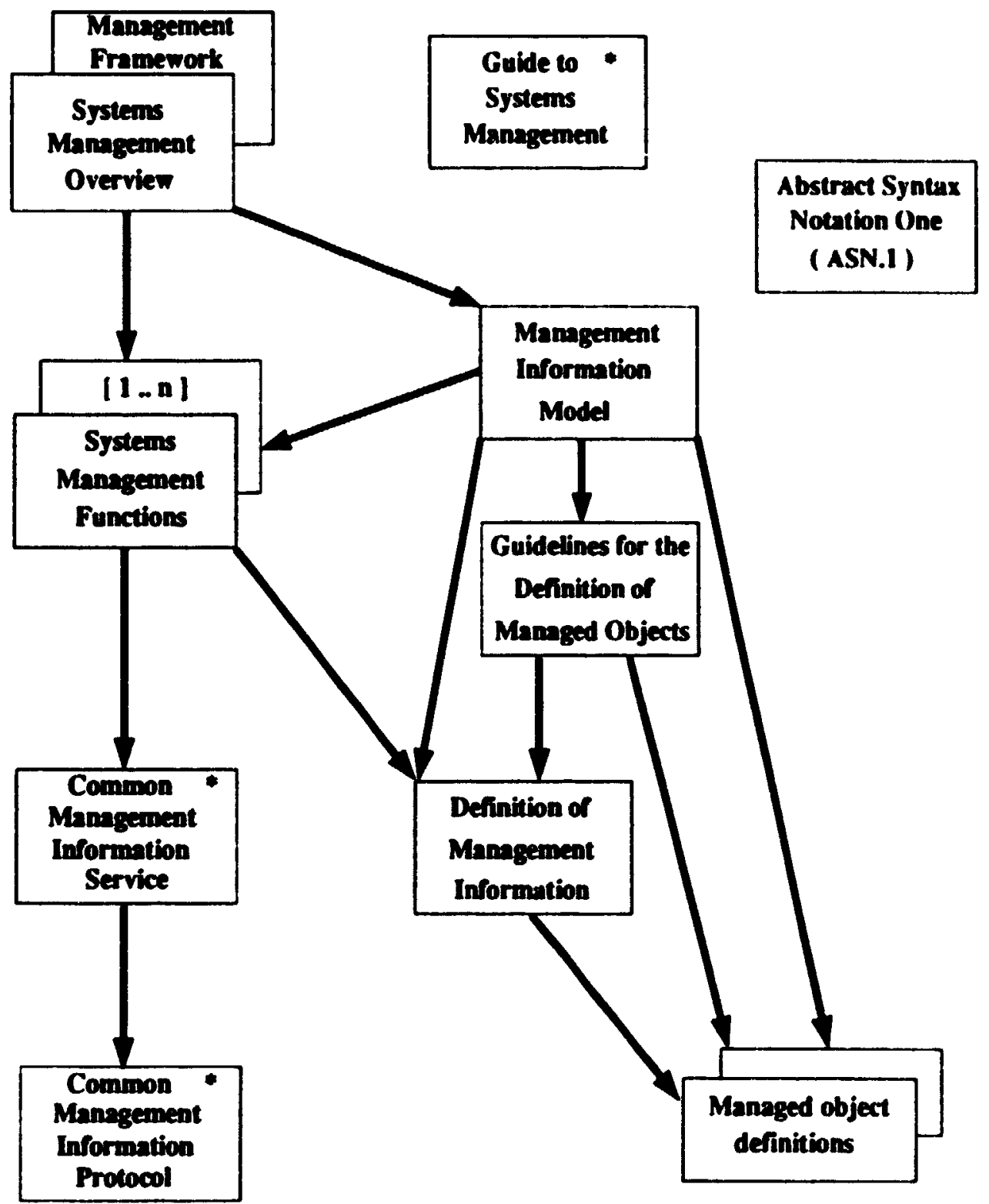

Figure 2 Relationship between OSI Standards (by title) 
Figure 3 shows the same interrelationships of these OSI documents using their ISO reference and commonly known mnemonic name.

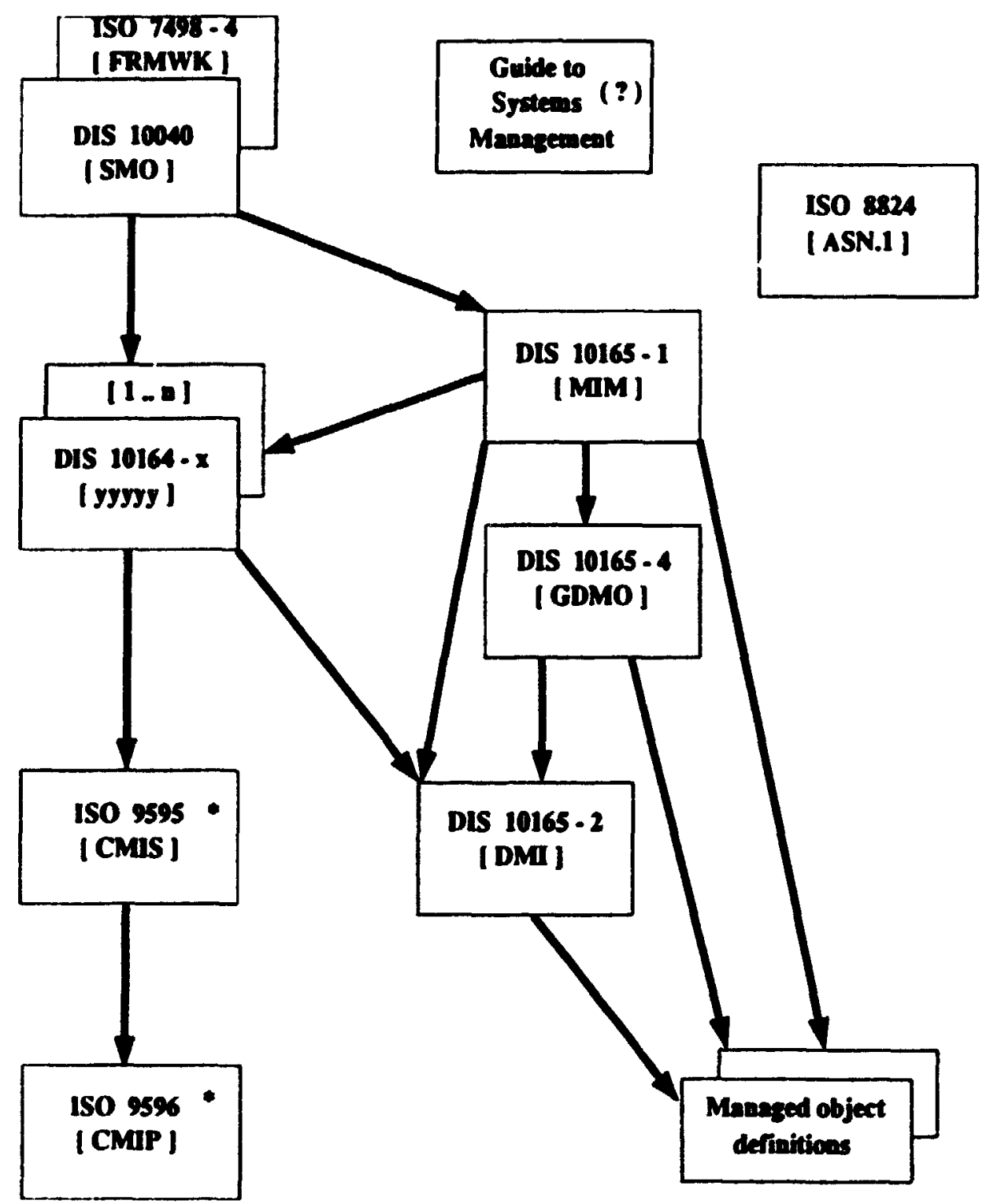

Figure 3 Relationship between OSI Standards (by ISO reference) 
All the documents, except those with an asterix (*) have been acquired for the purposes of this thesis. The Guide to Systems Management is to be developed at some future date and no reference to it was found by this author during this research.

These documents address the elements that are required for the description and standardization of a network management system [99] :

- a model or set of models for understanding management;

- a set of services and related protocols for performing remote management operations;

- a common structure of management information for registering, identifying and defining network resources; and

- detailed specifications of the network resources.

The rest of this section will present the role of each of these standards within OSI network management.

\subsubsection{The Architecture}

2.3.2.1 Management Framework: ISO/IEC 7498-4 [37] This document [37] describes the basic concepts and requirements of OSI management. In order to accomplish management activities, information must be exchanged among open systems. The management model is an extension of the well known OSI 7-layer model [34]. Figure 4 depicts network management as a specific layer 7 application exchanging management information on top of an OSI protocol stack (layer 1-6). 


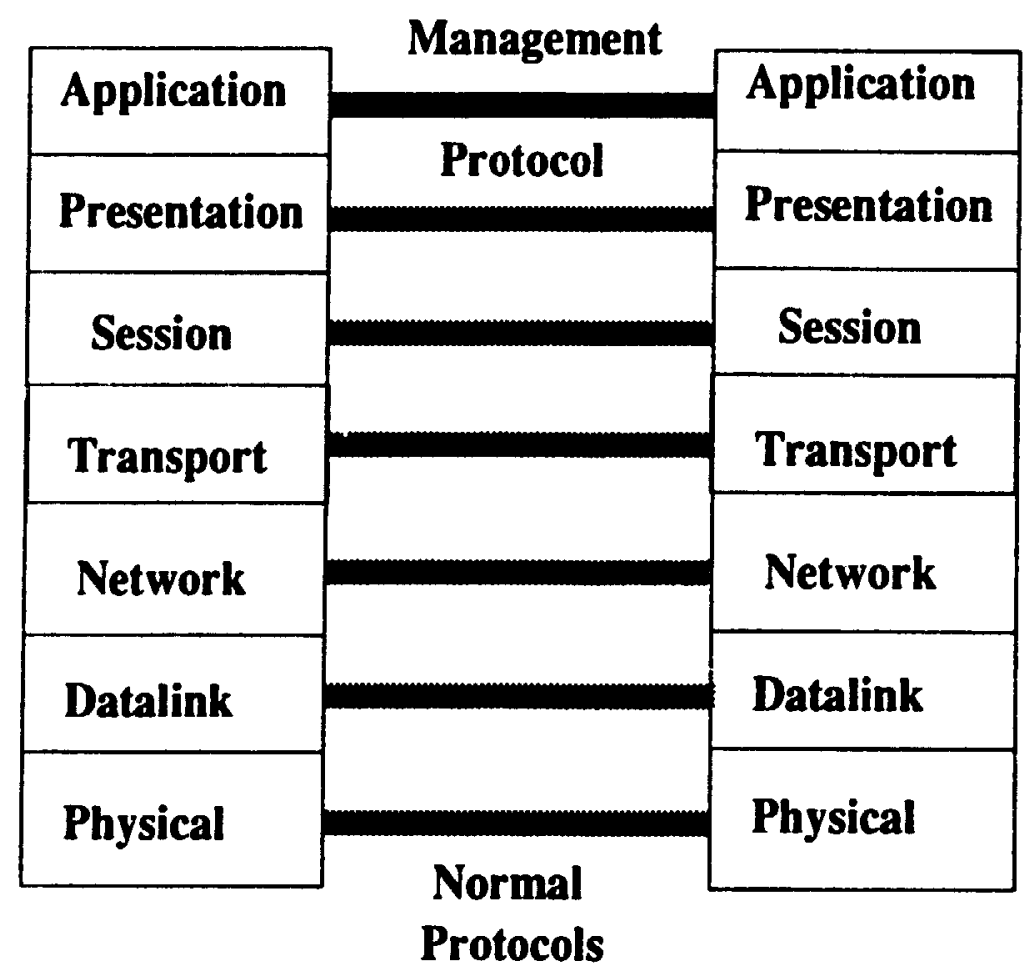

Figure 4 Network Management Information Exchange

OSI management provides the ability to monitor and control network resources, which are represented as managed object (MO). A managed object is an abstracted view of a resource (logical or physical) that represents properties as seen by management. An example of a logical resource is a bridge port table object (see oBridgePortTable in Figure 28 ) which represents the port table actually used by a LAN bridge . An example of a physical resource is a port board in a vendor's LAN bridge. Managed objects are located within a management domain and may be embedded in other managed objects. A management domain is a collection of managed objects for which a common 
administrative policy applies. All information required for network management purposes is defined by managed objects which reside in a Management Information Base (MIB). "The MIB neither restricts the interpretation of management data to a predefined set, nor to whether the data is stored in a processed or unprocessed form," [37]. However, an application layer protocol is employed by network management communications to manipulate MIBs, therefore both the asstract syntax and the semantics of information which is part of the MIB are defined.

Management information is used by the system administration to assist in making management decisions and to communicate those decisions to the system resources. System administration is the external user (person or machine) perspective of systems management. In order to assist in the definition of the information and tools provided, systems management has defined these elements along logical, but arbitrary, task-related or functional boundaries. [37] defines five systems management functional areas (SMFAs) to address these user requirements. These functional areas are designed to allow a modular approach to the design of management functionality. The five areas are:

- accounting management (AM)

- configuration management (CM)

- security management (SM)

- performance management (PM)

- fault management (FM).

Accounting management makes it possible to determine and allocate costs and charges for the use of network resources and to limit the use of those resources. Configuration 
management enables network managers to change the configuration of remote network elements. Security management allows the managing of access control, authentication, encryption, key management and security logs. Performance management provides the facilities to monitor and evaluate the behaviour and effectiveness of the network. Analysis of performance data may be used to initiate diagnostic testing procedures. Fault management [55] provides the abilities to perform fault detection, fault isolation and the correction of abnormal operation. This is the main SMFA covered by this thesis.

\subsubsection{System Management Overview (SMO): DIS 10040 [42]}

This is the architecture document [42] for OSI management which describes information, functional and communications aspects of the model. Management of a communications environment is an information processing application. Because the environment being managed is distributed, the individual components of the management activities are themselves distributed. Management applications perform the management activities in a distributed manner. Two or more systems management application entities may cooperate to provide an instance of a distributed management application. As shown in Figure 5, interactions that take place between systems management application entities are abstracted in terms of system management operations and notifications issued by one entity to the other. Management activities are effected through the manipulation of managed objects which, together with their attributes constitute the system's MIB. 


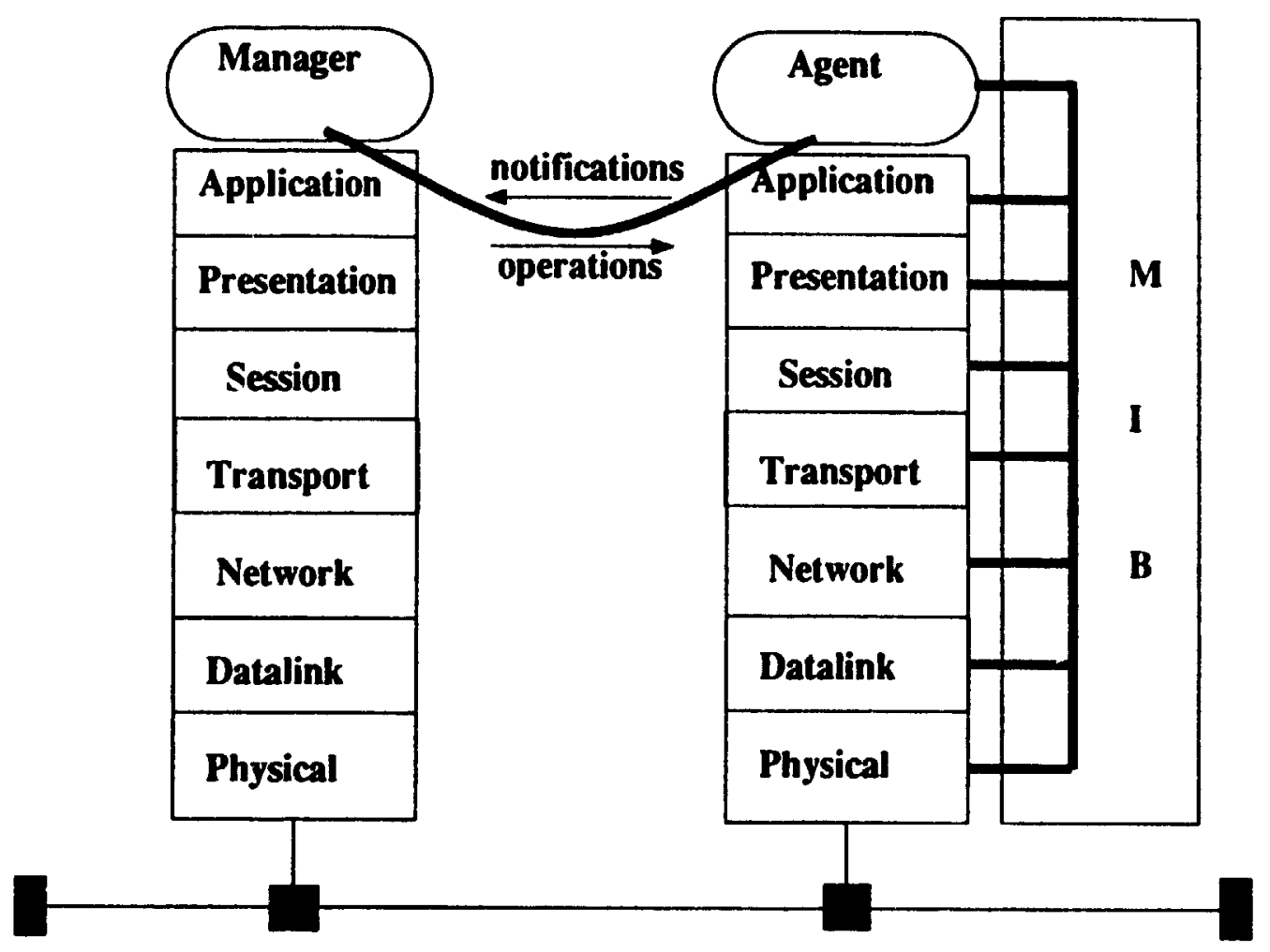

Figure 5 Systems Management Interactions

Information Aspects All managed objects that are visible within the OSI environment and need to be managed are the concern of systems management. Standardized managed objects are expected to be specified by the standardization organizations responsible for standardization of the resources represented by the managed objects (i.e. the group responsible for standardizing a $\mathrm{N}$-layer protocol entity is also responsible for standardizing the managed object that represents the management view of that protocol entity (see 
Figure 5)). A full description of the information model is given in [50]. Guidelines for defining managed objects is given in [52] and a collection of definitions of management information which may be used for such definitions is given in [51].

\section{Functional Aspects}

The concept of user oriented requirements is intended to provide a frame of reference within which requirements for functions may be identified. Each requirement is satisfied by one or more functions. The specification of a systems management function (SMF) defines the management activities and information that are necessary to meet the requirements. Management functions may be combined to accomplish a specific management activity. In general, the agent cannot determine the purpose of the management operations it receives or the notifications it emits. For example, an open system cannot determine whether its responses to read error counters requests will be used for the purpose of fault management or performance management. The agent responds individually to requests from the manager, without needing any wider context within which to carry out the request.

\section{Communications Aspects}

The interactions between a manager and an agent are realized through the exchange of management information using OSI application layer protocols. The general OSI communication service concerning management operations and notifications for systems management is described in [38]. Systems management services mirror the exchange defined at the managed object boundary and provide additional support for the identifi- 
cation of managed objects by filtering and for control of communications. There is strict correspondence between the types of exchange defined in the information model at the managed object boundary and communications support in systems management services.

\subsubsection{Services and Protocols}

Network management requires the ability to communicate management information and commands between open systems. The management communication protocol and service provide an information transfer mechanism, mutually agreed by peer participating manageme:It entities. The service and protocol developed by ISO/IEC for OSI systems mans.jement are the Common Management Information Service (CMIS) [38] and the Common Management Information Protocol (CMIP) [39]. CMIP is the application layer protocol used in the OSI environment to transfer management commands and information between open systems. CMIP specifies the makeup of the management messages, while CMIS specifies the service interface to CMIP. Although not stated in these particular standards, normally in any example of management communication, the management entity on one end of the association assumes a manager role, while the peer management entity on the other end of the association assumes the agent role.

\subsubsection{Common Management Information Protocol (CMIP) [39]}

This document [39] is specification of the protocol which is used to provide the CMIS service. CMIP provides a commonly understood format for the transfer of management information and commands between the peer management entities. Typically, one of the peers acts in a manager role and one acts in an agent role during management 
communications. CMIP specifies the generic transfer mechanism needed by the systems management function. The types of management information and commands that are exchanged using CMIP take the general form of event notifications, information or action requests, and responses, containing either the requested information or some indication as to why the request failed. The detail of CMIP is outside the scope of this thesis.

\subsubsection{Common Management Information Services (CMIS) [38]}

This standard defines Common Management Information Services (CMIS) [38], a service to convey object-oriented management messages between open systems. CMIS provides a full range of services by means of a smaller number of basic service primitives. The service primitives are:

- M-INITIALIZE service: to establish an association with another CMIS user for the purpose of exchanging management information.

- M-EVENT-REPORT service: to report an event about a managed object to another CMIS user.

- M-GET service: to request retrieval of management information (attribute values of managed objects).

- M-SET service: to request modification of management information (attribute values of managed objects).

- M-ACTION service: to request another service user to perform an action (e.g. start test, deenroll) upon a certain managed object. 
- M-CREATE service: to create a new object instance, supplying all necessary attribute values.

- M-DELETE service: to delete existing object instances.

- M-TERMNATE service: to release an association orderly.

- M-ABORT service: to release an association abruptly.

The intent of the CMIS service primitives is to allow the management service user:

- to specify the management operation (command) being transmitted;

- to pass appropriate support information;

- to pass result or error information;

- to identify the particular managed object/attribute/action/event to be operated upon, either by directly naming the specific object instance or by specifying criteria by which an appropriate set of objects can be selected (i.e., through the use of "scoping". to select potential object targets, and "filtering," to select the actual targets which satisfy a specified set of cond:tinncl.

- to identify the particular insisi.. ment communication;

- to pass access control inf... .3 ation; and,

- to specify when operations and events wccurred.

CMIS capabilities are partitioned into functional units which can be negotiated between peer management applications communicating over a given application association. The resulting negotiated agreement limits the range of management communication allowed on that association. The kemel functional unit of CMIS includes all the basic service primitives mentioned above. This kernel functionality can be augmented by ne- 
gotiating functional units which make available services within CMIS for "scoping" and "filtering," in order to designate sets of objects to be operated on and to allow for multiple replies in such cases. An additional functional unit makes available the extended use of presentation layer services (layer 6).

Figure 6 is an example of the use of CMIS service primitives communicated between a manager and an agent. The black dots within the agent represent managed objects. These managed objects contain the administrative and operational state attribute-value pairs. This example illustrates:

- the confirmed creation of a circuit object (1. and 2.);

- setting it administratively for use (3. and 4.);

- at some later time having the fact that the physical circuit failed (5.); and,

- being reported as an event report for management action (6.) 


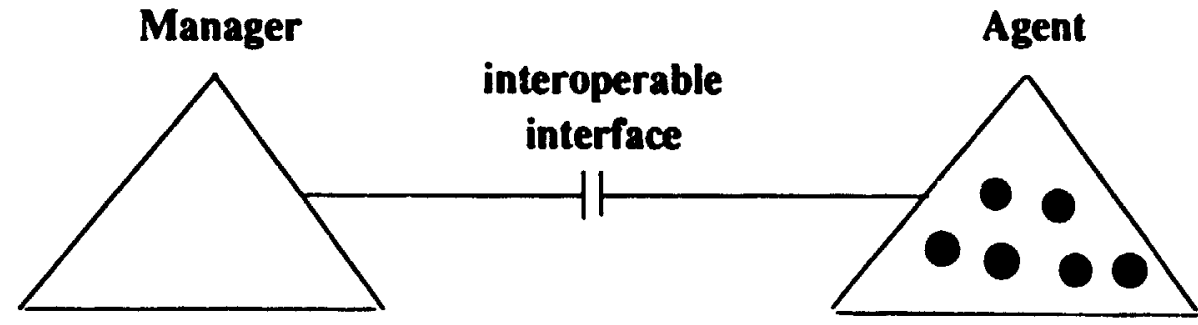

$\begin{array}{lll}\begin{array}{l}\text { 1. Request } \\ \text { Circuit } \\ \text { object }\end{array} & \text { M-Create request } & \text { M-Create response } \\ & \text { M-Event-Report request } & \text { 2. Create } \\ \text { (creation event ) } & \text { Management } \\ \text { View (object) }\end{array}$

3. Make object $\longrightarrow$ M-Set request $\longrightarrow$. Allow Use Available (administration state= unlocked)

for Use

$\longleftarrow$ M-Event -Report request (attribute change event, adminstrative state $=$ unlocked)

6. Process

Failure (transmission alarm event)

5. Circuit

Reports

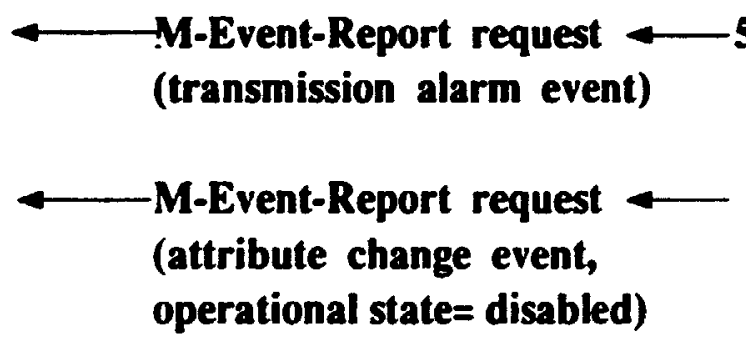

Fails

Figure 6 Example of CMIS Use 


\subsubsection{System Management Functions (SMFs): 10164-x}

In general, there is a many-to-many relationship between functions and requirements. Since the five SMFAs have overlapping requirements, decomposing them produces redundancy. Management functions and management information applicable to one SMFA are often applicable to other SMFAs. Decoupling a function from a specific SMFA led to the development of a set of management functions that is referred to as the System Management Functions (SMFs) (10164-x). They provide a generic platform of common network managemert capabilities available to any management application [79]. Each SMF defines a set of services that it can provide. Figure 7 depicts the functional relationship of SMFAs and SMFs (by title). Some of these standards are at the DIS stage while others are still at the CD stage. Figure 8 shows the same relationship using their ISO reference and commonly known mnemonic name. All of the cited documents have been acquired for the purposes of this thesis.

There are currently seven SMF at DIS status:

- Object Management [OMF] [43];

- State Management [STMF] [44];

- Attributes For Representing Relationships [ARR] [46];

- Alarm Reporting [ARF] [46];

- Event Report [ERF] [47]:

- Log Control [LCF] [48]; and

- Security Alarm Reporting [SARF] [49]. 


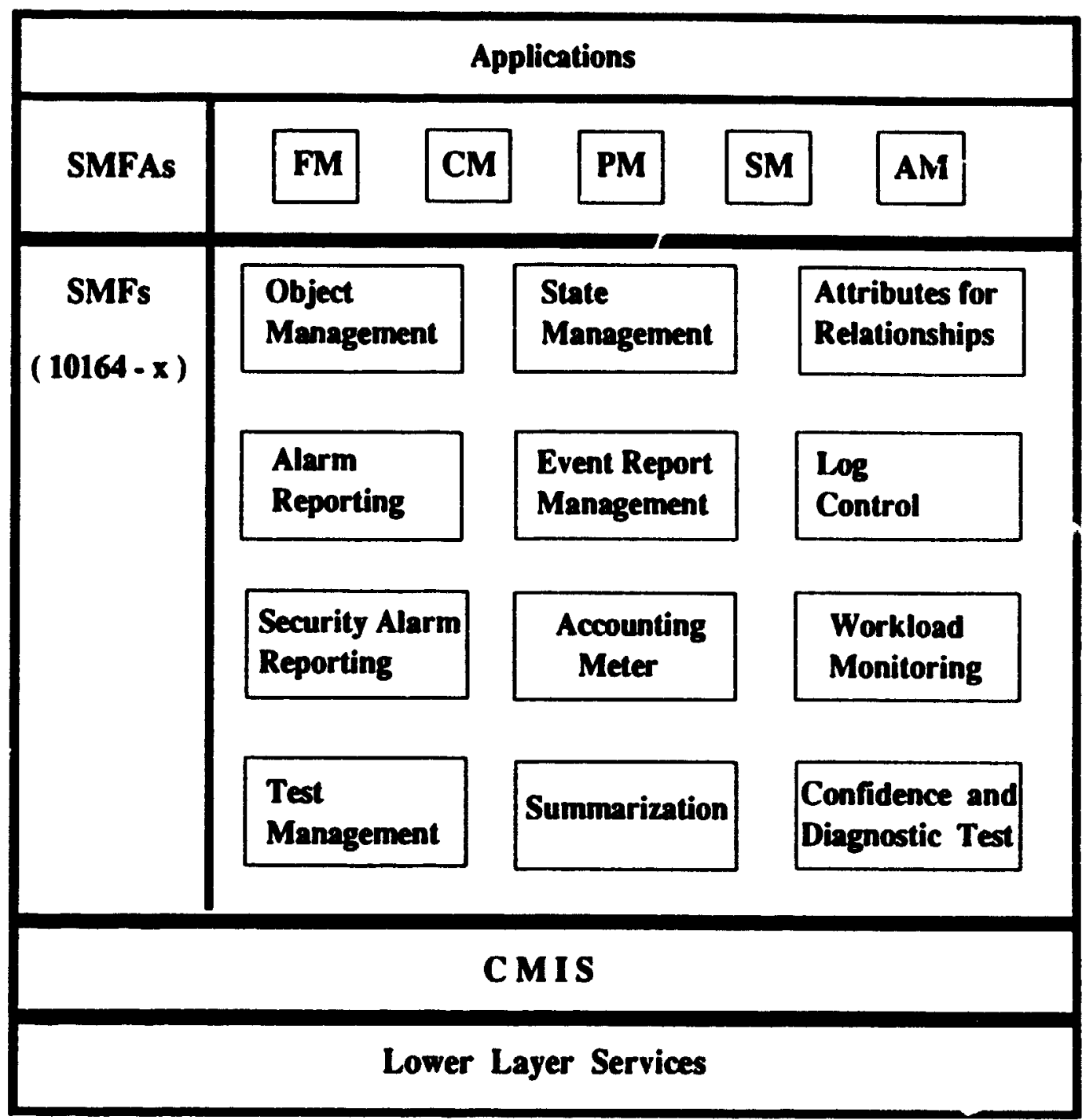

Figure 7 Relatirnship of SMFAs and SMFs (by title) 


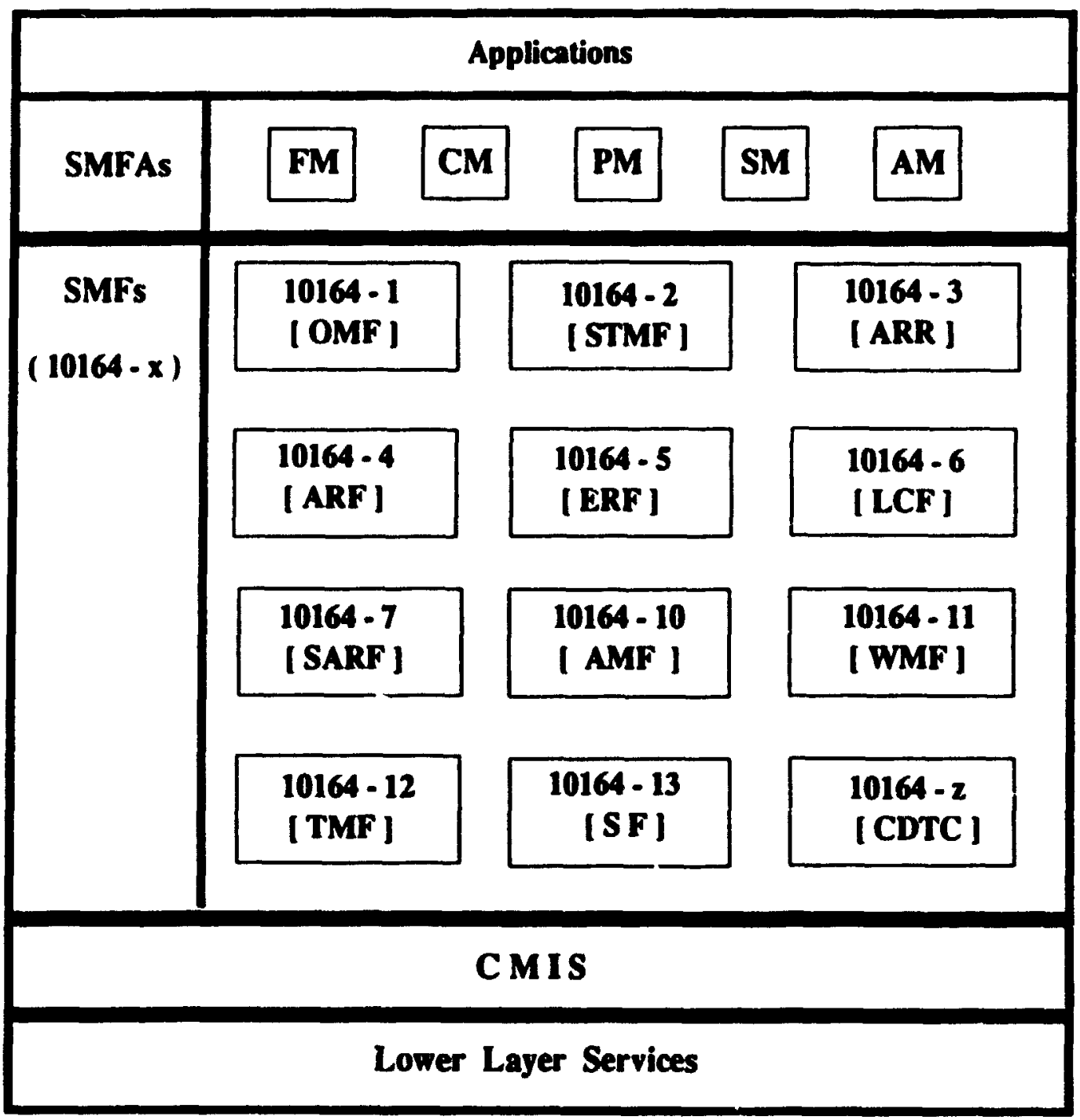

1

Figure 8 Relationship of SMFAs and SMFs (by ISO reference) 
These SMFs are only the relevant subset that provide much of the network management capabilities needed by CM and FM. Committee drafts are currently in progress for the following additional SMFs:

- Security Audit Trail [SATF] [59]:

- Accounting Metering [AMF] [56]; and

- Workload Monitoring [WMF] [57].

Working drafts are currently in progress for the following additional SMFs: Confidence and Diagnostic Testing (consisting of two documents, one specifying a Test Management Function [TMF] [58], and the other defining related management support objects classes and attributes [CDTC] [62]), and Summarization Function [SF] [61]. When additional requirements are identified, other SMFs may be developed. All of the cited documents for SMFs have been acquired for the purposes of this thesis.

Each management application can pick and choose a subset of SMFs. For instance, management capabilities encompass the functions defined in the State, Relationship. Alarm Reporting and Object Management standards, while management control includes Event Report Management and Log Control. Therefore, the SMFAs point to separate standards that contain the management functions needed to satisfy their particular requirements. For example , the Event Report Management Function may be used to satisfy FM, PM, AM and SM requirements. The Log Control function may be used to satisfy both FM and SM requirements. As well, FM makes use of Alarm Reporting and Confidence and Diagnostic Testing. 


\subsubsection{Object Management Function (OMF): DIS 10164-1 [43]}

The management user needs the ability to create, delete, examine and change the set of managed objects that forms part of the OSI environment. This document [43] defines the management function standard for these basic object management capabilities. The services are:

- Object Creating Reporting service: allows an open system to keep other open systems aware of the creation of new managed objects, so that they can address and manage these newly created objects.

- Object Deleting Reporting service: allows an open system to keep other open systems aware of the deletion of existing managed objects, because they can no longer manage the deleted objects.

- Object Name Change Reporting service: allows an open system to keep other open systems zware of name changes among existing managed objects, so that they can continue to address and manage the objects under their new names.

- Attribute Value Change Reporting service: allows an open system to keep other open systems aware of changes in attributes of managed objects.

- PT-CREATE service: requests the creation of new managed objects and the registration of their identifications, mless a specific service has been defined by one of the Systems Management Functions.

- PT-DELETE service: requests the deletion of managed objects and the deregistration of their identifications, unless a specitic ser'ice has been defiued by one of the Systems Management Functions. 
- PT-ACTION service: requests the frforming of an action on managed objects, unless a specific service has been defined by one of the Systems Management Functions.

- PT-SET service: requests the modification of attribute values of managed objects, unless a specific service has been defined by one of the Systems Management Functions.

- PT-GET service: requests the retrieval of attribute values of managed objects, unless a specific service has been defined by one of the Systems Management Functions.

- PT-EVENT-REPORT service: reports an event, unless a specific service has been defined by one of the Systems Management Functions.

\subsubsection{State Management Function (STMF): DIS 10164-2 [44]}

The management user needs the ability to examine and be notified of changes in state, to monitor overall operability and usage of resources in a consistent manner, and to control the general availability of specific resources. This document [44] defines the management function standard for managing the state of a managed object.

\section{Model}

The management state of a managed object represents the instantaneous condition of availability and operability of the associated resource from the point of view of management. Different classes of managed object have a variety of state attributes that express and control aspects of the operation of their associated resource that are peculiar to each class. However, the management state is expected to be common to a large number of resources and for this reason is standardized. Its purpose is to control the 
general availability of a resource, to make visible information about general availability, and, if a resource is not usable, to indicate what kind of action needs to be taken to make it usable. The state of a managed object does not affect its ability to be managed.

Factors Three primary factors affect the management state of a managed object with regard to the availability of its corresponding resource. These are:

- Operability: whether or no: the resource is physically installed and working, if applicable. The operability of a resource is described by the operational state attribute, which has two possible values: Enabled and Disabled.

- Usage: whether or not the resource is actively in use at a specific instant, and if so, whether or not it has spare capacity for additional users at that instant. The usage of a resource is described by the usage state attribute, which has four possible values: Idle, Active, Busy and Unknown.

- Administration: permission to use or prohibition against using the resource, impused through the management services. The administration of managed objects operates independently of the operability and usage and is described by the administrative state attribute, which has three possible values: Locked, Unlocked and Shutting Down.

\subsubsection{Attributes for Representing Relationships (ARR): DIS 10164-3 [45]}

The management user needs the ability to examine the relationships among various parts of the system, to see how the operation of one part of the system depends upon or is depended upon by other parts. Knowing the existence of one part of the system, the user needs to locate sets of other parts that are related to it. The user also needs the 
ability to change such relationships and to be notified of such changes when they occur due to other causes. This document [45] defines the general attributes, operations and notifications that can be part of any managed obiect definition.

Model A relationship is a set of rules that describe how the operation of one part of an open system affects the operation of other parts of the open system. For a relationship to be significant within the context of OSI Management, sufficient management information must be available to allow the user to identify the managed objects involved and the rules goveming their interaction. Different relationships are possible:

- A Direct relationship exists between two managed objects when some portion of the management information associated with one managed object expressly identifies the other managed object with which it has a relationship.

- An Indirect relationship exists when such a relationship can be deduced from the concatenation of two or more direct relationships.

- A Symmetric relationship exists between two managed objects when the set of generic rules governing their interactions with each other and the roles of the two managed objects are identical.

- An Asymmetric relationship exists between two managed objects when the set of generic rules governing their interactions with each other and the roles of the two managed objects differ.

Three categories of relationships are recognized by OSI Management: 
- Containment relationship is defined in Management Information Model: DIS 10165-1 [50]

- A Reciprocal relationship is a binding between two managed objects that is represented by including, as one of a set of values of an attribute of each of the managed objects, the name of the other managed object to which it is related. A managed object may have multiple instances of similar reciprocal relationships. These relationships are expressed using a set valued relationship attribute. They may be one-to-many, many-to-one, or many-!o-many without constraint.

- A One-way relationship is an asymmetric relationship between two managed objects where the relationship is expressed in the value of the relationship attribute of only one member of the pair. The relationship is represented by the type of the relationship and the name of the related managed object.

\subsubsection{Alarm Reporting Function (ARF): DIS 10164-4 [46] Faults cause open sys-} tems to fail to meet their operational objectives and they may be persistent or transient. Faults manifest themselves as particular events (e.g. errors). This document [46] satisfies the requirements for the reporting of alarms, errors and related information in a standard fashion.

Model Early detection of faults before significant effects have been felt by the user is a desisable requirement of communications systems. Degradation of service may be detected by monitoring of error rates. Threshold mechanisms on counters and gauges 
are a method of detecting such trends and providing a warning to managers when the rate becomes high.

An important criterion by which failures of communication resources are to be reported is the level to which the fault degrades the quality of service that was originally requested by (or promised to) the service user. Malfunctions will range in severity from warning, where there is no impact upon the quality of service , to critical, where it is no longer possible to provide the service requested.

Alarms are specific types of notifications conceming detected faults or abnormal conditions. Managed object definers are encouraged to include alarms information that will help with understanding the cause of the potentially abnormal situation, and other information related to side effects. An example of such diagnostic information is the current and past values of the configuration management state of the object. A single incident may cause the generation of several notifications. It is important to be able to specify in a notification some correlation with other notifications. However, the mechanism for determining the relationship between notifications resulting from a single incident is outside the scope of this function.

Alarm Type The alarm type parameter categorizes the alarm. Five basic categories of alarm are specified. These are:

- Communications alarm type: principally associated with the procedures and/or processes required to convey information from one point to another. 
- Quality of Service alarm type: principally associated with the degradation of the quality of service.

- Processing alarm type: principally associated with a software or processing fault.

- Equipment alarm type: principally associated with an equipment fault.

- Environmental alarm type: principally associated with a condition relating to an enclosure in which the equipment resides.

Probable Cause This mandatory parameter provides further qualifications as to the probable cause of the alarm. The possible set of probable causes are registered in order to allow for new values. Probable cause values for notifications shall be indicated in the behaviour clause of the managed object class definition. The managed object class definer should choose the most specific probable cause applicable. Currently, there are a total of $\mathbf{4 4}$ possible probable causes addressing the five alarm types.

\section{Perceived Severity}

There are six severity levels defined, providing an indication of how it is perceived that the capacity of the managed object has been affected. The levels defined for use with this mandatory parameter are:

- Cleared level: indicates the clearing of one or more previously reported aiarms. This alarm clears all alarms for this managed object that have the same Alarm type, Probable cause and Specific problems (if given).

- Indeterminate level: indicates that the severity level of the service affecting condition cannot be determined. 
- Critical level: indicates that a service affecting condition has occurred and an immediate corrective action is required.

- Major level: indicates that a service affecting condition has developed and an urgent corrective action is required.

- Minor level: indicates the existence of a non-service affecting fault condition and that corrective action should be taken in order to prevent a more serious fault.

- Waming level: indicates the detection of a potential or impending service affecting fault, before any significant effects have been felt.

Trend Indication This optional parameter specifies the current severity trend of the managed object. If present, it indicates that there are one or more outstanding alarms which have not been cleared, and pertain to the same managed object as that which this current alarm pertains. The Trend Indication parameter has three possible values:

- More Severe: the perceived severity in the curreltt alarm is higher (more severe) than that reported in any of the outstanding alarms.

- No Change: the perceived severity report in the current alarm is the same as the highest (most severe) of any of the outstanding alarms.

- Lesis Severe: there is at least one outstanding alarm of a severity higher (more severe) than that in the current alarm.

In order for Trend Indication to be meaningful, the perceived severity parameter value of eacii alarm that may be emitted by the managed object must be defined consistently over all of the defined alarm types for that managed object class. 
Problem Text This optional parameter allows a free form text description of the problem being reported. Understanding the semantics of this field is not required for interpretation of the alarm report. This standard does not specify the format or meaning of the data contained in the Problem text parameter. The preferred method of identifying and commiluicating additional or more specific alarm information is by registration and use of additional values for the Probable cause and/or specific problem parameters.

Problem Data This optional parameter allows the inclusion of a set of additional information in the alarm report. The information carried in this parameter may include diagnostic information and other information relating to the alarm. Problem data is a series of data structures each of which contains three items of information:

- identifier subparameter: a registered Object Identifier which defines the data type of the information subparameter. The data type must be understood by the managing system in order for the contents of the information subparameter to be parsed.

- significance subparameter: indicates whether the receiving system must be able to parse the contents of the information subparameter for the alarm report to be fully understood.

- information subparameter: carries information about the problem. This information can be parsed if the identifier is understood.

\section{Further Parameters}

The following parameters, specified by the CMIS M-EVENT-REPORT service are utilized by the alarm reporting service and included in the Alarm Record (see Figure 35). 
- Managed object class

- Managed object instance

- Event time

- Current time

Alarm Record An Alarm Record is a managed object class derived from the Event $\log$ record object class via subclassing. The Event log record object class (defined in [51]) is, in turn, derived from the Log record object class (defined in [48]). The Alarm record object class represents information stored in logs as a result of receiving an event report where the event type is one of the five alarm types described above. In addition to the attributes of the Event $\log$ record object class, the parameters discussed above, represented as attributes, are included in the Alarm record object class. The intermediate superclass, the Event $\log$ record, is defined in order to include the standard M-EVENTREPORT header parameters (see Further Parameters above). This subclassing and a partial ASN.1 ${ }^{3}$ definition for the Alarm Record is illustrated in Figure 35.

\subsubsection{Event Reporting Function (ERF): DIS 10164-5 [47]}

The management user needs the ability to modify the conditions used in the reporting of events, to select which event reports are to be sent to particular managing systems and to specify the destinations to which event reports are to be sent. This document [47] describes how a system can fulfil the basic requirement to specify conditions to be satisfied by a Potential Event Report

3 ASN.1 is discussed in Chyper 2 (2.3.5.1). 
Model

The model describes the conceptual components that provide for remote event reporting and local processing of potential event reports. The model also describes the message flows for the control messages, event reporting messages and retrieval messages. The conceptual event detection and processing function receives local notifications and forms the potential event reports. Conceptually these potential event reports are distributed to all Event Forwarding Discriminators that are contained within the local open system. The Event Forwarding Discriminator is used to determine which event reports are to be forwarded to a particular destination during specified time periods. Each Event Forwarding Discriminator contains a scheduling capability and a Discriminator Construct which specifies the characteristics a potential event report must satisfy in order to be forwarded. Event reports that have been selected are forwarded to the destination as soon as possible.

Figure 9 illustrates an example of an event report function which allows a system to establish and control the discrimination tests and the forwarding of event reports (eg. a threshold violation). At the bottom of Figure 9 is a sample discriminator construct of a test filter coded in ASN.1. 


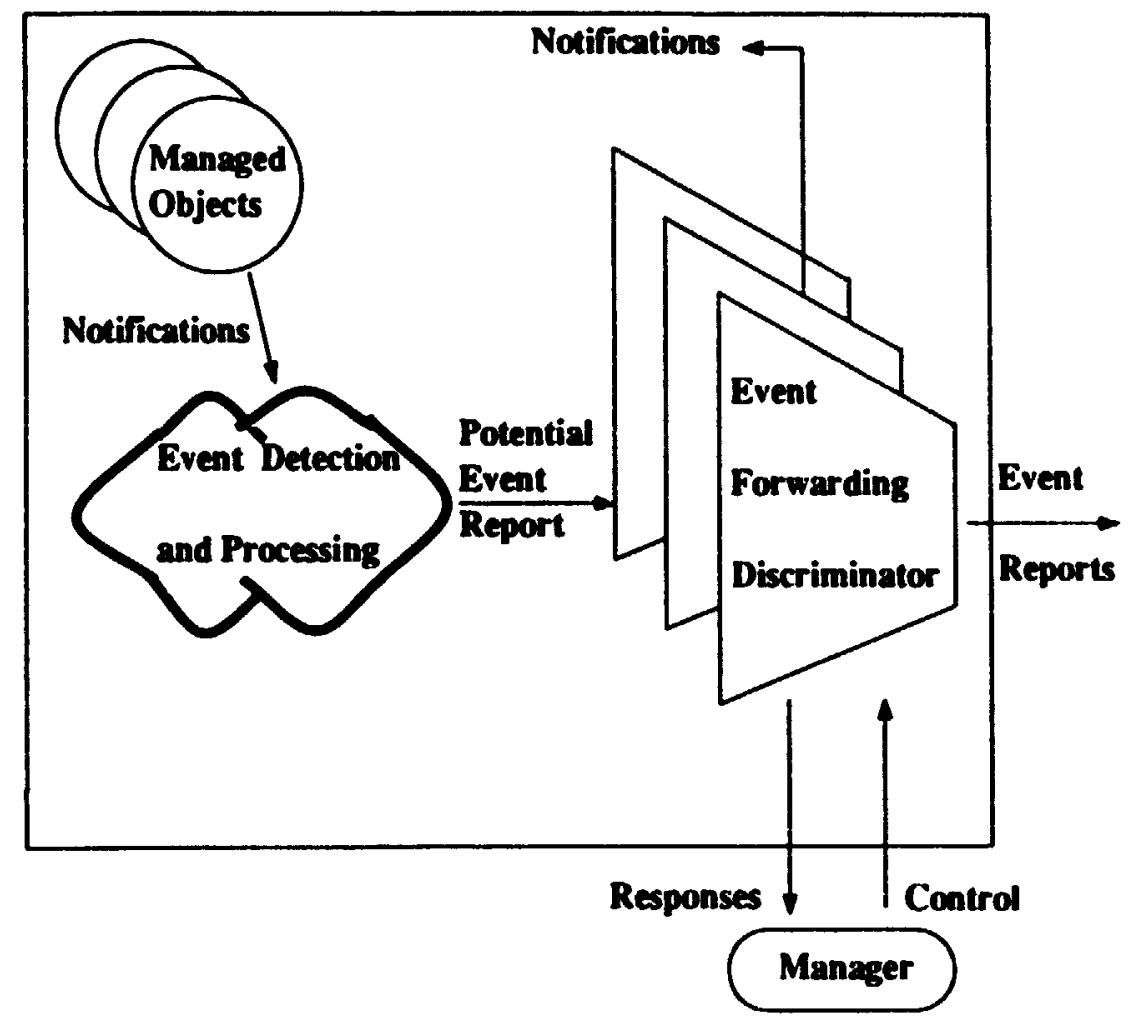

eg. Discriminator construet:

test-filter CMISFitter :: =

and ( item equality ( objectClass, Object-Class protocolEntity ), item substrings ( initialstring (entityID, PrintableString "123" )],

or [ not item equality (severity, Severity minor ], item Less0rEqual ( badPduCount, Integer 20 \}])

Figure 9 Example Event Report Function 
2.3.4.6 Ing Contrul Function (LCF): DIS 10164-6 [48] For the purpose of many management functions it is necessary to preserve information about events that may have occurred or operations that may have been performed by or on various managed objects. The $\log$ Control Function (LCF) provides services by which event reports and other PDUs can be selected and stored. Log Control provides services to initiate, terminate, suspend or resume the logging activity through the manipulation of the $\log$ object specified in [51]. Log activity can be scheruled.

A $\log$ is a management support object class that models resources as a repository for $\log$ records. A log record is a management support object class that models units of information stored in a log. The log record managed object class serves as a superclass for other record classes (eg. event log record-see Figure 35).

According to the Log Control Model [48], the $\log$ object receives event reports, or other PDUs, from various sources, and adds information ( $\log$ record identifier and logging time) to their contents to form potential $\log$ records. If the $\log$ is active, then it evaluates the potential $\log$ record according to matching criteria in its discriminator construct attribute. The contents of an event $\log$ record is determined by its notification type. The result of this sieve process yields zero, one or more log records to be stored in the log object for later retrieval[79]. Figure 10 is a schematic description of the logging capability of a system. 


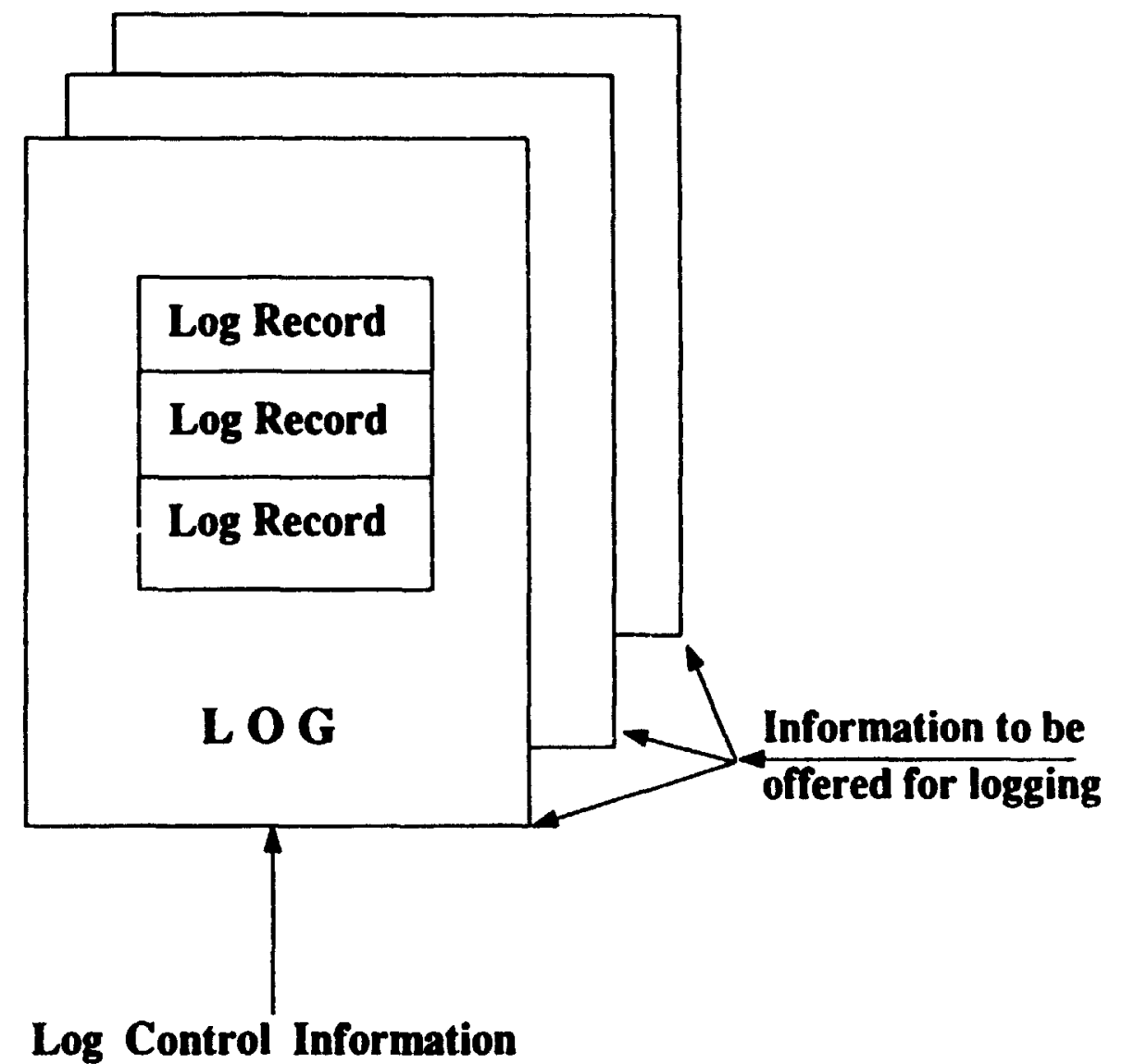

Figure 10 Log Model

\subsubsection{Test Management Function (TMF): CD 10164-12 [58]}

This document [58] is intended to satisfy requirements for the remote control of tests involving real open systems and the specification of tests which exercise OSI resources. For example, a particular test may be used to obtain a piece of information that may either 
be of use in the diagnosis of a fault or in the routine gathering of performance statistics. Requirements exist to create complex tests from simpler ones. For example, to provide the result of many subordinate tests in a single result, or to sequence tests to efficiently diagnose a fault in an entity with a large number of components. This function is seen as being applicable to different test methodologies, for example, loopback tests which configure a resource in such a way that the data sent is then received; fault injection tests in which errors are deliberately introduced in order to verify that such errors are handled properly; or self tests which simply provide a pass/fail indication.

The Test Management Function:

- defines a conceptual moúc! for the initiation, control and execution of tests and reporting of test results.

- provides the Test Results Record management support object.

- provides a Test Result notification for information reporting.

- provides a set of services to effect test management.

Environment The execution of a test involves two or more application processes. The simplest tests in:olve just two, a managing process that initiates the test, the test conductor, in one system and an agent process, the test performer, in the system containing the resources under test. The test performer is instructed to operate the test by the test conductor. Figure 11 illustrates a model of a single instance of a test invocation. 


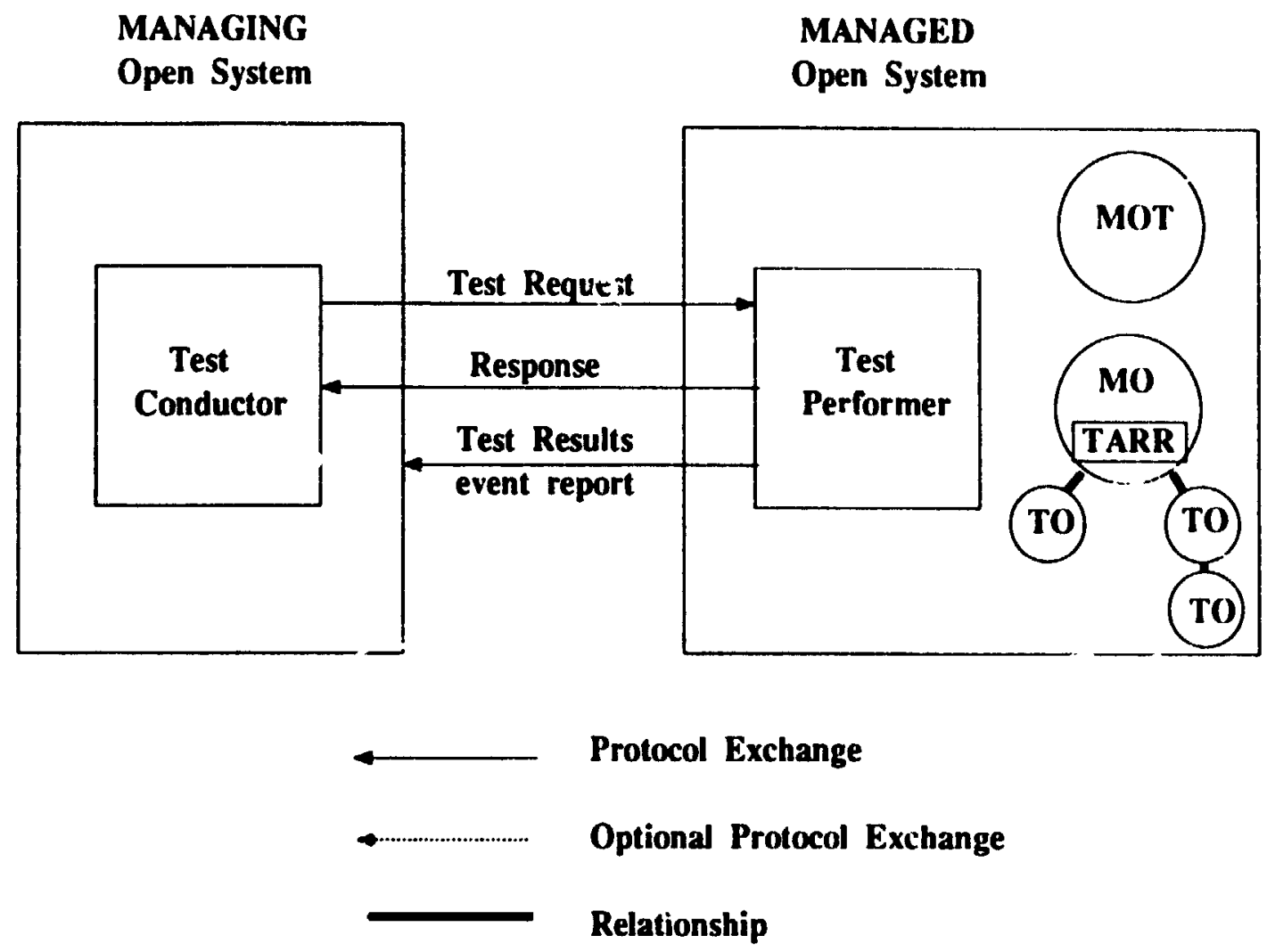

Figure 11 Test Model

A test request is addressed to a managed object being managed by the test performer and which has functionality to receive and respond to such requests. Such functionality is called the test action request receiver (TARR). Test objects (TO(s)), created by the object with TARR functionality as an effect of a test request, are required for the control and monitoring of tests and for the emission of notifications pertaining to iests. A TO is uniquely identified (and named) by a test object id (Relative Distinguished Name) and exists only for the duration of the test. Each test must involve one or more identified 
MOT, a managed object which provides management views of the subjects of tests. The execution of the test relies upon mechanisms provided by the particular resources under test, MOT(s), and TO(s).

2.3.4.8 Confidence and Diagnostic Test Classes (CDTC): 10164-z [62] This specification [62] identifies certain characteristics which are common to all classes of tests and identifies general test categories. Confidence and diagnostic test categories are required to investigate:

- the ability of a resource to perform its allotted function.

- the ability of any part of the communication system to establish a connection between a number of open systems and to transfer data without alteration between a number of open systems.

- the effec: of increased utilization of a resource.

- the ability to set up the secessary actions in order to identify the cause of a failure.

\section{Model}

Confidence anci diagnostic tests to investigate the OSI environment are described as intrusive and non-intrusive tests. An intrusive test is one by which a service disruption to the user will or may occur as a result of the test invocation. A non-intrusive test is one by which no service disruption to the user will or may occur as a result of the test invocation. Tests are defined into categories:

- Internal resource tests

- Connectivity tests 
- Data integrity tests

- Loopback tests

- Protocol integrity tests

Internal resource tests

The Internal resource test is used to specify the execution of test mechanisms which exercise the function of a resource which is internal to the system under test and to report the result of the exercise. Such test mechanisms are sometimes known as self tests. This is generally an intrusive test.

\section{Connectivity tests}

The Connectivity test is used to verify that connectivity may be established between two entities (represented by a MOT and an associated object) within a certain period of time. This test is intrusive or non-intrusive depending on the object class of the MOT.

\section{Data integrity tests}

A Data integrity test ascertains whether two entities can exchange data without any corruption, and to measure, where applicable, the time taken for the connection establishment. This is generally an non-intrusive test.

\section{Loopback tests}

The Loopback test is used to verify that data may be sent and received over a specified communications path with an acceptable error rate. The data to be sent may be specified 
either by using a simple enumeration of possibilities or by specifying a Pattern object which serves as a data generator during the test. This is an intrusive test.

\section{Protocol integrity tests}

The Protocol integrity test is to enable the control of the generation of PDUs from the MOT identified by the test. Actual responses may be compared to expected responses. It is not the function of the test to determine whether or not a particular series of PDUs indicates a fault or not. This is left to higher level functionality. This is generally an non-intrusive test.

\subsubsection{Structure of Management Information (SMI)}

To provide interoperability among network management systems, each system must have a common "view" of management information. This involves first assuring that the abstract conceptual view of management information is consistent. Within this consistent management information model, then, management information must be defined in a consistent way. Finally, a registration methodology and repository for management information definitions is required, so that general access is provided to these definitions.

Management activities require an extensive information base and a set of associated data manipulation tools. The information base is required to store information on network and system configuration, current and historic performance and trouble logs, security parameters and accounting information. This information may be viewed as a collection of MIB entries, each of which is a list of attributes and their associated values for a particular object. The following documents make the model of management information 
explicit in order to serve as a boundary between protocol and managed object designers. Note that these standards do not specify how management information is actually stored within a system.

\subsubsection{Abstract Syntax Notation One (ASN.1): ISO 8824 [35]}

In the OSI environment, the session layer (layer 5) and lower layers that provide service to it, transfer user data (i.e. uninterpreted bits conveyed between systems by some transfer mechanism) between systems. However, it is the structure and transfer of information that are of key importance to successful organizations. "Users are interested in services, not protocols."[83] Therefore the purpose of sending this data is to convey information (meanings) between the applications (services) within these systems. The latter results from the interpretation of the former within some context. The transition of data to information is the service provided by the presentation layer (layer 6) and is supported by the concepts of abstract syntax [94].

Abstract Syntax Notation One (ASN.1)[35] is used primarily to define the data structures two (or more) open systems exchange to effect a particular information processing task. The relevant information and its structure can be defined at a high level without regard for how it is represented during transit. The meaning of the information can be inferred from the knowledge of the possibilities for each of the components of the structure as defined by the shared knowledge of the ASN.1 and the Basic Encoding Rules (BER)[36]. This is analogous with the way that programmers using high-level languages, such as $\mathrm{C}++$, do not have to know in detail how shared data structures are held in computer memory or storage. 
In order to convey data values, names, operation types, etc. that relate to managed objects in the management context, a standardized representation is needed. The ASN.1 standard [35] is the notation used to express the abstract syntax of the complex data structures associated with managed object, attribute, event and action definitions to be transferred by CMIP. ASN.1 has been adopted by the ISO and CCITT as a standardized notation for describing data structures in a machine-independent form. By design, ASN.1 was meant to be machine-processable [63]. ASN.1's prime use has been in the definition of application protocols, such as CMIP.

ASN.1 is based on the formal theory of abstract data types as developed in [22] and is defined using the Backus-Naur Form (BNF) notation [94]. ASN.1 has no notation for "defining operations and the behaviour of operations", [4]. On the other hand, the main benefit of ASN.1 and its BER is its coding of values aspect.

\section{The Basic Encoding Rules (BER)}

The BER generate encodings in a tagged data format. The encoding of every data value in an abstract syntax, whether an entire Protocol data unit or some component of it, is transferred as a triple of $<$ Type, Length, Value $>$. The Type identifies what type of datum is encoded. A type is a (non-empty) set of values, and represents a potential for conveying information. A type may be simple or structured. More complex data types are built recursively from simple types (e.g. to create a record, a structure type is used whose data filed contains ASN.1 objects corresponding to the fields in the record). The Length is the length of the value section. The Value is the actual value itself, in a well-defined bit order [94]. 
The three parts are actually termed Identifier (I), Length (L) and Contents (C). The Identifier field conveys three pieces of information; the tag class of the data value being conveyed; the tag number, the form of the encoding - whether it is primitive or constructed. The Length field has three variants: (short, long: (definite)), and (indefinite). The Length (together with the form) allows the end of the contents to be found. The receiving system need not understand the tag to find the end of the contents, and this allows an encoding to be skipped if it cannot (yet) be decoded. The Contents field is the substance of the encoding, conveying the actual value. When the form of the encoding is primitive, the contents is simply a series of octets (zero or more). When the form is constructed, the contents is a series of nested encodings, each itself having Identifier, Length and Contents. This nesting can be as deep or shallow as needed; its primary purpose is to convey values which have components which themselves have components, and so on, to any depth.

\section{ASN.1 Primitive types}

The primitive types of ASN.I are:

- INTEGER;

- BOOLEAN;

- BIT STRING; IA5String, GeneralString, GraphicString, GeneralizedTime, NumericString, PrintableString, T61String, TeletexString, UTCTime, VideotexString, Visiblestring, OCTET STRING. 
Some examples of ASN.1 primitive types are:

- generalized time type (GeneralizedTime), with the precision of the time representation indicating the granularity of the time measurement (i.e. YYYYMMDDHHMMSS.SSS and an indication of the time differential (HHMM) between local and universal time can be appended).

- For example, the string 19890613123012.333-0500 represents a local time of 12:30:12 (and 333 msecs) on the 13th June 1989, in a time zone which is 5 hours behind GMT.

- universal time type (UTCTime), is intended for use in intemational applications where the local time alone is not adequate, and the flexibility to use all the forms allowed by GeneralizedTime is not required (i.e. YYMMDDHHMM and an indication of the time differential (HHMM) between local and universal time can be appended).

- For example, the string $8906131231-0500$ is the UTCTime representation of the previous GeneralizedTime example.

\section{ASN.1 Composite types}

The following are ASN.1 composite types:

- CHOICE - where values are chosen from those of a number of component types;

- SEQUENCE/SET - where values are collections of component values of different types;

- OPTIONAL;

- Integer Constants. 
The ASN.1 "CHOICE" is where values are chosen from those of a number of component types. The ASN.1 "SEQUENCE/SET" is where values are collections of component values of different types. The ASN.1 "SEQUENCE OF/SET OF" is where values are collections of component values of a single type.

The following example ASN.I composite types:

- sequence type (SEQUENCE), is intended for use where, for example, tide mark is a composite of two integer types and one of generalized time.

- TideMark ::= SEQUENCE

currentValue INTEGER (0..4294967295),

previousValue DNTEGER (0..4294967295),

lastResetTime GeneralizedTime

- choice type (CHOICE) is intended for use where, for example, object class is a choice of either an object identifier type or an integer type.

- ObjectClass ::= CHOICE IglobalForm [0] OBJECT IDENTIFIER, localForm [1] INTEGER ।

\section{object identifier type}

An object identifier is a data type denoting an authoratively named object, regardless of the semantics associated with the object. The values of the object identifier type [94] correspond to the nodes of the object identifier tree (OIT), a tree set up to allow the unambiguous naming of information objects. The tree consists of a root connected to a number of nodes via edges. Each label consists of a non-negative integer value and 
possibly a brief textual description. Each node may, in tum, have children nodes of its own, termed subordinates, which are also labelled. Central to the notion of the object identifier is the understanding that administrative control of the meanings assigned to the nodes may be delegated as one traverses the tree. Every organization who wishes to allocate object identifiers (i.e. every organization who wishes to design or register one or more information objects) requests a node in the tree. Particular designs are placed at the leaves of this tree, subordinate to the organization which is registering them. Each node of the tree provides unique (non-negative) integers for each of its subordinates. It is then possible to uniquely identify any node as a sequence of integers. A subset of the upper reaches of the OIT, extending from the root, are shown in Figure 12. It can be seen that the $\{0\}$ node is administered by CCITT, the $[1]$ node by ISO and the 2$\}$ jointly by CCITT and ISO. From this example, ISO/EC managed object identifiers will

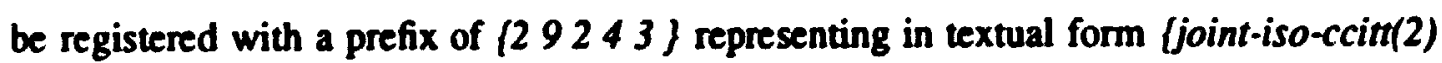
management(9) function(2) $10164-4(4) M O C(3))$. Figure 30 is another example of the object identifier registration, but at the leaf end of the ASN.I object identifier tree. 
RFCI156-mib OBJECT IDENTIFIER ::= liso org(3) dod(6) internet(1) mgmt(2) 1$\}$ ARF-MOC OBJECT IDENTIFIER $\left.\quad:==\begin{array}{llllll}2 & 9 & 2 & 4 & 3 & 1\end{array}\right)::=2.9 .2 .4 .3 .1$

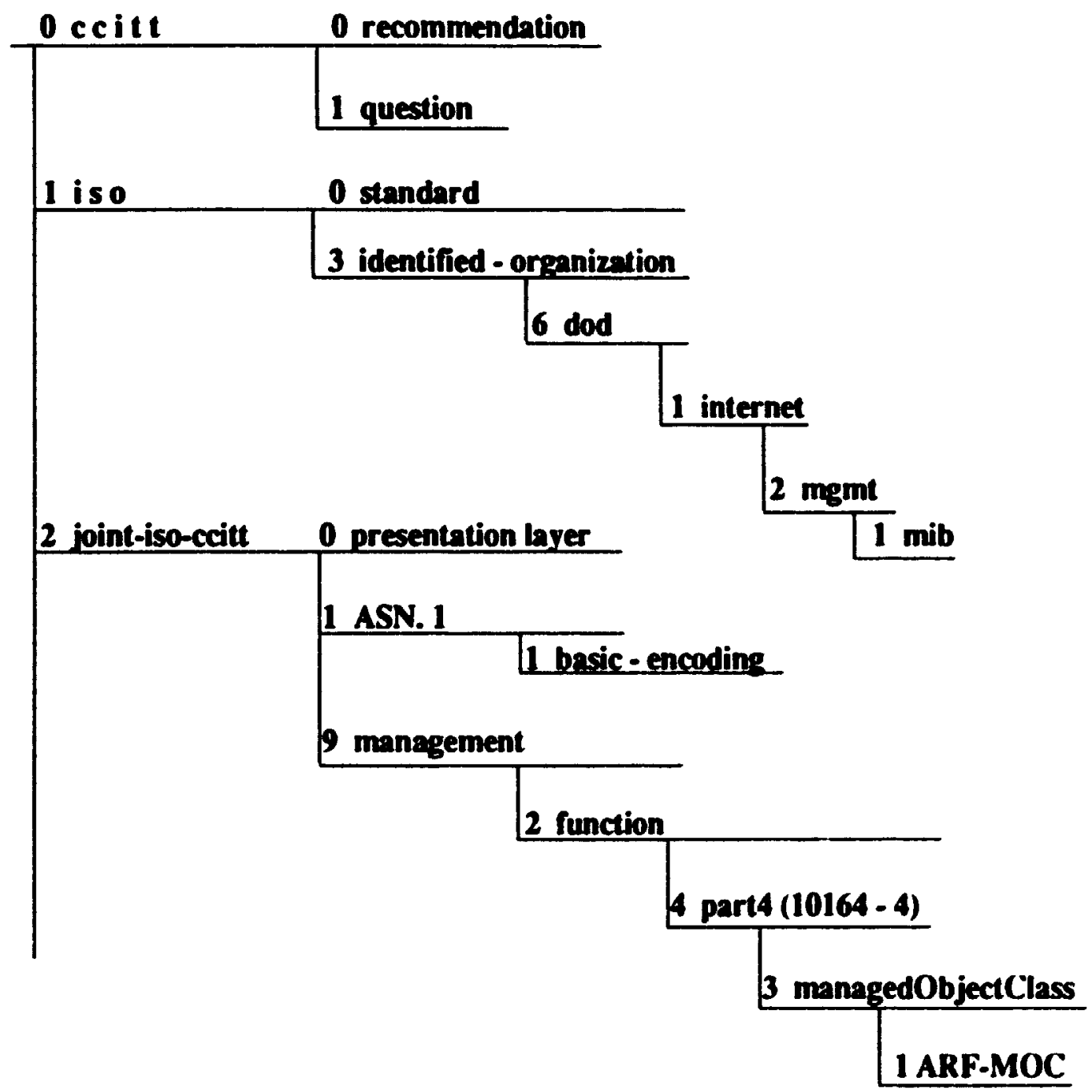

Figure 12 Example of ASN.1 Object Identifier Tree 
This document [50] defines the Information Model of managed objects and their attributes relating to the systems management model introduced in the SMO [42]. It also defines the principles of naming managed objects and attributes so that they may be identified in and accessed by management protocols. "In the Information model, object-oriented design is applied to the specification of information conveyed between open systems in systems management protocols. It need not be applied to system implementation," [50].

\section{Classes and Instances}

Instances of managed objects that share the same type of properties are said to be of the same managed object class. Extensions made to one class by defining new properties in order to define a new managed object class is called specialization.

Object-oriented programs are organized around classes that reflect the domain they are about. During execution of a program, instances of these classes are created, initialized and then manipulated. Classes must be defined before instances are created. A class definition specifies instance structure. In $\mathrm{C}++$, instances are called objects of a class and attributes are called member data elements.

\section{Encapsulation}

Another facet of object-oriented design is the concept of encapsulation. Encapsulation ensures that the integrity of an object is preserved. How operations are performed and how the appropriate consistency constraints are enforced is determined by the definition of the managed object class. 
In object-oriented programming languages, the structure of an object is intended to be opaque outside of certain restricted scopes. Access to the structure is provided by operations associated with the class of the object. C++ has many different loopholes to break encapsulation, however the preferred style for public access is through userdefined virtual member functuons.

Model The model for management information includes managed objects, system management operations and notifications, inheritance and allomorphism, containment and a naming tree [50]. It fot,ns the basis from which an understanding of the nature of managed objects may be obtained.

System Management Operations System management operations are operations on a managed object to effect systems management. Two kinds of operations are defined: those which can be sent to a managed object to be applied to its attributes, and those which apply to the managed object as a whole. The constraints on the performance of an operation on a managed object is defined in its class definition. The following operations can be sent to a managed object to be applied tu its contained attributes: Get attribute value, Replace attribute value, Set-to-default value, Add member and Remove member.

In object-oriented programming languages, operations are composed of independently defined implementations specific to particular classes. In $\mathrm{C}++$, these are called virtual member functions. When an operation is invoked, a runtime dispatch selects the appropriate implementation. In $\mathrm{Ct+}$, the terminology for invoking an operation is calling a member function. The C++ syntax emphasizes the selection of the function based on 
the object (e.g. to invoke a function GET on an instance "MO", C++ uses: MO.GET (attribute_ids) ).

\section{Notifications}

Notifications are emitted by managed objects when some internal or external event occurs. Notifications are specific to managed objects that emit them. The notifications, and the information they contain, are part of the definition of the managed object class of which the managed object is an instance. Whether or not notifications are transmitted externally as protocol, or logged, depends on the management configuration of the open system.

\section{Inheritance}

Inheritance is the conceptual mechanism by which attributes, notifications, operations and behaviour are acquired by a subclass from its superclass. This is also known as an "is-a" relation. Multiple inheritance allows a subclass to acquire elements from more than one superclass. Each managed object class defines the superclass(es) from which it has been derived. The inheritance hierarchy is the arrangement of managed object classes where the hierarchy is organized on the basis of the class specialization. A subclass is lower in this hierarchy than its superclass(es). Inheritance is shown in Figure 29 and Figure 35. One object class, called "TOP" (root), is designated as the ultimate superclass in this class hierarchy. The ASN.1 definition of TOP is shown in Figures 13 and 14. 
Object-oriented programming languages support the specification of a new class as an incremental modification of other previously defined classes. A new class is said to inherit from these other classes, which means that its effective definition is a combination of what is explicit in its own definition and what is in those classes that from which it inherits. In $\mathrm{C}++$, the terminology used is that of a new derived class is defined in terms of a previously existing base class.

Some of the most complex issues having to do with inheritance in $\mathrm{C}++$ only arise when multiple inheritance is allowed (i.e. when a new class can be made to inherit from more than one superclass). C++ supports the use of multiple inheritance, however users are forced to explicitly resolve clashes from multiple base classes.

Allomorphism ("allo" $\rightarrow$ "other") Allomorphism is the ability of an instance of a subclass to resemble the behaviour of its (previous version) superclass as observed by systems management protocols. Allomorphism permits an enhanced managed object class definition to be extended in a way which permits interoperability through "backward compatibility" when either one manager or one managed object of the management information exchange does not include the relevant enhancement (i.e. a previous version). This capability is required so that migration between versions of management is possible. Managed objects that support allomorphic behaviour are required to contain a set-valued attribute that identifies the set of superclasses which it is capable of imitating.

C++ does not support allomorphism. 


\section{Containment}

Containment is the relationship whereby one class can contain other managed objects of the same or different classes. The containing managed object is known as the superior managed object and the contained managed object is known as the subordinate managed object. A subordinate managed object may be contained in one and only one superior managed object. This is also known as a "part-of " relation. Containing managed objects may themselves be subordinate managed objects. This hierarchy can be visualized as a directed graph with each edge pointing from a subordinate to a superior. Superior managed objects may themselves be recursively contained in other managed objects. The containment hierarchy can be used to model real world hierarchies of parts or real world organizational hierarchies. Figure 28 and Figure 34 are examples of containment.

\section{Naming Tree}

The naming tree can be visualized as a single-rooted hierarchy which represents the naming structure of managed objects. The containment relationship is used for naming managed objects. Names are designed to be unambiguous in a specified context and this context is determined by the containing object. Thus superior managed objects become the naming contexts and their names brcome the names of the contexts. The hierarchy can be visualized as a directed graph with each edge pointing from a named managed (ibject to a naming context. Note that the naming tree does not necessarily represent the physical containment. The top level (TOP) of the naming hierarchy is referred to as the root which is a null object that always exists. A managed object can exist only if its superiour managed object exists and therefore every managed object has a name. 
A managed object class is externally identified by an ASN.1 object ide tifier. The object identifier can be viewed as a sequence of integers that navigate through the registration tree to the managed object class (see Figures 12 and 30). Note that the registration tree is not related to either the containment tree or the naming tree.

Except for the class root, each managed object class must include at least one attribute suitable for naming. A suitable attribute is one that can be tested for equality and whose semantics permit its value to be fixed for the lifetime of each managed object that uses it for naming. This value should uniquely identify the managed object within its naming context. Thus, an attribute value assertion (AVA) about this attribure uniquely identifies a managed object within the scope of its containing managed object. When used for this purpose, the attribute value is called a relative distinguished name (RDN). The attribute "Name", inherited from TOP, is included in every managed object class for naming purposes. No operations other than Get (i.e. read only) are permitted on the Name attribute (see Figure 13).

The concatenation, in a sequence, of the relative distinguished names of its superiours in the naming tree, starting at the root down to the intended managed object, serves to uniquely identify that managed object. This sequence that distinguishes one entry frum another is called the distinguished name (DN) of the managed object.

Naming Example The naming of a managed object class is defined by means of the Name Binding template. This template identifies the managed object class being named and defines the relative distinguished name that will be used to name instances of the class in the context of a particular superior class. This template also provides for the 
specification of relationships that exist between two object classes as a consequence of a particular name binding. Figure 27 represents the IEEE LAN Bridge Name Bindings. There are two issues here. First, there's the distinction between classes and instances (specific objects). The set of objects at any time is constrained to be a naming tree. Thus each object other than "root" has exactly one superior (eg. "is part-of" one superior). However, a class can have more than one superior (i.e. can participate in more than one NAME BINDING template in the SUBORDINATE OBJECT CLASS role). For example:

binding-1 NAME BINDING

SUBORDINATE OBJECT CLASS class1;

NAMED BY SUPERIOR OBJECT CLASS class2;

WITH ATTRIBUTE attri;

REGISTERED AS ( oid । ;

binding-2 NAME BINDING

SUBORDINATE OBJECT CLASS class1;

NAMED BY SUPERIOR OBJECT CLASS class3;

WITH ATTRIBUTE attr2;

REGISTERED AS \{ oid2 \};

This says that an instance of class class 1 can be the child of either a class 2 or a class 3 object. Thus it would be possible to have two objects $\mathrm{O} 1$ and $\mathrm{O}$, both of class class1, where $01 \mathrm{~s}$ parent was a class 2 object and $02 \mathrm{~s}$ parent was a class3 object. The 
name attribute of 01 would have the form $\mid$ attr $l=x x x \mid$, while 02 s name attribute would have the form $\{$ attr $2=y y y\}$.

The second issue is that the nameBindings attribute has to do with subordinates not superiors. Any object can (and, in general does) have multiple children (subordinates) in the containment hierarchy (i.e. "part-of" relation). The nameBindings attribute is supposed to be the set of all name bindings that describe children of a given object. For example, if $\mathrm{O} 3$ is an instance of class 3 , then $\mathrm{O} 3$ might have children of class class 1 , in which case oid 2 would be among the members of its nameBindings attribute. To summarize, the name attribute describes the edge from a given object to its parent in the "part-of" relation, while the nameBindings attribute summarizes information about the object's children

\section{Attributes}

Each individual attribute of a managed object is defined by an ASN.1 object identifier. An attributes's object identifier distinguishes it from all other attributes of the managed object. For instance, Figures 25 and 26 illustrate the managed objects and their attributes for the IEEE LAN Bridge. The right side of Figure 30 illustrates the OSI registration subtree, ieeeBridgeAttributes, containing the unique OBJECT IDENTIFIER nodes for the 54 attributes of the IEEE LAN Bridge.

It is necessary to provide tools to explore the managed objects contained in a system. Therefore, the following attributes are defined for all managed objects: Name, Object Class, Allomorphics, Name Bindings and Packages. These mandatory attributes can be 
thought of as shared management knowledge which is defined in the managed object class TOP by DMI [51]. This public domain knowledge provides the ability to determine which managed objects have been instantiated in a given system. Note that no information is gained by this method concerning the capabilities of a system with respect to managed object classes which have no managed objects instantiated within the system. In other words, potential support or non-support within a system of non-existent managed objects cannot be inferred.

\subsubsection{Definition of Management Information (DMI): DIS 10165-2 [51]}

This standard [51] defines generic managed objects classes, attributes types, notifications types, actions types and parameter types. This includes management support objects and generic superclasses, but does not include layer objects. DMI also specifies compliance requirements placed on other standards that make use of these definitions.

The source of some of these definitions is the SMF standards; therefore they represent information types that are required in order to perform particular systems management functions. As such the set of definitions contained within this document is open-ended as more management support objects or generic subc asses may be required. For instance, as of June 1990. the summarization support objects are defined in SF [61] "until the appropriate portions can be moved to DMI".

Attribute types and specific attributes are defined in this document using Guidelines for the Definition of Managed Objects (GDMO) [52] (see 2.3.5.5). The following is the current list of managed object classes defined in the standard: Alarm Record, 
Attribute Value Change Record, Discriminator, Event Foru'arding Discriminator, El'ent Log Record, Log, Log Record, Object Creation Record, Object Deletion Record, Object Name Change Recond, Relationship Change Record, State Change Record, Security Alarm Report Record, System and TOP.

These definitions may be used to define management information in other standards, either directly or by specialization. The means by which such references are made are described in Guidelines for the Definition of Managed Objects [52]. When DMI contains a suitable definition of management information, it is recommended that this definition is referenced rather than defining a new information element with the same structure.

top MANAGED OBJECT CLASS

CHARACTERIZED BY

topPackage PACKAGE

BEHAVIOUR DEFINITIONS topBehaviour;

ATTRIBUTES

objectClass GET,

name GET,

allomorphs GET,

nameBindings GET,

packages GET:::

REGISTERED AS \{objects 1);

Figure 13 ASN.1 of TOP Managed Object Class 
Top-Syntax (iso(1) org(3) dod(6) internet(1) $\mathrm{mgmt}(1) \operatorname{mib}(1)$ $\operatorname{oim}(9) \operatorname{misc}(4) 21$

DEFINITIONS ::=

BEGIN

- from ISO/IEC DIS 10165-2: Definition of Management Information Allomorphs ::= SET OF OBJECT IDENTIFIER

NameBindings ::= SET OF OBJECT IDENTIFIER

Packages ::= SET OF OBJECT IDENTIFIER

- From Directory InformationFramework

RelativeDistinguishedName ::= SET OF Attribute ValueAssertion

AttributeValueAssertion ::= SEQUENCE (AttributeType,

AttributeValue)

AltributeType ::= OBJECT IDENTIFIER

AttributeValue $::=$ ANY

- From CMIP-1

ObjectClass ::= CHOICE [globalForm [0] OBJECT IDENTIFIER, localForm [1] INTEGER । END

Figure 14 ASN.1 of TOP Managed Object Class (cont.) 
It should be noted that all managed object classes are ultimately derived, by specialization, from the managed object class TOP. Therefore it is necessary to reference the definition of TOP even if no other information defined by DMI is found to be useful. Figures 13 and 14 are the ASN.1 representations of TOP as defined in [67].

2.3.5.4 10165-3: Definition of Management Attributes [40] This standard no longer exists; the material was incorporated into 10165-2 [51].

\subsubsection{Guidelines for the Definition of Managed Objects ((IDMO): DIS 10165-4} [52]

Two network management implementations can interoperate only if there is a common (public domain) managed object supported on both sides of the interchange. This document [52] allows different organizations, including private companies, etc., to define their own managed objects in the required consistent way. This document recommends notational tools to be used in such definitions, and what documentation structure is to be used for managed object class definitions in order to ensure compatibility with OSI management standards. Included in GDMO are templates for management information definitions. These templates provide common, detailed descriptions for defining managed object classes, name bindings, attributes, actions and notifications. Specification techniques and guidelines for basic object management capabilities should reduce duplication of effor by identifying commonly useful documentation layouts. procedures and definitions. 
The process of defining managed object classes requires the registration of globally unique identifiers, known as Object Identifiers, that correspond to various aspects of the managed object class, such as the object class name, attribute types, etc. ASN.1 [35] specifies the structure of the object identifier and the values of the initial arcs (see Figure 12). Further information on the establishment of registration mechanisms and registration authorities may be found in DIS 9834 [53]. This area was outside the scope of this thesis research.

Notational Tools for Managed Object Definition This section provides descriptions of the notational tools used to define the templates defined in [52]. For a complete description of the notational tools specified for OSI management, see [52].

Overview of Notational Tools The "Templates" define a common set of tools and a common format for the representation of the various aspects of a managed object class definition and its associated naming structure. Examples of the use of these tools may be found in Annex $A$ of [52] and is shown in Figures 13 and 14 above.

The structure and behaviour of a managed object class is primarily defined by means of the Managed Object Class Template. This template identifies the inheritance relationships that exist between the class and other managed object classes, the packages of class specific behaviour, the attributes that are associated with the class, the notifications that the class may issue and the operations that may be performed upon the class. In order to allow re-use of parts of this specification in the specification of other managed object classes. additional templates are defined to provide for the specification of attributes (in- 
dividual and group), behaviour, actions, notifications and conditional packages. These other templates are "called up" by the Managed Object Class template: this mechanism allows references to be made in one standard to specifications that are contained in other standards, hence allowing "generic" specifications to be made available for use in managed object class definitions in addition to local specifications. Templates may also be included "in-line" if so desired.

The naming of a managed object class is defined by means of the Name Binding template. This template identifies the managed object class being named and defines the relative distinguished name that will be used to name instances of the class in the context of a particular superior class. This template also provides for the specification of relationships that exist between two object classes as a consequence of a particular name binding.

Template labels shall be unique within the standard or document in which they are declared. Where a template-label is declared in docum,ent $A$ and referenced in document B, the reference in document B shall be prefixed by the globally unique name of dxcument A. In the case of documents named by an internationally recognized naming authority such as [CCITTISO/IEC], the registered name of the document shall be used as the identifier, such as [Recommendation X.7221ISO/EC 10165-4]. Where a globally unique name is not already available, it is permissible to assign the value of an OBJECT IDENTIFIER. to the referenced document, and use this value as a globally unique document name.

Label references that are not prefixed by a document-identifier are assumed to refer to labels declared in the document in which the reference appears. Whenever a template- 
label is present in a template as pointer to another template, it may be replaced by the entire text of the referenced template itself (including the template's label).

Managed Object Class Template The Managed Object Class template forms the basis of the formal definition of a managed object. Elements in the template allow the class to be placed at the appropriate node of the inheritance tree, the various attributes of the class to be specified, and the behaviour of the class to be defined. The major elements of the definition are:

- Inheritance. Each managed object class defines the superclass(es) from which it has been derived. All characteristics of the superclass(es) are inherited by the subclass; the subclass definition may add to these characteristics (refinement) but may not remove any characteristics of the superclass. Ultimately, all classes are subclasses of TOP;

- Allomorphs. If the class supports allomorphism, the set of classes that are legitimate allomorphic views of the class must be defined. These classes must all be superclasses of the class being defined:

- Mandatory Packages. The managed object class definition includes the packages of behaviour, attributes, operations and notifications that provide a complete specification of the behaviour that is common to all instances of the class;

- Conditional Packages. The managed object class definition includes the specification of packages of behaviour, attributes, operations and notifications that are present or absent in instances of that class as a consequence of a specified condition; and 
- Class Naming. The managed object class definition must include a class name which may be used to refer to the class in CMIP. This is achieved by registration of an Object Identifier value which corresponds to the class.

DERIVED FROM The DERIVED FROM clause shall be present in all managed object class definitions other than "TOP". It is therefore the case that "TOP" is a superclass of all classes other than itself.

The class-label identifies a managed object class from which the managed object class has been derived; i.e., a managed object class which is one of the object class's parents in the inheritance hierarchy. As multiple inheritance is permitted, a managed object class may have one or more parent classes.

The process of inheritance (specialization) requires all the characteristics of the superclass(es) other than DERIVED FROM and ALLOMORPHIC SET to be included in the definition of the subclass. If it is intended that the subclass be allomorphic, the definition of the subslass shall include a ALLOMORPHIC SET clause that explicitly defines the set of classes that the subclass is aliomorphic to.

Where multiple inheritance results in the same element definition being multiply imported (as could happen, for example, if two parent superclasses contain the same attribute), the subclass is assumed to contain a single copy of the definition concerned.

Characteristics that are inherited from a superclass shall not be repeated in the documentation of the subclass unless one of the techniques described in ISO/IEC 10165-4 for extending or modifying an element of the superclass is being used. The DERIVED 
FROM clause is therefore presumed to automatically import all inheritable elements of definition from the superclass definition(s).

AJLOMORPHIC SET The Allomorphic Set allows a set of superclasses to be identified that are "backwards compatible" with the managed object class. Thus, if managed ubject class $A$ identifies classes $B$ and $C$ as members of its allomorphic set, it is possible (1) uperate on an instance of class $\mathrm{A}$ as if it were an instance of class $\mathrm{B}$ or $\mathrm{C}$. The definition of allomorphic forms allows, for example, the definition of enhanced versions of a managed ubject class that are backwards compatible with previous versions. The class lahel shall identify the class-label of a managed object class definition that is a superclass of the managed object class that is being defined.

\section{BEIHAVIOUR}

OSI standards do not include a formal framework for defining the behaviour of managed objects [3]. Therefore, only a tex:uai description of what a network resource managed object class represents, including its function has been adopted:

- a description of the relationships that sccur tetween different instances of the man:aged ubjecl class being defined, as well as those that can occur between instances of the managed (object class being defined and instances of other managed object classes.

- a description of the operations that are supported by the managed object class, with precise definition of the effects, side effects if any, consuraints, response notifications, failure modes. 
- specification of how instances of this managed ubject class are created and deleted. particularly whether they can be created/deleted via management Create/Delete uperations.

- a description of the notifications that can be generated, the conditions that generate them (e.g. crossing of a threshold), their contents and side effects. if any. In particular, identify all the attributes that are subject to the AC and SC notifications, if the not. are supported.

- other constraints, including those involving other managed object classes.

This lack of a formal definition of behaviour permits inconsistencies in both interpretation and implementation. The role of network managers and network diagnosticians will be difficult without rigorous, certified conformance and interoperability testing of managed objects' behaviour.

Initial Value Managed Cinjects Initial Value Managed Objects (IVMO) are most useful in cases where managed object classes that do not support the Create operation have been defined. An IVMO provides a mechanism that allows initial values of all attributes (or a subset) of a managed object class to be controlled by management. IVMOs represent the collection of characteristic attributes that supply default and initially advertised attribute values to be used by instances of the managed object class when the instances are created [78]. An IVMO is address $2^{2} \cdot$ le and appears in the MIB. A managed object can be queried for its IVMO. 
In $\mathrm{C}++$, buth the name and type of member data elements must be specified in the class definition. Default initialization expressions can be supplied for each member data element. Objects of a class are created by calling an automatically defined constructor function for the class. C++ has mechanisms for defining initial value arguments for the constructor function; if no value is provided to the constructor function, the default initialization expression in the class definition is used to determine the initial value for a new instance's member data element.

June 1991 IS()/IEC Developments Appendix B lists the change in statis of the ISO Network Management standards as a result of ISO meeting in Arles, France in June 1991. 


\section{CHAPTER 3 DARPA Management Standards}

\subsection{The Organization}

In 1968. The United States Defense Advanced Research Projects Agency (DARPA) initiated an effort to develop a technology which is known as packet switching. This technology had its roots in message switching methods, but was strongly influenced by the development of low cost minicomputers and digital telecommuniations techniques during the mid-1960's. During the 1970`s, DARPA initiated a number of programs 11 explore the use of packet switching methods in alternative media including mobile radiu. satellite and cable. The successful implementation of packet radio and packet satellite technology raised the question of interconnecting ARPANET with other types of packet nets. The solution was in the form of an intemetwork protocol and a set of gateways io connect the different networks. This solution was further developed as part of a research program in internetting sponsored by DARPA and resulted in a collection of computer communications protocols based on the original Transmission Control Protecol (TCP) and its lower level counterpart, Internet Protocol (IP). Together, these protocols, alıng with many others developed during the course of the research, are referred to as the TCP/IP Protocol Suite. TCP/IP provides definitions of connectivity functions for buth local and wide area networks. A standardized addressing procedure is used for the major TCP/IP networks to insure uniqueness of addresses, thus permitting connectivity between enterprises. This collection of TCP/IP interconnected networks is known as the 
Internet. Given the proper authority, a user on any of these standard TCP/IP networks can communicate to users on any of the other TCP/P networks.

In the early stages of the Internet research program, only a few researchers worked II develop and test versions of the internet protocols. Over time, the size of this activity increased until, in 1979, it was necessary to form an informal committee to guide the technical evolution of the protocol suite. This group was called the Internet Configuration Control Board (ICCB). In January, 1983, the Defense Communications Agency, then responsible fo- the operation of the ARPANET, declared the TCP/IP protocol suite to be standard for the ARPANET. Later that year, the ICCB was reorganized around a series of task forces considering different technical aspects of internetting. The re-organized group was named the Internet Activities Board.

The Internet Activities Board (IAB) is the coordinating committee for Internet design, engineering and management [15]. IAB members are committed to making the Internet function effectively and to evolve to meet a large scale, high speed future. The IAB focuses on the TCP/IP protocol suite, and extensions to the Internet system to support multiple protocol suites. The IAB sets Internet Standards, manages the Request for Comments (RFC) publication process, performs strategic planning for the Internet, acts as an international technical policy liaison and representative for the Internet. All decisions of the IAB are made public. The principle vehic's by which IAB decisions are propagated to interested parties are the Internet Monthly Report and the Request for Comments (RFC) memo series.

The Request for Comments documents (RFCs) are working notes of the Internet 
research and development community. A document in this series may the essentially any topic related to computer communication, and may be anything from a meeting repurt in the specification of a standard. Most RFCs are the description of network protocols or services, often giving detailed procedures and formats providing the information necessary for creating implementations. Other RFCs report on the results of policy studies or summarize the work of technical committees or workshops.

While RFCs are not refereed publications, they do receive technical review from either task forces, individual technical experts, or the RFC Editur, as appropriate. Currently, most Internet standards are published as RFCs, but not all RFCs specify standards. Once a document is assigned a RFC number and published, that RFC is never revised or re-issued with the same number. There is never a question of having the most recent vession of a particular RFC. However, a protocol may be improved and re-documented many times in several different RFCs. The latest "IAB Official Protucol Standards" memo [33] is the reference for determining the correct RFC to refer to for the current specification for each protocol. Every RFC cited has been acquired for the purposes of this thesis.

The IAB has two principal subsidiary task forces: 1) the Internet Engineering Task Force (IETF); and 2) the Internet Research Task Force (IRTF). Each of these task forces led by a chairman and guided by a Steering Committee which reports to the IAB through its chairman.

The IETF is a large open ccmmunity of network designers, operators, vendors and researchers concerned with the Internet and the Internet protocol suite. It is organized 
arrund a set of eight technical areas, each managed by a technical area director. In addition "o the IETF chairman, the area direciors make up the Internet Engineering Steering Committee (IESG) membership. The IAB has delegated to the IESG the general responsibility for making the Internet work and for the resolution of all short- and midrange proticol and architectural issues required to make the Internet function effectively.

To promote research in networking and the development of new technology, the IAB established the Internet Research task Force (IRTF). The IRTF is a community of network researchers, generally with an Internet focus. The work of the IRTF is governed by its Internet Research Steering Group (IRSG).

In the area of network protocols, the distinction between research and engineering is not always clear, so there is sometimes overlap between activities of the IETF and the IRTF. There is considerable overlap in membership between the two groups. This overlup is regarded as vital for cross-fertilization and technology transfer.

\subsection{The Standardization Process}

Within the domain of the Internet and internet technology (DARPA/DoD TCP/IP prot(col suite), a network management framework was defined in RFC1052 (April 88) [1.3] and revised RFC1109 (August89) [14]. RFC1052 resolved the potential competition between three incompatible network management tools that had begun development by the end of 1987. These efforts were the High Level Entity Management System (HEMS) [82], the Simple Gateway Monitoring Protocol (SGMP) [8] and the Common Management 
Information Service/Protorol (CMIS [38] / CMIP [39] ). The latter is an ISO initiative which was adapted to Internet use in a vendor initial-d effort.

The HEMS work was carried out in the context of the Gateway Monituring group of the Internet Engineering Task Force. The SGMP - ffon was carried out largely in the practical context of the NYSERNET and SURAnet regional networks which needed network management facilities to operate satisfactorily [13]. All of the proposals use Abstract Syntax Notation One (ASN.1) [35] for the representation of arbitrary data types.

\subsubsection{RFC1052:[13]}

Two standards for network management were chosen as of RFC1052 [1.3]. The Simple Network Management Protocol (SNMP), RFCl098 [9], an enhanced version of SGMP, was chosen for the short term in order to get the Internet community out of the immediate network management crisis. The other protocol, OSI's CMIP over TCP/IP (CMOT), RFC1095 [99], was designated as the long term solution, contingent on demonstrations that it was suitable for extremely large datagram networks. This implies a (layer 7) application of CMIS/P transferring management information via a TCP/IP lower layer transport service. As well, RFCl052 decided that in order to aid in the eventual transition from SNMP to CMOT, both protocols would be required to use the same management information base (MIB), RFC1066 [7()] and structure of management information (SMI), RFC1065 [71].

\subsubsection{RFC1109:[14]}

RFCl109 was the outcome of the second meeting of the Ad Hoc Network Man- 
agement Review Group which met to consider the state of . . $k$ management for the Internet as of June 1989. Demonstrations of network m...age ..ent tools using SNMP were offered at Interop 88 and the protocol was in use within the Internet as well as private packet networks intemationally.

The common Internet MIB had roughly 100 variables defimtuons (object identifiers), but a typical SNMP monitoring system supported from 100 to 200 additional object identifiers which had been defined in the private or experimental MIB spaces. There was a strong interest in extending the variables accessible via network management tools i.e. adding new devices, new higher level protocols and the ability to manipulate configuration information. CMOT (RFC1095) was based on the ISO Draft International Standard (DIS) of Common Management Information Protocol (CMIP) and no publicly available implementations were known. The University of Wisconsin was deveioping CMOT using ISODE. It was observed that the Internet Structure of Management Information (Internet SMI) was not quite a subset of the ISO CMIS Structure of Management Information (ISO SMI), as the Internet variable naming conventions were a little different. Finally, RFC1 109 recommended to the Internet Activities Board (IAB) that the network management efforts using SNMP and CMOT be allowed independently to explore new variables and potential nonoverlapping Structure of Management Information definitions and that the binding to a common MIB/SMI be relaxed for the next 12 months.

\subsection{Current DARPA Management Standards}

Since that time, the following RFC's have been published. Figure 15 shows the interrelationships of the DARPA standards documents (by title and RFC) that have been 
developed for network management. This diagram was developed by the author after the previous OSI diagrams to show the equivalent roles of each document in its series. This diagram shows that the DARPA standards are not as numerous ur cumplex as the ISO standards documents.

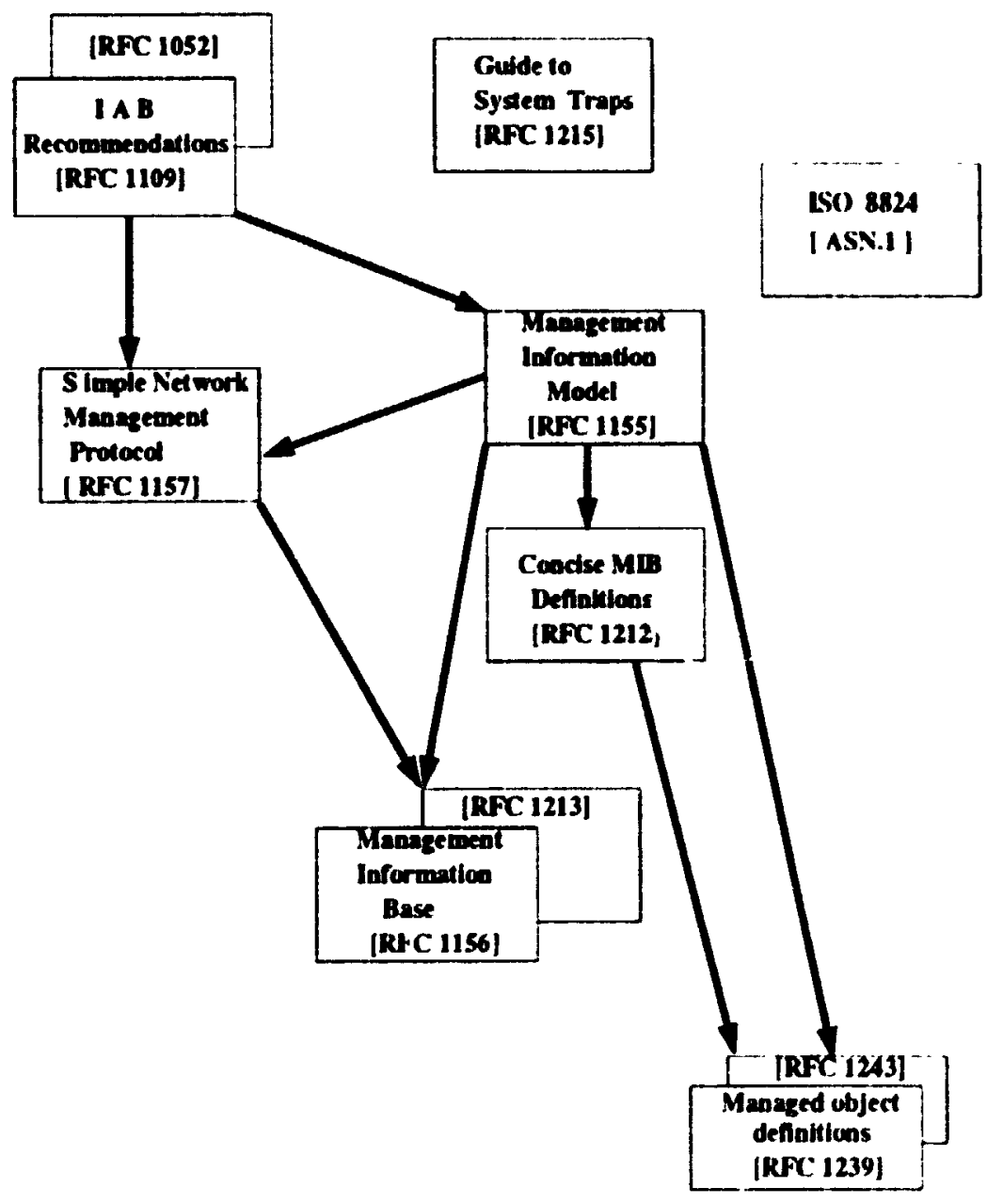

Figure 15 Relationship Between DARPA Standards 


\subsubsection{Services and Protocols}

\subsubsection{RFC1157: SNMP [10]}

RFC1157 [10], the Simple Network Management Protocol defines the legal interactions that can occur between network devices under SNMP. SNMP uses a client-server architecture for monitoring and controlling devices on multivendor TCP/IP networks. It specifies a structure for formatting messages and for transmitting information between reporting devices and data collection programs.

RFCl157 is a re-release of RFCl098 [9], with a changed "Status of this Memo", plus a few minor typographical corrections. The technical content is unchanged from RFC1098. RFC1157 defines a simple protocol by which management information for a network element may be inspected or altered by logically remote users. This memo is consistent with the IAB's diective that the working groups be "extremely sensitive to the need to keep the SNMP simple". The resulting protocol is not backward compatible with its predecessor, the Simple Gateway Monitoring Protocol (SGMP). Although the syntax of the protocol has been altered, the original philosophy, design decisions and architecture remain intact. In order to avoid confusion, new UDP ports have been allocated for use by the protocol described in RFC1157. TCP/IP implementations in the Internet which are network manageable are expected to adopt and implement this specification.

The SNMP Architecture Implicit in the SNMP architecture model is a collection of network management stations and network elements. Network management stations execute management applications which monitor and control network elements. Network 
elements are devices, such as hosts, gateways, terminal servers, and the like, which have management agents responsible for performing the network management funcrions: requested by the network management stations. The SNMP is used to communicate management information between the network management stations and the agents in the network elements.

The first goal of the SNMP is to minimize the number and comple xity of management functions realized by the management agent itself. A second goial of the proficoul is that the functional paradigm for monitoring and control be sufficiently extensihle to accommodate additional, possibly unanticipated aspects of network operation and management. A third goal is that the architecture be, as much as possible, independent of the architecture and mechanisms of particular hosts or particular gateways.

The scope of management information communicated by operation of the SNMP is exactly that represented by instances of all non-aggregate object types either defined in Internet-standard MIB or defined elsewhere according to the conventions set forth in the Internet-standard SMI. Support for aggregate object types in the MIB is neither required for conformance with the SMI nor realized by the SNMP.

Management information communicated by operation of the SNMP is represented according to the subset of the ASN.1 that is specified for the definition of non-aggregate types in the SMI. SNMP utilizes this subset for describing managed objects and for describing the protocol data units used for managing those objects. In addition, the desire to ease eventual transition to OSI-based network management protocols led to the definition in the ASN.I language of the Internet SMI and MIB. The restrictions on the 
use of ASN.I that are part of the SMI contribute to the simplicity espoused and validated y experience with the SGMP. Also for the sake of simplicity, the SNMP uses only a subset of the BER. Namely, all encodings use the definite-length (short or long) form (see previous 2.3.5.1 The Basis Encoding Rules). Further, whenever permissible, primitive ASN.I encodings are used rather than composite encodings (see previous 2.3.5.1 The Basic Encoding Rules). This restriction applies to all aspects of ASN.1 encoding, both for the top-level protocol data units and the data objects they contain.

The SNMP models all management agent functions as alterations or inspections of variables. Thus, a protocol entity on a logically remote host (possibly the network element itself) interacts with the management agent resident on the network element in order to retrieve get) or alter (set) variables. The strategy implicit in the SNMP is that the monitoring of network state at any significant level of detail is accomplished primarily by polling for appropriate information on the part of the monitoring center(s). A limited number of unsolicited messages (traps) guide the tining and focus of the polling. Limiting the number of unsolicited messages is consistent with the goal of simplicity and minimizing the amount of traffic generated by the network management function. Currently, most commands are requests either to set the value of some parameter or to retrieve such a value, and the function of the few imperative commands currently supported is easily accommodated in an asynchronous mode by this manazemers model. In this scheme, an imperative command might be realized as the setting of a parameter value that subsequently triggers the desired action. For example, rather than inıplementing a "reboot command", this action might be invoked by simply setting a 
parameter indicating the number of seconds until system reboot.

The communication of management information among management entities is realized in the SNMP through the exchange of protocol messages. Consistent with the goal of minimizing complexity of the management agent, the exchange of SNMP messages requires only an unreliable datagram service, and every message is entirely and independently represented by a single transport (layer 4 ) datagrarn. While RFCl157 specifies the exchange of messages via the User Datagram Protocul (UDP), the mechanisms of the SNMP are generally suitable for use with a wide variety of transport services.

Because the scope of any SNMP operation is conceptually confined to objects relevant to a single network element, and because all SNMP references to MIB objects are (implicitly or explicitly) by unique variable names, there is no possibility that any SNMP reference to any object type defined in the MIB could resolve to multiple instances of that type. The object instance referred to by any SNMP operation is exactly that specified as part of the operation request or (in the case of a get-next operation) its immediate predecessor in the MIB as a whole.

The names for all object types in the MIB are defined explicitly either in the Internetstandard MIB or in other documents which conform to the naming conventions of the SMI. The SMI requires that conformant management protocols define mechanisms for identifying individual instances of those objects for a particular network element. Each instance of any object type defined in the MIB is identified in SNMP operations by a unique name called its "variable name". In general, the name of a SNMP variable is an OBJECT IDENTIFIER of the form $x . y$, where $x$ is the name of a non-aggregate 


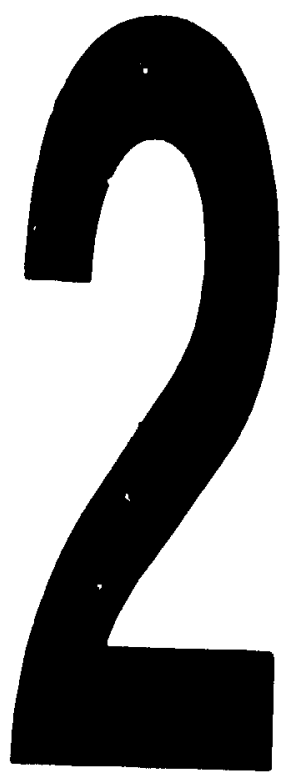

PM-1 3\%"X4" PHOTOGRAPHIC MICAOCOPY TARGET MES 10 Ho AMSI/ISO \&2 EOUIVALENT

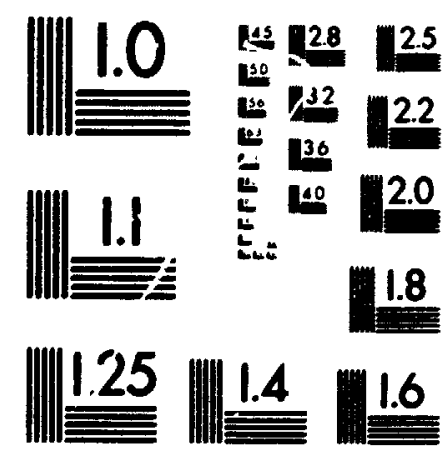

\section{PRECISIONEW RESOLUTION TARGETS}

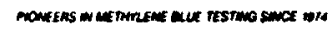

$$
2 \sqrt{25}
$$

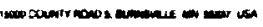

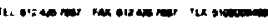


object type defined in the MIB and $y$ is an OBJECT IDENTIFIER fragment that, in a way specific to the named object type, identifies the desired instance. For example, under SNMP in Figure 30, $x$ (for the ieeeBridgeMOC, type $=$ oBridge (1)) has a prefix from the root (see Figure 12) of OBJECT IDENTIFIER = (I.3.6.I.2.I), and a suffix of OBJECT IDENTIFIER $=(o B r i d g e(1))$. The desired instance, $y$ is OBJECT IDENTIFIER $=(\mathrm{aBridgeAddress}(1))$, the value of which makes each oBridge occurrence unique within the value contained in $x$. Therefore the first oBridge would be uniquely identified by $y=a B r i d g e A d d r e s s(1)$ with a value $=1$, the second oBridge would be identified by $y=a$ BridgeAddress $(1)$ with a value $=2$, etc.

Protocol Specification The network management protocol is an application protocol by which the variables of an agent's MIB may be inspected or altered. Communication among protocol entities is accomplished by the exchange of messages, each of which is entirely and independently represented within a single UDP datagram using the BER of ASN.1. A message consists of a version identifier, an SNMP community name and a protocol data unit (PDU). A protocol entity receives messages at UDP port 161 on the host with which it is associated for all messages except fo: those which report traps (i.e. all messages except those which contain the Trap-PDU). Messages which report traps are received on port 162 for further processing. An implementation of this protocol need not accept messages whose length exceeds 484 octets. However, it is recommended that implementations support larger datagrams whenever feasible.

It is mandatory that all implementations of the SNMP support the five PDUs: 
- GetRequest-PDU

- GetNextRequest-PDU

- GetResponse-PDU

- SetRequest-PDU

- Trap-PDU.

Each non-Trap PDU contains a request-id (RequestID), an error status (ErrorStatus), an error index (Errorlndex) and variable bindings (VarBindList). RequestIDs are used .o distinguish among outstanding requests. By use of the Requestld, an SNMP application entity can correlate incoming responses with outstanding requests. In cases where an unreliable datagram service is being used, the RequestID also provides a simple means of identifying messages duplicated by the network. A non-zero instance of ErrorStatus is used to indicate that an exception occured while processing a request. In these cases, Errorlndex may provide additional information by indicating which variable in a list caused the exception.

The term variable refers to an instance of a managed object. A variable binding, or VarBind, refers to the pairing of the name of a variable to the variable's value. A VarBindList is a simple list of variable names and corresponding values. Some PDUs are concerned only with the name of a variable and not its value (e.g. the GetRequest-PDU). In this case, the value portion of the binding is ignored by the protocol entity. However, the value portion must still have valid ASN.1 syntax and encoding. It is recommended that the ASN.1 value NULL be used for the value portion of such bindings. 


\subsubsection{DARPA Structure of Management Information (SMI)}

\subsubsection{Abstract Syntax Notation One (ASN.1): ISO 8824 [35]}

The desire to ease eventual transition to OSI-based network management protocols led to the definition of the Internet SMI and MIB in the ASN.1 language. Management information communicated by operation of the SNMP is represented according to the subset of the ASN.1 [35] that is specified for the definition of non-aggregate types in the SMI. Also for the sake of simplicity, the SNMP uses only a subset of the BER [36].

\subsubsection{RFC1155:Structure of Management Information [73]}

$\mathrm{RFC1155}$ [73] is a re-release of RFC1065, with the changed "Status of this Memo", plus a few minor typographical corrections. RFC1155 provides the common definitions for the structure and identification of management information for TCP/IP-based internets. The status of this memo is "Recommended" and TCP//P implementations are expected to adopt and implement this specification. Included are descriptions of an object information model for network management along with a set of generic types used to describe management information. Managed objects are accessed via a virtual information store, termed the Management Information Base (MIB). Objects in the MIB are defined using ASN.1. Each type of object has a name, a syntax and an encoding.

Names Hierarchical names are used to identify managed objects. The ASN.1 OBJECT IDENTIFIER concept is used to model this notion. OBJECT IDENTIFIERs are a means for identifying some object, regardless of the semantics associated with the object. An OBJECT IDENTIFIER is a sequence of non-negative integers which traverse a global 
tree. This is the same global tree as discussed previously in the OSI context. In Figure 12, under the iso(l) node, the ISO has designated one subtree for use by other (inter)national organizations, identified-organization(3). Of the children nodes present, two have been assigned to the U.S National Institutes of Standards and Technology. One of these subtree has been transferred by the U.S. NIST to the U.S. Department of Defense, dod(6). RFC1155 assumes that DoD will allocate a node to the Internet community, internet(I), to be administered by the IAB. Therefore, the Internet subtree of OBJECT IDENTIFIERS starts with the prefix: 1.3.6.l). The Internet subtree of RFC1156-mib Managed OBJECT IDENTIFIERS starts with the prefix: 1.3.6.1.2.1 (see Figure 12). In the SNMP side of Figure 30 is one of the leaf ends of the Internet subtree. This shows that the IEEE 802.1 LAN Bridge Managed Object Class, ieeeBridgeMOC, is defined by the node (I). This diagram also shows that a IEEE LAN bridge port table object is defined as oBridge(32) (see oBridgePortTable in Figure 30), and the IEEE LAN bridge port number, aPortNumber, OBJECT IDENTIFIER is defined by oBridge(32) oBridgePortTable (0) oPortEntry (1) (see oPortNumber in Figure 30).

Syntax Syntax is used to define the structure corresponding to object types. ASN.1 constructs are used to define this structure, although the full generality of ASN.1 is not permitted. The ASN.1 type ObjectSyntax defines their different syntaxes which may be used in defining an object type.

Only the ASN.1 primitive types INTEGER, OCTET STRING, OBJECT IDENTIFIER and NULL are permitted. The ASN.1 composite type SEQUENCE is permitted, providing that it is used to generate either lists or tables. For lists, the syntax takes the 
form: SEQUENCE (<type l>, ..., <typeN> | where each type> resolves to one of the ASN.1 primitive types listed above. For tables, the syntax takes the fomi: SEQUENCE OF entry> where <entry> resolves to a list composite.

Encodings Once an instance of an object has been identified, its value may be transmitted by applying the BER of ASN.1 to the syntax for the object type (see 2.3.5.1 The Basic Encoding Rules).

Managed Objects Although it is not the purpose of RFCl155 to define objects in the MIB, it does specify a format to be used by other RFCs which define these objects. This is similar to the role of the GDMO in the OSI context. Figures 25 and 26 are tables of the IEEE 802.1 LAN Bridge Managed Objects. An RFC1155 object definition consists of five fields:

- Object: a textual name, termed the OBJECT DESCRIPTOR, for the object type, along with its corresponding OBJECT IDENTIFIER.

- Syntax: the abstract syntax for the object type. This must resolve to an instance of the ASN.1 type ObjectSyntax.

- Definition: a textual description of the semantics of the object type. Implementations should ensure that their instance of the object fulfills this definition since the MIB is intended for use in multi-vendor environments. As such it is vital that all objects have consistent meaning across all systems.

- Access: one of read-only, read-write, write-only or not- accessible.

- Status: one of mandatory, optional or obsolete. 
Guidelines for Object Names Each OBJECT DESCRIPTOR corresponding to an object type in the Intemet-standard MIB shall be an unique, but mnemonic, printable string. This promotes a common language for humans to use when discussing the MIB and also facilitates simple table mappings for user interfaces.

Object Types and Instances An object type is a definition of a kind of managed object; it is declarative in nature. In contrast, an object instance is an instantiation of an object type which has been bound to a value. For example, the notion of an entry in a routing table might be defined in the MIB. Such a notion corresponds to an object type; individual entries in a particular table which exist at some time are object instances of that object type.

A collection of object types is defined in the MIB. Each such subject type is uniquely named by its OBJECT IDENTIFIER and also has a textual name, which is its OBJECT DESCRIPTOR. The means whereby object instances are referenced is not defined in the MIB. Reference to object instances is achieved by a protocol- specific mechanism; it is the responsibility of each management protocol adhering to the SMI to define this mechanism.

An object type may be defined in the MIB such that an instance of that object type represents an aggregation of information also represented by instances of some number of subordinate object types. An object type defined in this way is called a list. Similarly, tables can be formed by aggregations of a list type.

Each management protocol must provide a mechanism for accessing simple (non- 
aggregate) object types. Each management protocol specifies whether or not it supports access to aggregate object types. Further, the protocol must specify which instances are "retumed" when an object type/instance pairing refers to more than one instance of a type. To afford support for a variety of management protocols, all information by which instances of a given object type may be usefully distinguished, one from another, is represented by instances of object types defined in the MIB.

3.3.2.3 RFC1156: MB-I [72] RFC1156 [72] is a re-release of RFC1066, with a changed "Status of this Memo", "IAB Policy Statement", and "Introduction" sections plus a few minor typographical corrections. The technical content is unchanged from RFC1066. RFC1156 provides the initial version of the Management Information Base (MIB) for use with network management protocols in TCP/IP-based internets in the short-term. The MIB defines the actual management objects which are operated upon by the management protocol. It also defines what protocol operations may be applied to each object. For example, it defines an object that represents TCP Connection Blocks (TCBs) and decrees that remote applications can read but not alter this object. TCP/IP implementations in the Internet which are network manageable are expected to adopt and implement this specification. The initial draft of RFC1156 was heavily influenced by the HEMS [82] and SNMP [9] MIBs.

The IAB urged the working groups to be "extremely sensitive to the need to keep SNMP simple". Thus the list of managed objects defined in RFC1156 was derived by taking only those elements which were considered essential (i.e. only 126 management objects are defined). Since such elements are essential, there was no 
need to allow the implementation of individual objects to be optional. Rather, all compliant implementations will contain all applicable objects defined in RFC1156. All implementations must provide certain types of functionality such as routing tables and protocol control blocks.

Objects Groups Since there is no need to allow individual objects to be optional, the managed objects are arranged in the following eight groups: System, Interfaces, Address Translation, IP, ICMP, TCP, UDP and EGP. There are two reasons for defining these groups: 1) to provide a means of assigning object identifiers; All object identifiers contain a group specific prefix (e.g. "if" for interfaces group). 2) to provide a method for implementations of managed objects to know which objects they must implement. This method is is follows: if the semantics of a group is applicable to an implementation, then it must implement all objects in that group. For example, an implementation must implement the TCP group if and only if it implements the TCP protocol. RFCl156 does not include management information for applications.

Systems Croup (prefix "sys") The System group is used to represent information about the system on which the protocol suite is running, in particular, information about the system's manufacturer and software revision, as well as how long the system has been up. Implementation of the Systems group is mandatory for all systems.

Interfaces Group (prefix “ir') The Interfaces group presents generic information about each network interface (layer 2) on the system. A large amount of information is stored about each interface, including the number of inbound and outbound data errors found, 
the number of broadcast, multicast and unicast packets sent. the number of packets received and sent, and the maximum transmission unit of the device. In practice this definition has proved inadequate for device specific information such as Ethernet frame errors. Implementation of the Interfaces group is mandatory for all systems.

Address Translation Group (prefix "at") The Address Translation group contains the mappings between IP addresses and subnetwork-specific addresses that all IP systems must support. Implementation of the Address Translation group is mandatory for all systems.

Internet Protocol Group (prefix "ip") The IP group stores information about the Internet Protocol (IP) layer (layer 3). This information is generally the same as the information kept for interfaces (e.g. datagrams received and sent, counts of datagrams with errors, etc.). In addition, the layer contains addressing information as well as control variables that permit remote applications to adjust the default IP Time-to-Live and manipulate the IP routing tables. Implementation of the IP group is mandatory for all systems.

\section{Internet Control Message Protocol Group (prefix "icmp")}

The ICMP group stores information about the (ICMP) layer (layer 3). This information includes aggregate counts of datagrams received and sent, counts of particular types of messages received. Individual counters for ICMP message (sub-kcodes have been omitted from this version of the MIB for simplicity. Implementation of the ICMP group is mandatory for all systems. 


\section{Transport Control Protucot Group (prefix "icp")}

The TCP group stores information about the (TCP) layer (layer 4). This information includes aggregate counts of datagrams received and sent, counts of particular types of messages received and protocol control blocks. The instances of object types that represent information about a particular TCP connection are transient; they persist only as long as the connection in question. This is problematic because if a user complains to a network manager that a connection has failed, the information that the manager wants most is the old control block for the broken connection because it contains all the state information for the connection at the time that it failed. Implementation of the TCP group is mandatory for all systems that implement the TCP protocol.

\section{User Datagram Protocol Group (prefix "udp")}

The UDP group stores information about the UDP layer (layer 4). This information includes aggregate counts of datagrams received and sent, counts of particular types of messages received. Implementation of the UDP group is mandatory for all systems which implement the UDP protocol.

\section{Exterior Gateways Protocol Group (prefix "egp")}

The EGP group stores information about the Exterior Gateways Protocol (layer 4), the routing protocol used to link autonomous systems. Implementation of the EGP group is mandatory for all systems which implement the EGP protocol.

In addition, vendors can define extensions to the MIB, which allows the vendor to support features particular to their devices yet remain compatible with other vendors' 
SNMP devices. As we l, research organizations can define extensions in the "experimental" tree of the :MIB.

3.3.2.4 RFC1213: (MIB-II) [74] RFC1213: (MIB II) [74] defines the second and most current version of the Management Information Base (MIB-II) for use with network management protocols in TCP/IP-based internets. In particular, together with its companion memos which describe the structure of management information (RFC 1155) along with the network management protocol (RFC 1157) for TCP/IP-based internets, these documents provide a simple, workable architecture and system for managing TCP/IP-based internets and in particular the Internet community.

Management Information Base defines a minimum set of 100 definitions of variables, test points and controls that must be supported.

Changes from RFC1156:[72] Features of this MIB include:

- incremental additions to refiect new operational requirements;

- upwards compatibility with the SMIMIB and the SNMP;

- improved support for multi-protocol entities; and,

- textual clean-up of the MIB to improve clarity and readability.

Systems Group (prefix "sys") Four new objects are added to this group:

- sysContact

- sysName

- sysLocation 
- sysServices

These provide contact, administrative, location, and service information regarding the managed node.

Interfaces Group (prefix "if") The ifTable object was mistakenly

marked as read-write, it has been (correctly) re-designated as not-accessible. In addition, several new values have been added to the ifType column in the ifTable object:

- $\quad \operatorname{ppp}(23)$

- softwareLoopback(24)

- $\operatorname{con}(25)$

- ethernet-3Mbit(26)

- $n s i p(27)$

- $\operatorname{slip}(28)$

- ultra(29)

- $d s 3(30)$

- $\operatorname{sip}(31)$

- frame-relay(32)

Finally, a new column has been added to the ifTable object:

ifSpecific

which provides information about information specific to the media being used to realize the interface. 
Address Translation Group (prefix "at") In MIB-I this group contained a table which permitred mappings from network addresses (e.g., IP addresses) to physical addresses (e.g., MAC addresses). Experience has shown that efficient implementations of this table make two assumptions: a single network protocol environment, and mappings nccur only from network address to physical address.

In order to meet both the multi-protocol and inverse mapping requirements, MIB-II and its successors will allocate up to two address translation tables inside each network protocol group. That is, the IP group will contain one address translation table, for going from IP addresses to physical addre:ses.

Internet Protocol Group (prefix "ip") The access attribute of the variable ipForwarding has been changed from read-only to read-write. In addition, there is a new column to the ipAddrTable object.

\section{ipAdEntReasmMaxSize}

which keeps track of the largest IP datagram that can be re-assembled on a particular interface.

The descriptor of the :pRoutingTable object has been changed to ipRoureTable for consistency with the other IP routing objects. There are also three new columns in the ipRouteTable object,

- ipRouteMask

- ipRouteMetric5

- ipRouteInfo 
the first is used for IP routing subsystems that support arbitrary subnet masks, and the latter two are IP routing protocol-specific.

Two new objects are added to the IP group:

- ipNetToMediaTable

- ipRoutingDiscards

the first is the address translation table for the IP group (providing identical functionality to the now deprecated atTable in the address translation group), and the latter provides information when routes are lost due to a lack of buffer space.

Internet Control Message Protocol Group (prefix “icmp")

There are no changes to this group.

Transport Control Protocol Group (prefix "tcp") Two new variables are added: tcpInErrs tcpOutRsts

which keep track of the number of incoming TCP segments in error and the number of resets generated by a TCP.

User Datagram Protocol Group (prefix “udp") A new table: udpTable is added

\section{Exterior Gateways Protocol Group (prefix “egn")}

Experience has indicated a need for additional objects that are useful in EGP monitoring. In addition to making several additions to the egpNeighborTable object, i.e., - egpNeighAs 
- egpNeighInMsgs

- egpNeighInErrs

- egpNeighOutMsgs

- egpNeighOutErrs

- egpNeighInErrMsgs

- egpNeighOutErrMsgs

- egpNeighStateUps

- egpNeighStateDowns

- egpNeighIntervalHello

- egpNeighIntervalPoll

- :gpNeighMode

- egpNeighEventTrigger

A new variable is added:

egpAs

which gives the autonomous system associated with this EGP entity.

SNMP Group (prefix "snmp")

The application-oriented working groups of the IETF have been tasked to be receptive towards defining MIB variables specific to their respective applications.

For the SNMP, it is useful to have statistical information. A new group, the SNMP group, is allocated for this purpose:

snmp OBJECT IDENTIFIER ::=\{ mib-2 11$\}$ 
3.3.2.5 RFC1214: OIM-MIB II [67] At the IETF OSI Internet Management (OIM) Working Group meeting of $90-12-4$, no objections or major corrections were offered. The Internet SMI was based on a very early draft of the ISO SMI. The ISO SMI continued to evolve as early problems were resolved. The Internet SMI did not keep pace. The ISO SMI is now stable and required by most OSI-based management systems. Unfortunately most of the MIBs being defined within the IETF are only satisfying the requirements of the IETF SMI, not taking into account the minor additional requirements for OSI management. This requires additional work to map these IETF SMI-based MIBs into ISO SMI. This is what the OMM-MIB-II document [67] does for MIB-II (RFC1213).

\subsubsection{RFC1243: Appletalk MIB [97] RFC1243 [97] defines a portion of the Man-} agement Information Base (MIB) for use with network management protocols in TCP/IPbased internets. In particular, it defines objects for managing AppleTalk networks. This memo is a product of the AppleTalk-IP Working Group of the Internet Engineering Task Force (IETF).

Overview Apple Talk is a protocol suite which features an open peer-to-peer architecture that runs over a variety of transmission media. AppleTalk is defined in [90]. This protocol suite interoperates with the IP protocol suite through various encapsulation methods. As large AppleTalk networks are built that coexist with large IP networks, a method to manage the AppleTalk networks with SNMP becomes necessary. This MIB defines managed objects to be used for managing AppleTalk networks. 
This AppleTalk MIB defines about 80 network management variables. Some 10 vendors are already using them to build agent software that can be communicated with management systems based on SNMP. With this agent software, network managers will be able to obtain management information for port configuration on routers and host computers, network numbers and node numbers, type of AppleTalk network and the performance of protocols such as Apple Datagram Delivery Protocol (DDP). The MIB would also provide information on the performance and status of printing protocols or other upper layer protocols.

Structure of MIB The objects are arranged into the following groups:

- LLAP - LocalTalk Link Access Protocol

- AARP - AppleTalk Address Resolution Protocol

- ATPort -AppleTalk Port

- DDP - Datagram Delivery Protocol

- RTMP - Routing Table Maintenance Protocol

- KIP - Kinetics Internet Protocol

- ZIP - Zone Information Protocol

- NBP - Name Binding Protocol

- ATEcho - AppleTalk Echo Protocol

These groups are the basic unit of conformance. If the semantics of a group is applicable to an implementation, then it must implement all objects in that group. For example, a managed agent must implement the KIP group if and only if it implements the 
KIP protocol. These groups are defined to provide a means of assigning object identifiers, and to provide a method for managed agents to know which objects they must implement.

LocalTalk Link Access Protocol Group (prefix "llap") The LocalTalk Link Access Protocol (LLAP) is a medium-speed data-link protocol designed for low cost and plugand-play operation. The LLAP group is designed to manage all interfaces on a managed device that use this protocol.

AppleTalk Address Resolution Protocol Group (prefix "aarp") The AppleTalk Address Resolution Protocol (AARP) is used to map between AppleTalk node addresses, used by the Datagram Delivery Protocol, and the addresses of the underlying Data Link layer. The AARP table allows for management of the Address Mapping Table on the managed device.

\section{AppleTalk Port Group (prefix "atport")}

An AppleTalk Port is a logical connection to a network over which Apple Talk packets can be transmitted. This group allows the management of the configuration of these Apple Talk ports.

Datagram Delivery Protocol Group (prefix “ddp") The Datagram Delivery Protocol (DDP) is the network-layer protocol that is responsible for the socket-to-socket delivery of datagrams over the AppleTalk Internet. This group manages the DDP layer on the managed device. 
Routing Table Maintenance Protocol Group (prefix "rtmp") The Routing Table Maintenance Protocol (RTMP) is used by AppleTalk routers to create and maintain the routing tables that dictate the process of forwarding datagrams on the AppleTalk internet. The RTMP group manages the RTMP protocol as well as the routing tables generated by this protocol.

Kinetics Internet Protocol Group (prefix “kip") The Kinetics Internet Protocol (KIP) is a protocol for encapsulating and routing AppleTalk datagrams over an IP internet. This name is historical. The KIP group manages the KIP routing protocol as well as the routing tables generated by this protocol.

Zone Information Protocol Group (prefix "zip") The Zone Information Protocol (ZIP) is used to maintain a mapping between networks and zone names to facilitate the name lookup process performed by the Name Binding Protocol. The ZIP group manages this protocol and the mapping it produces.

Name Binding Protocol Group (prefix "nbp") The Name Binding Protocol (NBP) is a transport-level protocol that is used to convert human readable service names into the numeric AppleTalk network addresses needed for communicating across the Apple Talk network. The NBP group manages this protocol and the NBP services that exist on the managed device.

AppleTalk Echo Protocol Group (prefix "atecho") The AppleTalk Echo Protocol is a transport-level protocol used to test and verify the status of the AppleTalk internet. The AtEcho group manages this protocol. 


\section{CHAPTER 4 Comparing OSI and DARPA}

\subsection{Comparing OSI and DARPA Protocol Stacks}

Before comparing the OSI and DARPA network management standards, Figure 16 compares the two protocol stacks against a generic 7-layer OSI Basic Reference Model.

USER
\begin{tabular}{|l|}
\hline Application \\
\hline Presentation \\
\hline Session \\
\hline Transport \\
\hline Network \\
\hline Data Link \\
\hline Physical \\
\hline
\end{tabular}

O S I

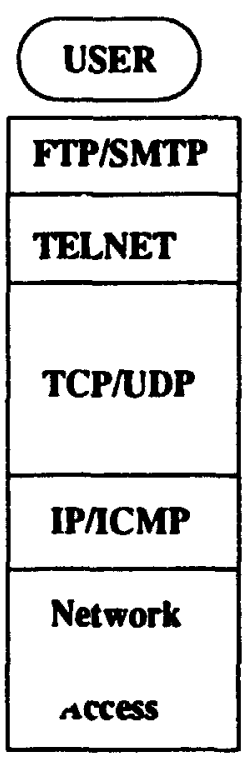

D A R P A

Figure 16 Comparing OSI and DARPA Protocol Stacks

Layers 1 and 2 allow interconnection between nodes and compares OSI Physical and Data Link protocols with DARPA Network Access protocols. Layer 3 allows 
internetworking between nodes and compares OSI Network protocols such as X.25 with DARPA Internet Protocol (IP). Layer 4 allows connection and connectionless end-to-end data transfer and compares OSI Transport protocols such as TP4 and TP0 with DARPA Transport Control Protocol (TCP) and User Datagram Protocol (UDP). Layer 7 allows interoperability between peer applications such as Email and compares OSI Application protocols such as FTAM, X.400 and CMIP against DARPA FTP, SMTP and SNMP.

\subsection{Comparing CMIS/P and SNMP}

Until fairly recently, there have been debates comparing the two sets of protocols, de jure standards-based CMIS/P and de facto standards-based SNMP, for dominance of the heterogeneous network management marketplace. OSI proponents claim that CMIP

offers more functionality and would eventually be a true standard. But SNMP proponents have pointed out that, unlike CMIP, SNMP products are already defined and available. SNMP is distinguished from CMIS/P in two important ways. One, SNMP operates over UDP/IP, so it requires only a four-layer protocol stack to support network management. Two, SNMP is connectionless, or datagram-based, which means that a session need not be established before network management information can be sent. "SNMP offers the additional advantage of flexibility; whereas CMIS/P can only manage OSI objects, SNMP can be used to manage virtually any object, even OSI objects," [20]. SNMP is defined for use only on TCP/IP networks over Ethemet; and not currently for token-ring media, or for use under other protocols. The reality is that networks today are mixtures of multiple protocols and topologies. 
The essence of the comparison is based on arguing the cost/benefit trade-offs of having simpler or more complex underlying primitives. This difference can be more concisely stated as the "complete aspects" of CMIP versus the"core aspects" of SNMP. From the network operator's perspective, the protocols are equivalent, as the same ne'work management application can be built upon either one. In fact, equivalent functionality can be provided using both protocols if a mixed set of management agents is empioyed

Data access philosophy CMIP is oriented toward retrieving aggregate information; SNMP is oriented toward retrieving individual items of information. For simple queries between the manager and the agent the two protocols provide roughly the same functionality. It has been argued that the extra load on the agent required by CMIP is unwarranted. However, the use of the unique scoping and filtering capabilities within CMIP allows more complicated queries. The same functionality can be achieved by SNMP but requires more queries and more information load on the network.

For dealing with individual information objects, SNMP is more efficient. The CMIP approach is to retrieve the whole database and discard everything that is not required. That is not very efficient for dealing with individual information objects. However, the future of global internetworking will present many orders of magnitude more information objects than today. Isolating individual objects by iterative filtering will be a necessary capability that only CMIP promises to have.

Polling versus reporting From a network bandwidth perspective, CMIP tends to cause less background load on the network during normal operations because the agents 
typically monitor themselves and send event reports in case of trouble. SNMP is less efficient in terms of bandwidth consumption as a manager needs to query the agents for their status more often. This should come as no surprise as SiNMP was developed expecting to handle dozens to perhaps hundreds of devices scattered across a global network, where the added traffic was not significant. On most LANs this would not be a problem. But as the network increases from hundreds to thousands and tens of thousands of devices, the bandwidth needed for SNMP activity can degrade WAN service to user traffic. The scalability of SNMP is in question, whereas the initial cost in overhead and the current unavailability of implementations put CMIP in question.

However, the bandwidth load from SNMP is more predictable and more controllable. This is an additional factor in DARPA network design for which network designers can compensate. Another design decision required with SNMP is to determine how often to poll for network status information. As well, the time-out value for each device is variable because the time needed to obtain a response depends on the distance (i.e. in intemet hops) from the manager or proxy.

Functionality CMIP is generally thought of as having more built-in specific features and capabilities than SNMP. Mechanisms such as scoping, filtering and synchronization allow the retrieval of all attributes in a sub-tree in one CMIP request. However, it can be argued that these require more expensive development and operational resources, while the required funciionality can be accomplished by using SNMP itself. For alarm reporting, both and SHMP have open-ended syntax, which allows vendors to support their own events. CMIP proxies can support an event sieve and event log file. CMIP 
generic notifications include a lot of useful information such as severity, trend indicator, problem type, etc.

Size and performance Some concem has been expressed about the resources needed to implement CMIP. Implementation of a full OSI stack in addition to management instrumentation is problematic on smaller network nodes. Users want network management capabilities, but not if it substantively increases price or decreases performance. This a the main argument of the developers of SNMP. SNMP's simplicity gives better cost/performance.

Transport layers For end-to-end transport, SNMP utilizes a connectionless service such as UDP (User Datagram Protocol). This means that there is no guarantee of delivery. If the receiving device does not acknowledge receipt, the sending device simply retransmits the message. The drawback of UDP is that there is no end-to-end error correction. If an SNMP trap is lost, then the manger loses its synchronization with the device.

In comparison, CMIP requires connection-oriented transport such as TCP/IP. The drawback is the overhead that this type of transmission mechanism can produce, both in the network and in the application processes. While the reliable transport makes CMIP better at retrieving large amounts of data, it may also make the network harder to manage when trouble occurs. If the network is in a fault state, use of unreliable transport allows the network management station to retry until transmission is successful. A connection- 
oriented network may not be able to deal with that and it may not be able to get a connection in the first place.

Structure of Management Information (SMI)

Both CMIP and SNMP use ASN.1 and the BER to represent management information. Only a subset of the basic types are permitted in the SNMP SMI, which does not allow the definition of complex attributes. In particular, a scalar object ultimately resolves to either an INTEGER, OCTET STRING, or OBJECT IDENTIFIER. By omitting support for arbitrarily complex data types, substantive savings can be achieved in terms of program development, code size and execution time. On the other hand, CMIP supports the full richness of the ASN.1 definitions. The major drawback is that the complexity is not hidden in lower protocol layers. Therefore, the massive use of the type "ANY DEFINED BY" has the consequence that the upper application layer must implement ASN.I parsers and formatters, which must be written for each type.

Modeling It is worth noting that CMIP allows greater structure to be placed on the objects contained in the MIB. The issue is the relative merits of defining MIB variables in ISO GDMO format versus standard Internet format. That is, the formats being compared are ISO GDMO "templates" [ISO 10165-4] and OBJECT-TYPE macrus [RFC 1155]. There are many technical considerations to be taken into account when mapping the OSI SMI and the Internet SMI. The OBJECT-TYPE approach is essentially a tiny subset of the GDMO approach. The GDMO language is vastly richer - indeed some might argue, too rich. Perhaps the correct approach is to define new MIB objects with GDMO and let 
the Internet versions (subsets) be derived from them. Both models agree that the MIB is a set of values (e.g. variables in the OBJECT-TYPE model and attributes in the GDMO model). Each value has a type (e.g. object type, attribute type) which associates it with an object identifier, a syntax (specified in ASN.1) and a set of allowed operations (e.g. read, write, add elements, etc.). Both protocols allow for vendor-specified extensions to the MIB.

The differences concern the mechanism for naming specific value instances and groups of instances. The OBJECT-TYPE model (SNMP) assumes that individual variables also have object identifiers, which are formed by adding a suffix to the Object Identifier (OD) of the object type. It further assumes that "closely related" attributes have OID's that are "close" in the sense that they differ only in the last few components. This is illustrated in the left-hand side of Figure 30. By contrast, the GDMO model explicitly groups related attributes into "objects" and names instances with a different technique based on database-like associative queries. The result is that for GDMO, object identifiers are totally uninterpreted "atomic" values. This is illustrated in the righthand side of Figure 30. If one is designing a GDMO definition that is also to be useful in the OBJECT-TYPE world, one constraint concerns selection of object identifiers. Attributes of a class should all contain the id of the class as a prefix (with the exception of the NAMING attribute, if added). On the other hand, the naming mechanism may require new "key" attributes to be defined (i.e. NAMED BY).

The rest of GDMO fall into two general categories: support for functionality not provided by SNMP and convenience features for abbreviating definitions. ACTIONS 
and EVENTS can be placed in the first category. The OBJECT-TYPE version may have to add "phoney" attributes to simulate ACTIONS (e.g. a variable that can be set to cause a device to reset itself as a side effect). Inheritance, group attributes and packages can be placed in the second. GDMO provides the capability of adding "conditional" attributes, notifications, actions and behaviour to OSI objects in the form of CONDITIONAL PACKAGES. Which conditional packages are actually present in an object instance is contained in the "packages" attribute inherited from the TOP object class. No special relationship attribute is required to relate the conditional package to the object. This is contrary to the Internet practice of defining multiple optional groups, including tables, where relationships are explicitly expressed using "indexes".

For example, the IETF LAN Bridge proposal uses multiple tables, with the relationship to individual ports captured by a port number for each entry (see Figure 30). Some of the tables are optional, depending on whether the spanning tree or source routing is implemented. The GDMO version consolidates these multiple tables into one table containing entries on a per port basis (see Figures 28 and 30 ).

Another issue is that all objects defined in GDMO inherit five mandatory attributes from TOP. Three of these attributes are SET-valued, which cannot be manipulated by SNMP. In GDMO, notifications (traps) are defined as part of an object class. It might be desirable to assure that a mapping of such notifications into SNMP traps is possible. It is not clear to which category allomorphism belongs. 


\section{Layer Managed Objects}

In order for implementors to develop interoperable network management products, profile agreements for layer managed objects are required. The OSI process of developing layer agreements is likely to take several years. The Transport and Network Layer management standards under development by ISO/IEC/JTC1/SC6 have recently reached DIS. The Lower Layer Special Interest Groups (LLSIG/EG-LL) of the regional workshops have begun development of implementation agreements for Transport and Network.

By comparison, this was accomplished in DARPA by RFC1156 [72] in 1990. The shorter DARPA timeframe reflects both the difference in orders of magnitude of scope between the DARPA and OSI frameworks; and the difference in philosophy between DARPA's narrow delegation (de facto) and OSI's international consensus (de jure).

Security As the interconnection of networks from different domains increases, security of the information being transferred, and of the networks themselves, will become important issues. Only recently is security becoming a requirement of SNMP implementations. SNMP is considered a vulnerability because the agent responses to manager polls contain network elements' full network address. An IETF Task Force has begun to address security for SNMP. On the other hand, security has always been recognized as a requirement for OSI network management [49] [59].

Coexistence It would have been better to have only one standard protocol, but the reality is that both will be used for network management for the foreseeable future. Thus, in the not too distant future, we will see SNMF and CMIP manageable devices 
coexist in the same network and the demand for true integrated network management systems will increase.

However, it is still not clear how consistent end-to-end management is applied on a mixed SNMP and CMIP network. To be more specific, as discussed above, the structure of the MIB defined by SNMP community is very different from that defined by CMIP community. For example, if a network manager wants to restart a device in a network. a "restart" operation will be invoked on the CMIP-based device, but a variable will be set to TRUE or "l" on the SNMP-based device.

There are two general approaches to coexistence:

- Managers with dual stacks

- Manager of Managers (MOM), where an SNMP Manager is MANAGED by a CMIP agent.

Dual Stack Some vendors have started to address the issue by providing a dual stack on their network management systems (i.e., the manager). In the dual stack approach, most vendors have defined a common API (Âppilication Programming Interface) on top of the SNMP and CMIP protocol stacks, so that the differences between the service primitives (using the OSI terminology) can be hidden from the management applications.

However, allowing the manager to communicate simultaneously with SNMP and CMIP devices doesn't really solve the integration problem. Because of the differences in MIB structures, the applications still have to deal with two types (semantics) of managed objects. 


\section{Manager of Managers (MOM)}

In the long term, it is visualized that within some networks, levels of network management may exist. In these cases, the network manager directly linked to the managed object mav only perform part of the network management function, with the remainder located at another location performed by a different manager. What is starting to emerge is a hierarchy (MOM). The vision is a two-tiered hierarchy, with OSI occupying the top tier (e.g. global or corporate layer) and SNMP or OSI providing network management solutions on a national, regional or local level fitting into the umbrella system (see Figure 24).

In the "MOM" scenario, the approach is similar to the dual stack approach, except that the dual stack is now implemented in the SNMP manager. The SNMP manager acts as an element manager for the CMIP-based network manager. The SNMP manager exchanges SNMP protocol with the devices on the network and exchanges CMIP (through the CMIP agent) to the "enterprise" manager. The management applications in the enterprise manager only need to understand CMIP objects. However, the SNMP manager is now responsible for translating the SNMP object into the CMIP object.

Transition The MIB and the SMI, which SNMP uses, were designed to be compatible with the OSI direction (via ASN.1) and to work jointly with a future stable OSI network management solution. The introduction of ASN.1 to the Internet is a fundamental provision, and together they should allow future applications to be portable between environments. 
DARPA has made a commitment to transition from TCP/IP architecture and protocol standards to the OSI architecture and OSI-related international standards [93]. Although there has been no official schedule published, the general strategy for this transition has emerged. The plan is phased. First, OSI is introduced "stand alone" to ensure that functionality is comparable to TCP's. Next, OSI and TCP systems interwork using the layer 7 getaways. Dual stacks are used throughout the transition where traffic volumes dictate. The last phase is to move the network subscribers to OSI as they buy new systems or install major upgrades. Because of the eventual migration from TCP/IP to OSI, CMIP is clearly the network management solution for the long term. Therefore the scope of this transition strategy must be increased to include network management and allow for the protection of current investments in SNMP and migration to CMIP smoothly in the future.

Conclusion Because of the availability of SNMP products, SNMP is the appropriate solution for networks with an immediate need for network management. In its "1990 SNMP Product Guide", Datapro Research says that $71 \%$ of such users believe SNMP will be the dominant intemetwork network management protocol for at least three to five years, and $23 \%$ believe it will be around for six to ten years. SNMP is proving so popular, in fact, that even network vendors whose products are not intended for TCP/IP user traffic are implementing SNMP with the expectation that users will add TCP/IP to their network for management protocol traffic. SNMP provides use:s and vendors with a good first cut at open, multi-vendor network management, which can be brought up without major changes to networking applications. SNMP provides a working environment to 
help explore the behaviour and management needs of big, distributed, business-critical networks, comprising hundreds and thousands of devices. Users can learn about the impact of management on networks, and what the network manager really needs.

The reality is that both protocols will occipy certain segments of the market, and that a solution to accommodate both is needed to achieve true integrated end-to-end network management. The important requirement from the interoperability point of view is that the instrumentation in each agent have the same semantics, irrespective of which network management framework is used to access it.

Both CMIP and SNMP have the same goal of moving information from one place to another so that a network manager can find out what is broken. It does not really make much difference to a user what the underlying protocol is, as long as it does the job. OSI network management will have the same obstacles to overcome, vis a vis SNMP, that the general OSI protocols have in supplanting TCP/IP. CMIP will have to make economic sense, it must be fully interoperable and it must exceed SNMP functionality. SNMP will be replaced when these requirements are met. The important thing from the network manager point of view is that standards, per se, are of no interest to users without robust products which implement these standards.

En route to the deployment of the OSI MIB and consolidation of network management systems around the OSI standards, SNMP is a perfect facilitator and a catalyst. In other words, for open multivendor management, SNMP is the simple answer for today's networks. Figure 17 illustrates the comparison between OSI and SNMP. The conclusion of the comparison can be inferred in the last two rows which depict OSI as comprehen- 
sive- but developing, and SNMP as simple-but developed. These represent the bottom lines in the current debate.

OSI

ISO 10165 • 4 [ GDMO ]

Managed Objuxts

MIB

ASN . 1

Object Identifier

ATTRIBUTES

(atomic)

NAME ATTRIBUTES

SET of OPERATIONS

NOTIFICATIONS (Classes)

EVENTS

INHERITANCE

CMIP ( rich, chaining )

CMIP (Connection-Oriented)

COMPREHENSIVE

DEVELOPING
SNMP

RFC 1155 [SMI]

Object-Type ( subset of GDMO )

MIB

ASN. 1

Object Identifier

VARIABLES

( sufifix of object-type )

N/A (explicit )

SET of OPERATIONS

TRAPS

N/A

N/A

SNMP ( subset , atomic )

SNMP ( Connectionless-Oriented )

SIMPLE

IMPLEMENTED

Figure 17 Comparing OSI and DARPA 


\section{CHAPTER 5 Fault Management}

The standards themselves only dictate methods of defining and communicating network management information. Being able to use that information to perform fault management is a key part of the network management problem. A survey of over 100 users by the OSINM Forum, found that "Clear and away the most critical need is fault management, especially the alarm reporting and logging functions," [1]. In today's world of distributed processing, the proliferation of workstations and LANs interconnected via intelligent bridges and routers makes the problem of fault management a very difficult one.

\subsection{The Fault Management (FM) SMFA}

The Fault Management SMFA [55] addresses the requirements and functions for the management of faults from the perspective of the user of network management. The aforementioned survey [1] reported that users "have commented that fault isolation is absolutely necessary." The Management Framework [37] describes fault management as encompassing:

"fault detection, isolation and the correction of abnormal operation of the OSI

Environment. Faults cause open systems to fail to meet their operational

objectives and they may be persistent or transient. Faults manifest themselves

as particular events (e.g. errors) in the operation of an open system. Error

event detection provides a capability to recognize faults. Fault management 
includes functions: to maintain and examine error logs, accept and act upon

error detection notifications, trace and identify faults, carry out sequences of diagnostic tests and correct faults."

\subsubsection{Fault Management Requirements}

The FM SMFA [55] identifies requirements and functions for fault detection and diagnosis in an open system, but does not identify functions for the correction of fault conditions.

\section{Fault Detection}

Cause and Effect: If a routine (system) should cease to change in its customary (designed) way, the break in the routine would form a change that would be detected. Such a change would impress us as an effect, and one would inevitably be driven to seek for a cause i.e. to ask why? Causes are certainly connected by effects; but this is because our theories (hypothesis) connect them, not because the world is held together by cosmic glue.

\section{Fault Diagnosis}

The mechanisms of diagnosis are not unique to diagnosis but can be subsumed as special cases of the general human mechanisms of problem solving. The mechanisms of diagnostic reasoning are a collection of information processing strategies appropriate to the knowledge of a particular domain. The domain of fault diagnosis is the 'why?' of a malfunctioning world (network) created by us. 
Diagnosis should be viewed as a set of basic tasks used in many situations in everyday life. Diagnosis is the process in which one must determine those components of a system which, when assumed to be functioning abnormally, will explain the discrepancy between the observed and correct system behaviour. The goals of diagnosis are:

- to identify the structural cause of the observed undesirable behaviour

- to explain how a particular undesirable behaviour came about (i.e. requires understanding)

There are several complicating factors in diagnosis. In general, it is possible to have multiple malfunctions. Often processes start with only a small number of observations and therefore one must apply an iterative process of tentative partial diagnosis and effective testing. Observations/sensors may be unreliable themselves and therefore, diagnosis and sensor validation need to work in cooperation.

\section{Fault Correction}

After determining that a problem exists, the next step is fault correction. There are two aspects: in the short term, the network manager must find a way around the problem; in the long term, the failed component must be repaired or replaced. Some network management systems incorporate a fallback or redundancy capability, which can be either automatic or require operator interveritiva. As of now, the OSI standards do not identify functions for the correction of fault conditions.

\subsubsection{Fault Management Functions}

The Systems Management Overview [42] states: 
"The concept of user oriented requirements is intended to provide a frame of reference within which requirements for functions may be identified. Each requirement is satisfied by one or more functions."

The FM SMFA [55] defines the following functions based on the use of CMIS [38] and the concepts defined by SMI ( [50], [51] and [52] ) :

- alarm reporting (ARF) [46]

- confidence and diagnostic testing (CDTC) [62]

- event reporting (ERF) [47]

\subsection{A Third Generation Network Environment}

The third generation of computer networking is characterized by LAN and clientserver technologies linking thousands of desktop PC's and workstations into cooperative computing groups. In turn, servers act as access points into the enterprise backbone network (WAN) which can extend geographically around the world. Generally, universities do not have access to very large, international, multi-protocol networks supporting mission critical applications. Therefore, it is both necessary and beneficial for universities doing research to collaborate with industry which do have these environments and the resulting fault management problems.

Figure 18 is a representation of the network environment that was used as the first example network for the PEGASUS Network Fault Diagnosis and Management Project. This represents a small portion of an organizations's extensive multinational network which spans four continents, connecting thousands of network stations through local and 
wide area links. The UNIX operating system environment supports TCP/IP, Ethernet, AppleTalk, NFS, DECnet (Digital) and XNS (Xerox) protocols. This company has almost total peer-to-peer network access for its employees who are involved in software development and engineering research and development. "At one site, for example, several thousand engineering workstations are connected in more than 200 workgroups bridged to more than 20 Ethernet backbones. Another 200 bridged Appletalk networks support some 2,400 Macintosh nodes." [77]

NETWORK ELEMENTS

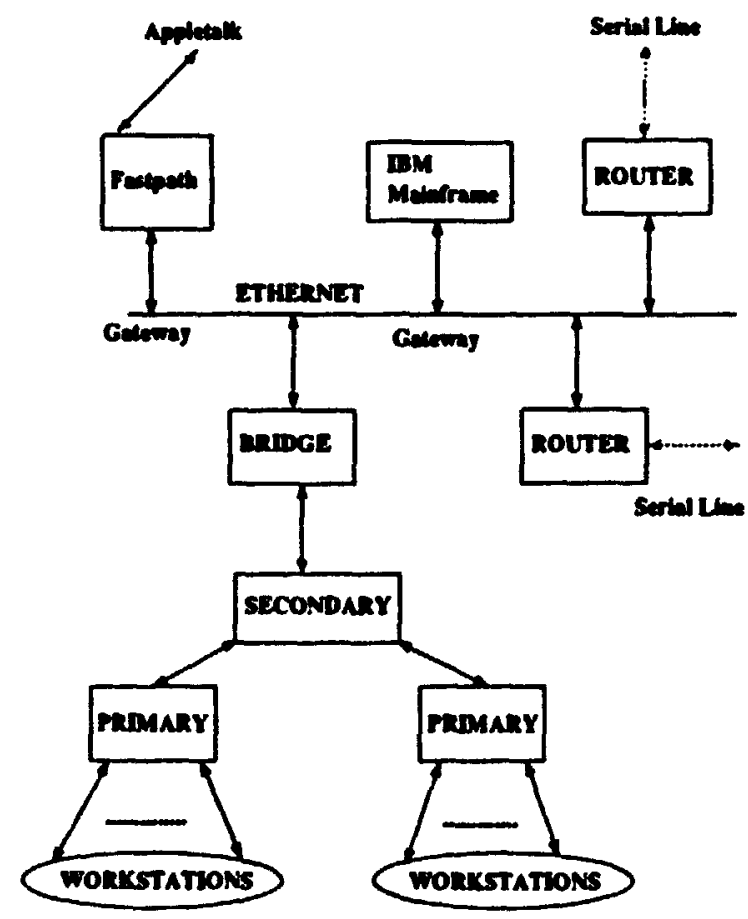

Figure 18 Sample Network Environment 


\section{HUBS}

Workstations are interconnected to Ethernet LANs via fanouts (hubs). A hub is a high-density, modular, multiport repeater that operates at the physical (layer 1) level. Hubs (secondary and primary, see Figure 18) are interconnected to form a pyramid configuration, through which all packets from the workstation's are transmined up to a bridge. Up to 16 workstations may be connected to eact: primary, and up to 16 primaries can be connected to each secondary concentrator. All major network resources, such as bridges, routers and mainframe gateways are connected directly to hubs.

\section{BRIDGES}

A bridge functions at the MAC sublayer of the OSI model's Data Link layer (layer 2) and is transparent to high-level protocols. Bridges can connect either local or remote networks and are capable of some isolation of network segments. 3ringes use a process of learning, filtering and forwarding to keep traffic within the physical segment it belongs in. Intrabridge communication allows workstations to communicate below a bridge without placing traffic on the Ethemet. Traffic addressed to workstations not below a bridge is transmitted onto the Ethemet and packets addressed to workstations below the bridge is accepted from the Ethernet (see Figure 18). 


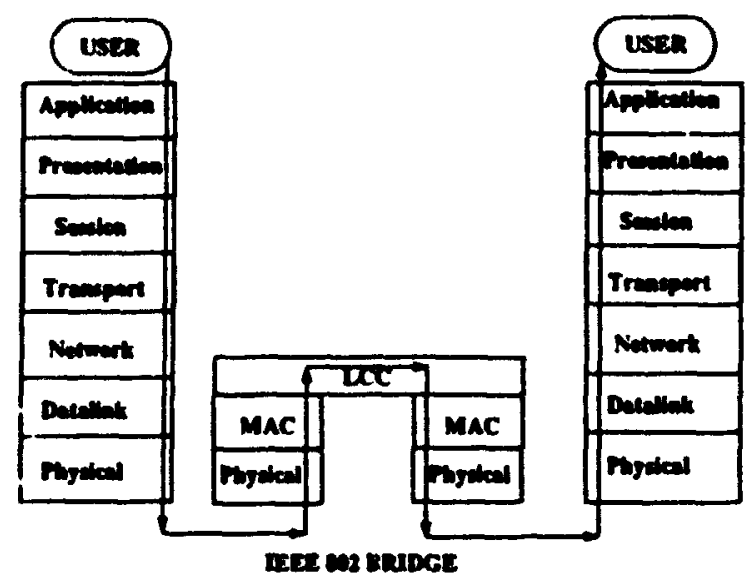

Figure 19 Bridge Functionality

Bridges cannot share information as they do not have access to the OSI network layer protocol. Thus, when a bridge gets overloaded, the other bridges will never know. Generally about five bridges are connected onto a single Ethemet. Figure 19 illustrates the functionality of a bridge in terms of the OSI Reference Model.

\section{ROUTERS}

Routers are designed to create a WAN from an intemetwork of LANs. A router functions at the Network layer (IP) of the OSI model to forward packets in accordance with the methods described by higher-level protocols. Routers can connect either local or remote networks and can create many logically different subnetworks within an internetwork. Routers make decisions that determine the most efficient data path between two network segments thus helping to control traffic fiow. They do not view the network from end to end, but only know where the next router (hop) is. Traffic between Ethernets is handled by routers interconnected by serial links (see Figure 18). 
Each router is typically connected to two Ethernets and to three serial lines. These lines range from $56 \mathrm{~Kb}$ to $\mathrm{T} 1(1.544 \mathrm{Mb})$, with the trend being steadily towards more and more T1s. Each Ethernet is configured with two routers for redundancy purposes and the maximum dimension of the network is greater than seventeen hops (Australia). Routers share information over the network by a proprietary control protocol. Multiple routing options are almost universal throughout the network. The dynamic capabilities of routers coupled with the multiple paths over an extensive network dimension makes management of this internet very complex. Figure 20 illustrates the functionality of a router in terms of the OSI Reference Model.

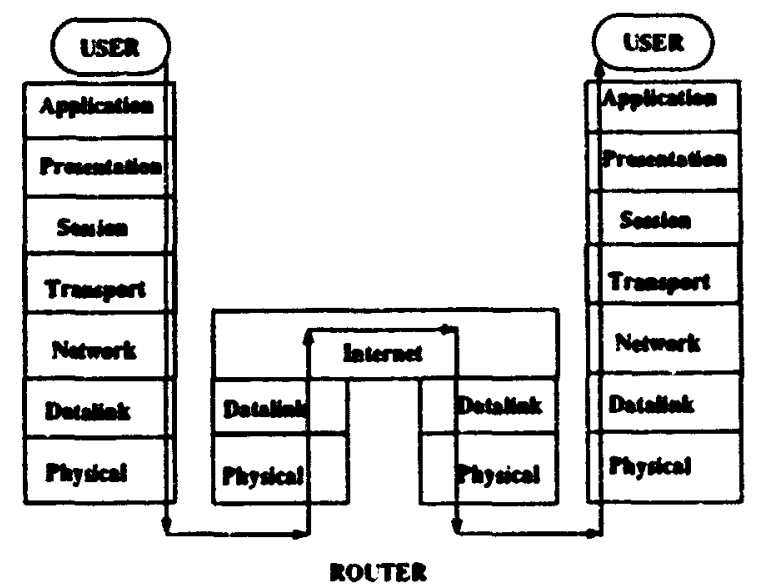

Figure 20 Router Functionality 


\section{GATEW'AYS}

Gateways operate on the top three layers of the OSI model (session, presentation and application). They are used to interconnect systems built on totally different communication architectures. Because there is no commonality, a gateway must translate all the data passing between the two systems. Gateways connect the corporate IBM mainframes to an Ethernet allowing the distributed workstations access to their applications and services (see Figure 18).

The organization has specialized work groups with different requirements. Gateways accommodate file transfers between Ethernet and Appletalk workstations. Appletalk protocols for the Macintosh workstations are accommudated on the Ethernet by piggybacking. Piggybacking means that the complete Appletalk protocol is carried in the data portion of the Ethernet packets. Fastpath gateway devices perform two-way mapping between Appletalk and Ethemet addresses (see Figure 18), as well as this piggybacking function. Another advantage of the gateway is that only traffic from a single gateway needs to be monitored, rather than each Appletalk workstation. Figure 21 illustrates the functionality of a gateway in terms of the OSI Reference Model. 


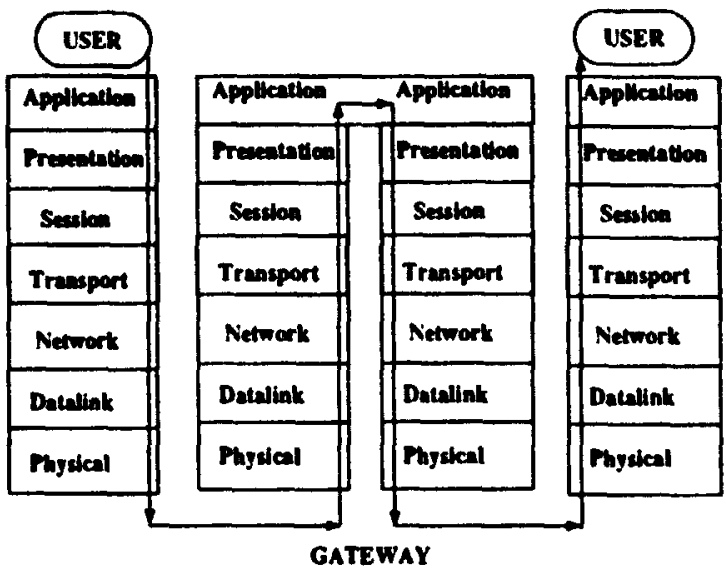

Figure 21 Gateway Functionality

There are approximately 400 Ethernet backbones or router-router serial links. The current network has approximately $i 400$ elements with a projection of $3-4000$ by the end of 1991.

THE FAULT MANAGEMENT ENVIRONMENT Figure 22 shows the current fault management environment that PEGASUS is addressing. The top of the diagram represents the central location for surveillance and managing of the entire network by software assisted human operators. Custom in-house developed interfaces are shown with broken lines connecting the label "Custom". SNMP-based interiaces are shown with broken lines connecting the label "SNMP". 


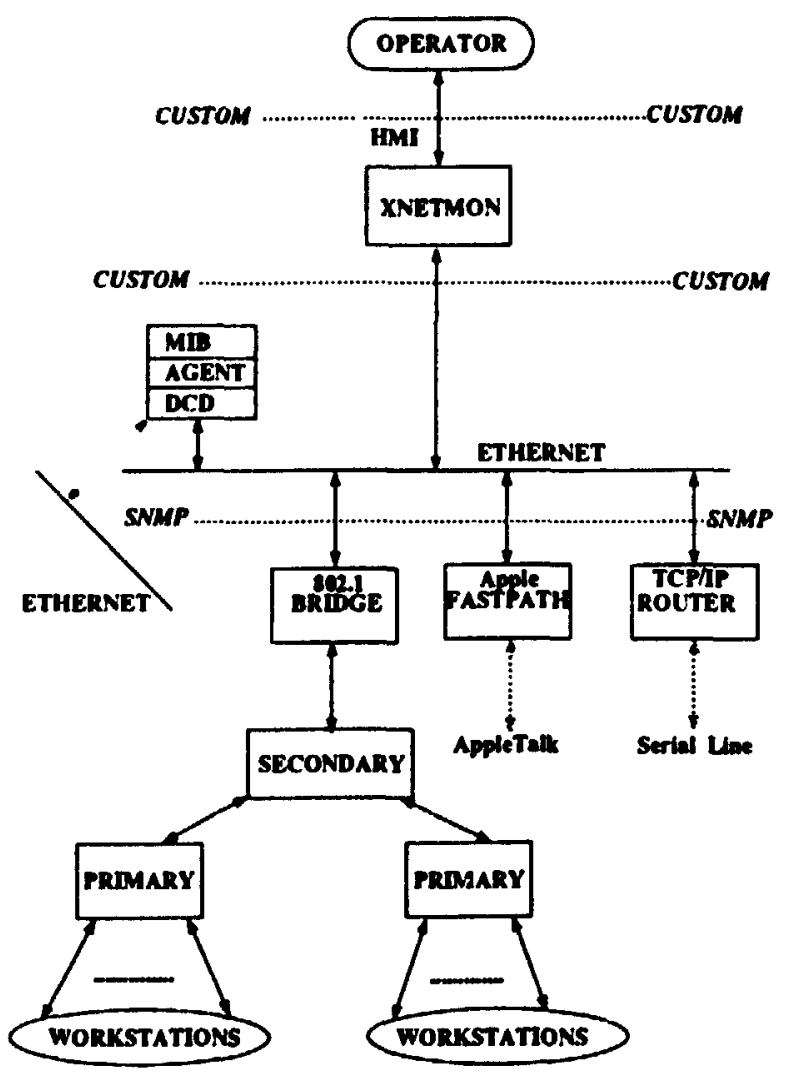

Figure 22 Sample Fault Management Environment

A network management station is a network element that has the functionality to communicate with network agents and that provides a user interface to the agents allowing users to view network agent data and/or to control network agents and thus the network elements. The network management station (XNETMON) is an inhouse customized implementation written in C+r. XNETMON is a icon-based, graphical interface network management station for displaying the status of network components. XNETMON is only a monitoring device and the diagnostic functions are performed by humans. 
XNETMON combines a topological representation of the network, based on the known network configuration, and status data collected and analyzed by remote data collectors (DCD). A data collector executes on a UNIX workstation on the network, which also executes a SNMP agent and its MIB, as well as proxy agents that invoke SNMP or PING. SNMP monitors key network elements and provides failure alarms. PING is a UNIX process which utilizes the ICMP (TCP/IP) protocol's mandatory ECHO_REQUEST datagram to elicit an ICMP ECHO_RESPONSE from a specified host or network gateway[81].

There are currently four independent data collectors filtering network status to XNETMON via a inhouse application layer protocol. The data collectors utilize proxies through the SNMP agent to poll the bridges, routers, Fastpath gateways and mission critical workstations that are generally servers. Devices that cannot support a certain protocol are managed through nodes (proxies) that agree to relay management requests to the lesser device using the device supportec protocol(s). The proxies translate to/from SNMP and the particular device proprietary protocol. The proxy collects in/out data counters and in/out error counters fur the network interface layer (layer 2) of the device for recording in the agent's MIB. Because only traffic from the Fastpath gateways is monitored, the status of individual Appletalk workstations is not made available to XNETMON. Asynchronous fault alerts (events) from the devices are communicated to the proxy for relay to the agent, to the data collector and subsequently to XNETMON.

A major design criterion for network management was the requirement to confine this status traffic to $1.5 \%$ of network capacity. Therefore, there is one proxy per bridge 
which polls every three minutes, while routers are polled every minute and servers are polled at five minute intervals. This was necessary because the SNMP protocol is "in band overhead" in contrast to the "production" network traffic. "A new project is being defined to gather baseline network statistics, measure the performance of applications and set standards,"[77].

Because XNETMON is a monitor, it can indicate problems (or symptoms), but it does not determine (diagnose) the why of the situation. The challenge is to diagnose and resolve any network difficulty proactively within the network. The increasingly important and difficult task is to ensure reliable, responsive network services in a dynamic, distributed environment. The problem is that the network is growing rapidly, requiring a direct increase in the number of human operators (cost) needed to manage it. More automated intelligence capability (e.g. PEGASUS) to augment the operators is needed. This would result in a more independent relationship between the size of the network and the cost to manage it.

Defining the Problem The following questions must be answered:

- How well does the tool scale up from a private industrial environment (above) to a global, public service provider such as the carriers provide?

- What is desired from a tool that helps network managers to understand the behaviour of protocols and the applications that use them ?

- What are the various skills required to achieve the desired results ? 
- How can network managers deal effectively with the complexity that is inherent in the solution space?

Desired Results The application user is most interested in the macroscopic behaviour of a specific application. The generic question for all users is "Am I receiving adequate performance from the transport protocol ?" Adequacy may be defined differently for the different applications-throughput, delay, availability, or some combination. In some cases (e.g. new versions of protocol implementations), it may require verifying proper protocol operation. From the perspective of the transport layer, the network layer and/or lower layers may not be performing well. From all viewpoints, the default desire is "Tell me everything you can about the input data, most interesting first." The ability to answer such questions by pointing out possible causes and by recommending possible solutions is the goal of the PEGASUS Network Fault Diagnosis and Management Project [21].

To achieve this result, we imagine supplying various types of data to a black box and receiving diagnoses and recommendations as output. This basic scenario can be made more complex by the addition of external feedback loops and through incremental addition of data (e.g. by querying the user as data is needed or from other black boxes working on the same problem). The input data consists of facts about the environment (such as topology, configuration parameters, network management information) and observations (such as "connection still working, but noticeably slower"). The black box starts grinding away, perhaps asking questions occasionally, and methodically searches the solution space, guided by both initial and ongoing input data. The use of automatic monitoring tools allows problem resolution to begin at the earliest possible time, possibly before it 
has been noticed by the user. However, some problems will always occur that monitoring tools cannot identify, and users provide the fault detection role. Some problems exhibit no obvious user-visible symptoms, such as degradation of service and redundant link failure.

\subsection{The Fault Management Solution}

"Eventually, implementations of network management systems must incorporate

highly interwoven principles from the frontiers of distributed processing.

non-linear feedback control, security and artificial intelligence." [6]

The standards work does not define "the applications that must provide the seamiess view, end-to-end management, and top-down control necessary for actual integrated network management of multi-vendor heterogeneous networks," [96]. The question is how to apply these standards in a fault management application to perform in a real third generation computing network environment. A major issue is the need to identify the boundary up to which Management Standardization of objects is done generically and beyond which resource based definitions of objects takes over.

\subsubsection{Previous Fault Management Approaches}

Appendix $\mathrm{C}$ contains a bibliography of some previous approaches to fault management by others in earlier research work.

\subsubsection{PEGASUS}

PEGASUS [21] is a multi-module r:search system attempting to automate the diagnosis process to find faults in communications networks. It is part of the "Fault Management 
in Communications Networks" project of the Department of Systems and Computer Engineering, Carleton University supported by the Telecommunications Research Institute of Ontario (TRIO). PEGASUS is in the preliminary architectural design phase but will be prototyped using C++ on a Sun Sparc workstation in a SunOs (UNDX) TCP/IP network environment. Areas of research will include the extension of a static network description to include dynamic elements and near real-time behaviour. Diagnostic problem classes to be researched vary from single component failures (e.g. server or modem), to relationship failures (e.g. terminal-modem or file transfer between a server and a workstation), to two and three node failures (e.g. complex router problems due to the designed redundancy in the network). Future emphasis will be placed on orientation towards the OSI network management framework.

\subsubsection{Applying OSI STANDARDS to NETWORK MANAGEMENT (NM)}

Status of OSI Network Management Standards and Implementors Agreements As discussed earlier, the Open Systems Interconnection (OSI) management standards, while currently at an intermediate stage of their development, are maturing rapidly. The ultimate goal of these standards is to enable the development of interoperable. multivendor products for the management of computer and communications systems and networks. Key areas of management standardization are architecture, protocols, systems management functions, and the structure of management information. The Common Management Information Services and Protocol (CMIS/P) standards have now become International Standards (IS). Many other needed management standards are still at the 
Draft International Standard (DIS) status. However, these DISs, available at the beginning of 1991, compose a subset of management standards that make it possible for vendors to build useful systems to meet some immediate network management requirements.

Another important aspect of network management standards activity is the development of international Implementation Agreements (IAs). The Network Management Special Interest Group (NMSIG) of the OSI Implementors' Workshop (OIW) (sponsored by NIST and the IEEE Computer Society) is developing IAs based on the emerging OSI Network Management standards. These agreements are being developed in phases that align with the ISO/IEC standards as they progress from CD (Committee Draft) to IS. The OIW Network Management Phase 1 IAs became stable in December 1990 [78].

\section{U. S. Government Network Management Profile (GNMP) [80]}

In the absence, at present, of a complete set of mature standards for network management, a set of initial network management specifications that contains a useful subset of the planned system and network management functionality provides an interim solution to meet some high priority requirements. It is proposed by the U.S. NIST that an integrated network management system can be developed in phases.

The U. S Government Network Management Profile (GNMP) [80] is the standard reference for all U. S. Federal Government agencies to use when acquiring Network Management (NM) functions and services for computer and communications systems and networks. Version 1 (May 1991) of GNMP specifies an initial, useful set of standards to permit an network manager to issue unambiguous procurement requests for standard, 
interoperable network management products capable of operating over networks using standard protocols.

The U.S. Government Open Systems Interconnection Profile (GOSIP) is cited in the GNMP to specify the protocol stack upon which management information can be conveyed. The GOSIP also specifies services, such as File Transfer, Access and Management (FTAM), Message Handling System (MHS), and Virtual Terminal (VT), that can be used to support network management applications. Future versions of the GNMP will enable management of more GOSIP components (e.g., transport connections and key exchanges.) Future versions of the GOSIP will cite the GNMP to specify the management protocols, services, and information needed to facilitate interoperable multivendor management of GOSIP-compliant systems. As both the GNMP and the GOSIP mature, it is expected that they will continue to cross reference the latest versions of each other.

Scope of GNMP The GNMP specifies the common management information exchange protocol and services, specific management functions and services, and the syntax and semantics of the management information required to support monitoring and control of network and system components and their resources. Version 1 of GNMP specifies:

- 1) the Common Management Information Services (CMIS)

- 2) the Common Management In:ormation Protocol (CMIP)

- 3) the following five systems management functions (SMFs). 

a. Object Management Function (OMF)
b. State Management Function (SMF)
c. Attributes for Representing Relationships (ARR)
d. Alarm Reporting Management Function (ARF)
e. Event Report Function (ERF)

- 4) the Structure of Management Information (SMI)

Additional management capabilities and managed objects can be included in subsequent releases of the system. Eventually, as the OSI network management standards all reach technical maturity, the GNMP will embrace the full set of management functionality.

\section{Version 1 of GNMP}

Version 1 of GNMP includes OSI Common Management Information Protocol (CMIP) and five general management support functions. More management support functions will be included in future phases as these functions become available. Version 1 of GNMP incorporates managed object definitions for network interfaces, including LANS, X.25, Integrated Services Digital Networks (ISDN), Fiber Distributed Data Interface (FDDI), modems, bridges, and links. Additional managed objects are planned in subsequent phases. The eventual scope of the GNMP will be extended to include system management objects for applications, services, operating systems, and database systems.

The GNMP builds on the OSI lower (layer 1-6) protocols to provide basic interoperability in support of CMIP. In addition, three OSI (layer 7 ) applications that might be 
useful in a general purpose network management solution. The File Transfer. Access. and Management (FTAM) application facilitates the transfer of bulk information, such as routing tables, billing records, audit trails, and configuration data. The Virtual Terminal (VT) application enables remote login to network management systems; thus, allowing a network operator to execute proprietary network management tools and diagnostics. The Message Handling System (MHS) permits network operators to exchange messages with each other while attempting to diagnose or correct network problems. MHS can also be employed to alert users about network status changes. Future phases of the INM will include managed object definitions for layers 3 through 7 for OSI end systems and for layer 3 for OSI-compliant intermediate systems.

Taken together, these network management standards define aspects of network management that must be implemented in a standard way to allow interoperable, multivendor management. Figure 23 illustrates how these management standards fit in the application layer stricture in an open system. The application layer standards, such as FTAM, that may be used by network management applications are included as well and are designated as shaded optional elements.

Those non-standardized, but generally required, network management elements, such as the human-machine interface (HMI), database management system (DBMS) and analysis functions are designated in the illustration (as dashed figures) as recommended elements. These elements are the sub-domains of the XNETMON and PEGASUS implementations. Future research directions in these areas are proposed for PEGASUS in Chapter 6 (6.2) of this thesis. 


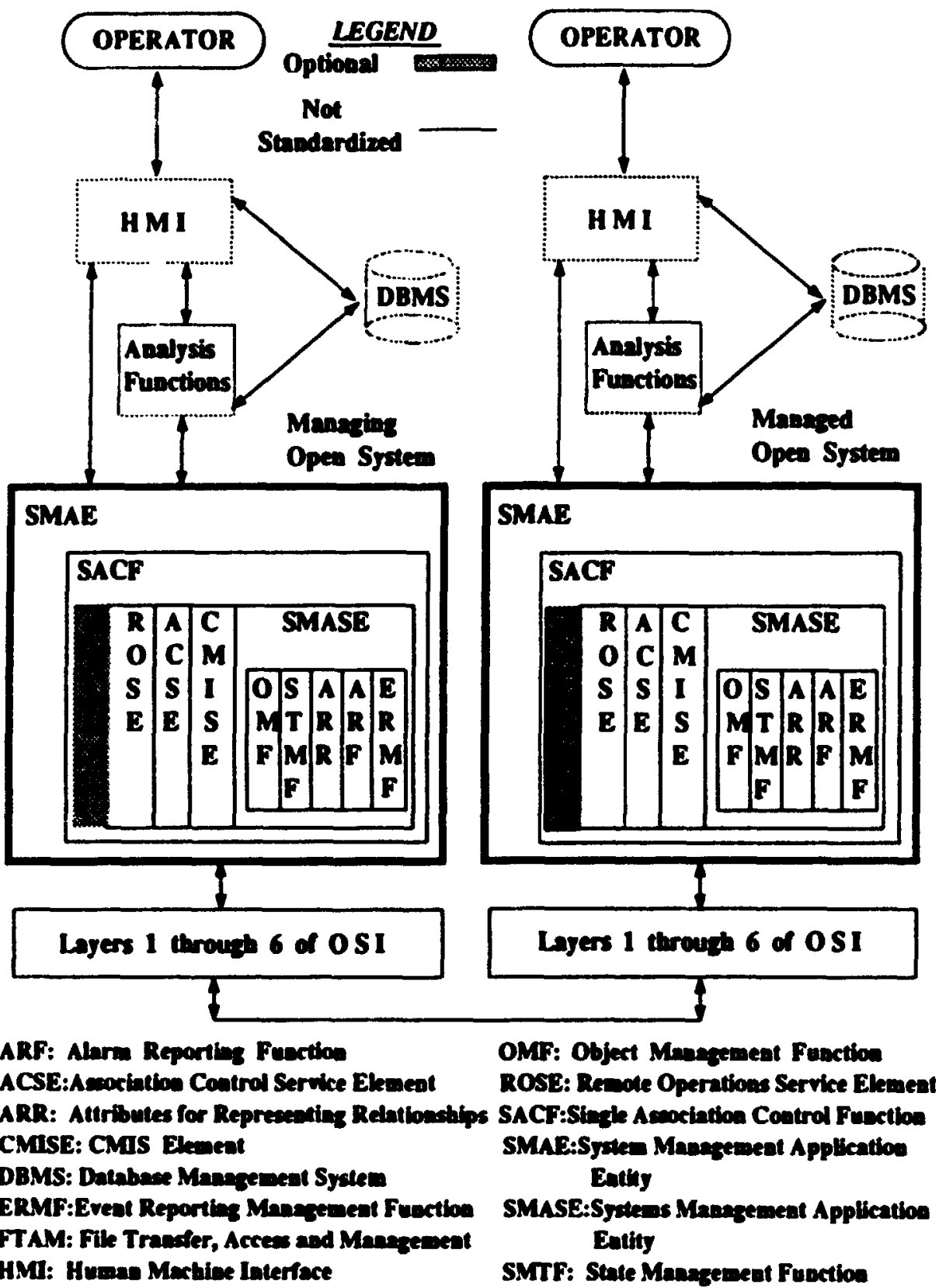

Figure 23 Components of Interoperable Management Open Systems 
Management Information The ma -gement of local area networks (LANs), as well as the bridges that interconnect them, represents a key network management requirement. An obvious need is the requirement for management information definitions. As discussed previously, some support management information has been defined by the ISO/IEC Network Management working group and they have specified a set of guidelines and templates for defining management information, they have not specified the definitions of particular management information to be monitored and controlled. Various standards-making groups and implementors forums are currently developing definitions of management information pertinent to their specific areas of expertise and standardization by using the standard definition templates that ISO/IEC has developed.

Defining management information not only requires in-depth knowledge of the specific subject areas to which the management information belongs, but also requires understanding of the management information model and the standard templates. Consequently, it takes extended development time to define specific management information. In accordance with the prioritized network management requirements (i.e. LANs), Version 1 of the GNMP focuses, mainly, on definitions of management information pertaining to implementations that perform OSI layer 1 and 2 functions. To achieve uniformity in the definition of management information, thus aiding the development of au'omated tools, such management information should be defined using the techniques and templates as specified in GDMO.

Interim OSI Network Management (INM) For the example organization employing the SNMP-based network monitoring system illustrated in Figure 22, an application of 
the GNMP (see above) and its Manager of Managers (MOM) approach (sec Chapter 4, Conclusions) is proposed by this thesis. This proposal is illustrated in Figure 24. This represents a generalized Interim OSI NMsolution (INM). This diagram assumes that some conversion of central SNMP-supported hubs and bridges to OSI-supported hubs and bridges has taken place. This allows for a phased conversion to OSI/CMIP. XNETMON is replaced by a Corporate Management Integrator. The number of DCDs is deceased or are no longer needed. All Apple FASTPATH and TCP/IP routers remain in place, using SNMP means to manage these unconverted resources.

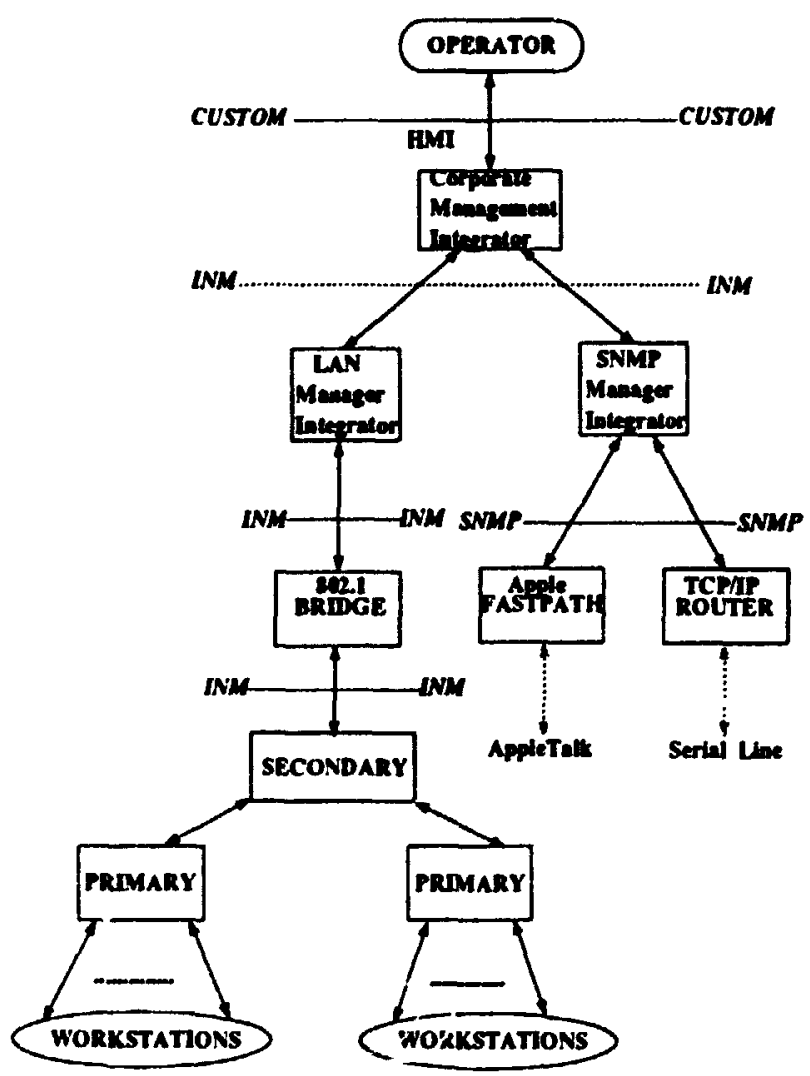

Figure 24 Interim Network Management (INM) using CMIP 
Three network maliagement integrators have been added: one (LAN Manager Integrator) integrates the OSI LAN and bridge information, one (SNMP Manager Integrator) integrates SNMP information, and one (Corporate Manager Integrator) integrates the LAN Manager and SNMP Manager Integrators. Implementation of such a hierarchical network management system requires a standard for network management information exchange between integrators, and between integrators and managers. Ir Figure 24, interfaces using OSI network management standards are shown with br.,ken lines connecting the label "INM". SNMP-based interfaces are shown with broken lines connecting the label "SNMP". Custom in-house developed interfaces are shown with broken lines connecting the label "Custom". The latter are the domain of XNETMON and PEGASUS implementations.

The SNMP Manager Integrator acts as an element manager for the OSI-based corporate network manager. The SNMP manager exchanges SNMP protocol with the devices on the network and exchanges CMIP (through the CMIP agent) to the "enterprise" manager. However, the SNMP manager is now responsible for translating the SNMP object into the OSI object.

The Simple Network Management Protocol (SNMP) is :widely implemented and is likely to be deployed to manage routers. In such deployments, SNMP can be viewed, analogous to proprietary local area network management protocols, as an intemetwork management protocol (i.e., for managing sets of routers). Thus, the SNMP can be fitted into a network management architecture that also includes the OSI protocols. Providing such a capability (i.e., to integrate SNMP into a single OSI network management system) 
involves the dual stack approach (see Chapter 4, Conclusions) for the SNMP Manager integrator. Because of the differences in MIB structures, the SNMP Manager Integrator has to deal with the translation (semantics) of the two managed object types (OSI and SNMP). In other words, the SNMP Manager Integrator acts as a OSI proxy for all of the SNMP managed portions of the network not yet converted to OSI. Therefore, the management applications in or above the Corporate Manager Integrator only need to understand OSI objects.

The GNMP specities an OSI standards (CMIP) for the exchange of management information between integrators and between integrators and managers. The GNMP specification can also be used hetween managers and networic elements, should the network elements possess sufficient computing capability (CMIP). The scope of the proposed INM encompasses a set of protocols for multi-vendor communications, a set of general-purpose management functions, and a standard set of managed object definitions. This scope addresses only the exchange of management inf ormation in a standard way in order to achieve integration of management systems and components made independently by a variety of suppliers.

Fault Management and Other Issues Other important issues are outside the scope of the GNMP, and thus the INM. Consider, for example, the analysis of management information utilizing artificial intelligence techniques or expert systems. For any operational network management system, raw management data must be collected, stored, calculated, and correlated to provide useful outputs for network planning, fault prediction, and billing. Requirements in these areas are outside the scope of the GNMP and must 
continue to be specified directly by the policies of the human network manager. Humanmachine Interface (HMI) is also outside the standardization scope of the GNMP. The network manager must continue to specify any requirements regarding presentation and ease of use. The usual issues of configuration and sizing of network management components are also outside the scope of the GNMP. The network manager must continue to plan the deployment and sizing of specific integrators and managers in accordance with operational requirements. This application of the GNMP, then, addresses a single, significant network management integration problem: interoperability between network management components; but not how to perform fault management or to diagnose.

Conformance Testing and Interoperability Testing Currently, there are no conformance tests available for an GNMP implementation (i.e., OSI systems management functions and managed object classes). However, conformance testing for management communications (CMIP) is expected to be available soon.

Because of this lack, interoperability testing for the INM implementation of Version 1 of GNMP should be strongly considered. Possibilities for such testing include the use of commercially available interoperability testing services, OSINET, or on-site multivendor testing to assure interoperability.

\subsubsection{Applying OSI STANDARDS to the Representation of NETWORK ELEMENTS}

Generally, every communication resource that must be described in order that its behaviour can be monitored and influenced, must be modeled as an object. Version 1 
of the GNMP focuses, mainly, on definitions of management information pertaining to implementations that perform OSI layer 1 and 2 functions.

\begin{tabular}{|c|c|}
\hline MANAGED OUJECT & ATTRIDUTES \\
\hline birtive & 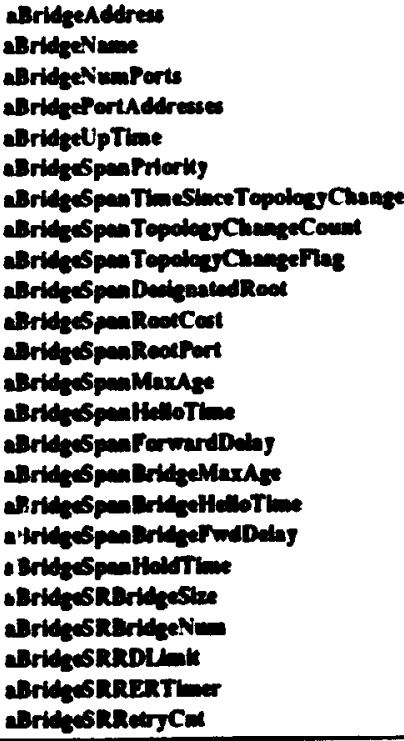 \\
\hline 5raballater & 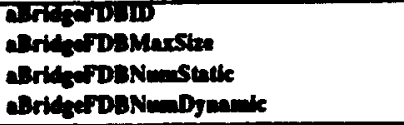 \\
\hline 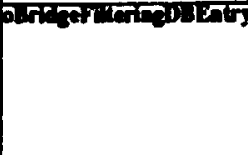 & 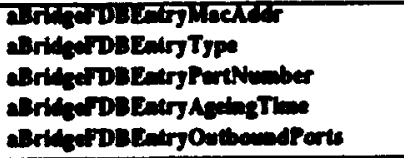 \\
\hline pirkerenter & 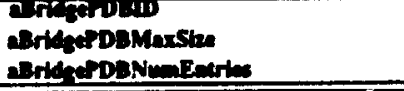 \\
\hline
\end{tabular}

Figure 25 IEEE 802 LAN Bridge Managed Objects

Bridges are ideal sites to collect general network layer 2 statistics such as percent network utilization over time and the number of collisions or alignment errors. In an effort to facilitate the integrated management of networks, the IEEE has begun developing a LAN bridge MIB (model) that can be consistently managed using different management 
protocols, without changes to the identification, registration, and semantics of the defined MIB elements. The IEEE 802 LAN bridge has seven managed object classes and 54 attribute classes which are illustrated in Figure 25 and Figure 26.

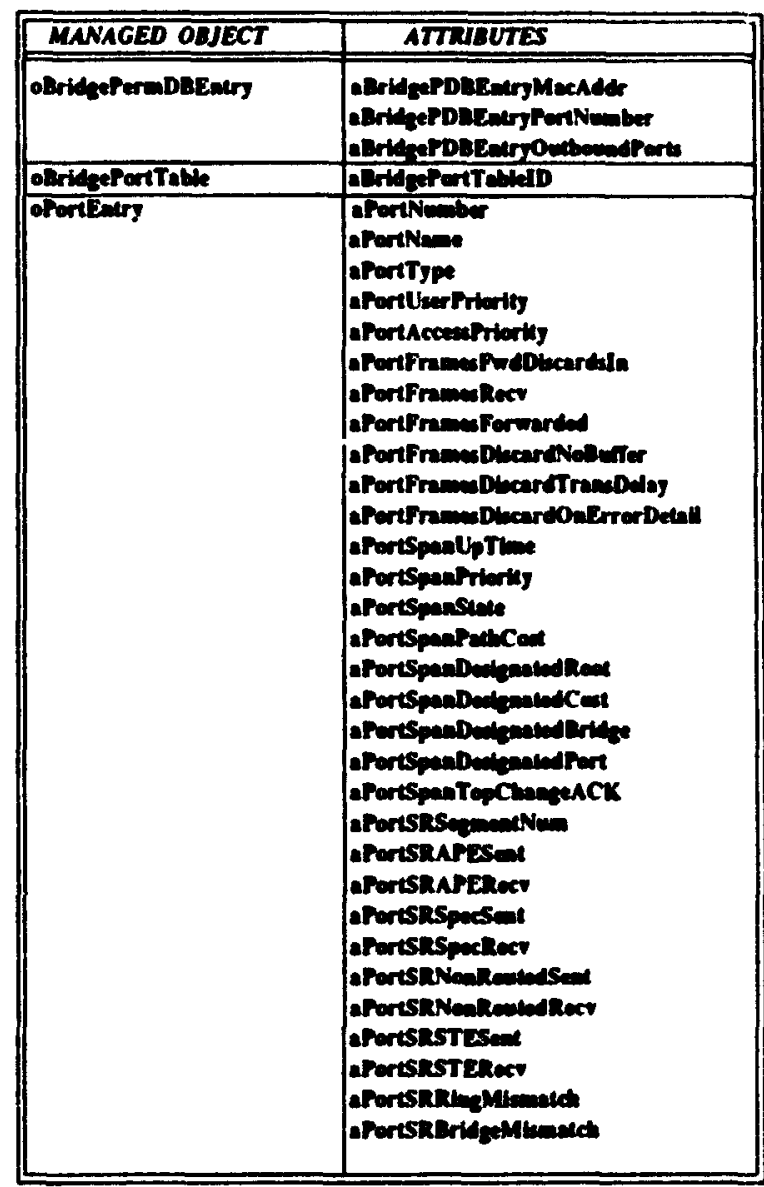

Figure 26 IEEE 802 LAN Bridge Managed Objects (cont.)

IEEE must specify their standard MIBs in the GDMO format for compatibility with the CMIP protocol. This LAN bridge MIB definition recognizes that IEEE $8(12$ LAN bridges will exist in many environments, including OSI environments, proprietary 
environments and portions of the TCP/IP internet which may be managed using SNMP. This LAN bridge definition is defined for consistency in both CMIP and SNMP. The IEEE goal of protocol independent managed object definitions minimizes the conversion development effort needed for the dual stack SNMP Manager Integrator in Figure 24.

Figure 27 is a schematic of the IEEE 802 LAN Bridge Name Bindings which shows the input from both ISO/IEC 10165-2 and RFC1213.

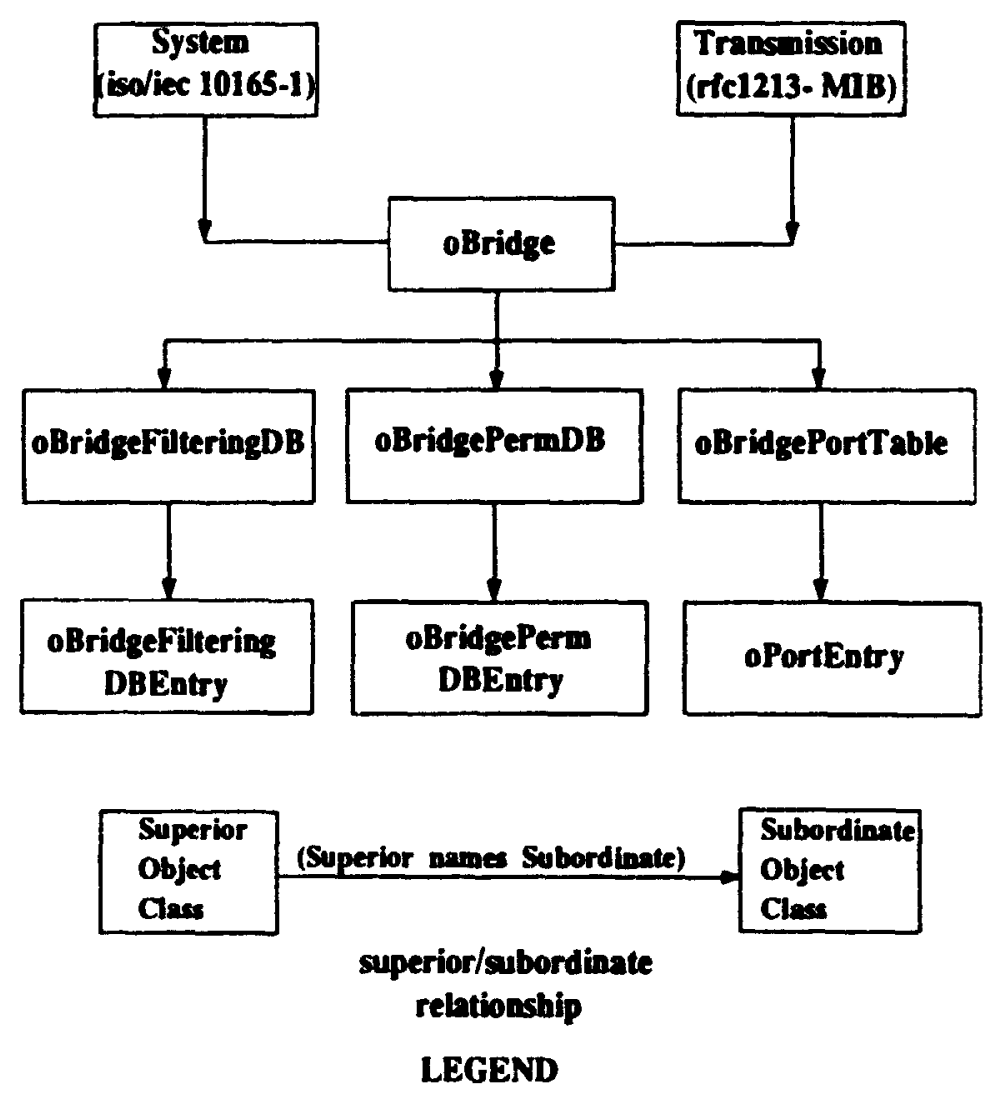

Figure 27 IEEE 802 LAN Bridge Name Bindings 


\section{Containment}

Containment relationships need to be explicitly identified for purposes of object addressing and naming. Figure 28 illustrates the containment of an IEEE 802.1 For example, oBridge is a superior object of oBridgePortlable which is a superior of oPortEntry which contains fourteen attribute classes, such as aPortNumber and aPortFramesDiscardOnErrorDetail.

\section{IEEE CO2.1 LAN BRIDGE}

- Heal sites to colliect generd betwort statstics

eg. - pereent wetwork millizition over thwe

- number af collitions or alleminent errans

\section{CONTAINMENT}

(le e e BridgeMOC 1)

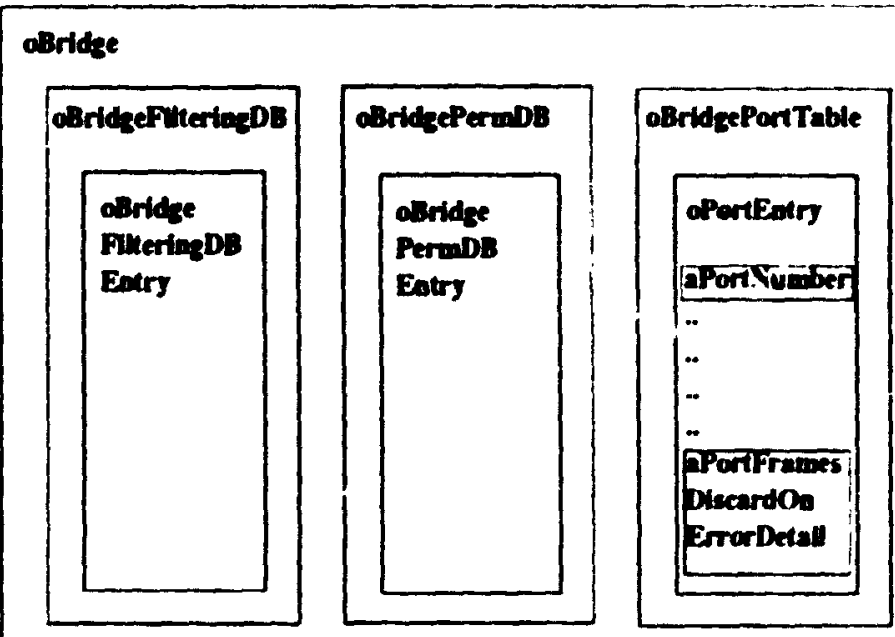

Figure 28 IEEE 802.1 Containment 
This containment hierarchy can be used for network management purposes to visualize the real world hierarchies of every IEEE 802.1 LAN bridge, no matter how they are physically designed and implemented. This is the purpose of the OSI SMI and GDMO standards.

Even though the design of object model for a network resource ( eg. Figure 28 ) can be directed by the standards, the transfer syntax for this model can still be uncertain due to different implementations. For instance, there is a choice of different programming languages for any software engineering project. Given the same language of choice (e.g. ASN.1), different programming styles and techniques can still defeat the purpose of a common representation. As a result, some protocol dependent syntactical differences potentially exist for transferring the data elements (e.g. BOOLEAN and BITSTRING variables when used with SNMP). But most importantly, the identification, registration, and semantics of the data elements must be preserved during the transfer of these data elements. The encoding of the elements during the transfer is up to the protocol and has no bearing on the information transferred as long as each side of the exchange agrees to the registered identification and semantics (i.e. MIB). Thus, though the GDMO templates include syntax in the registration, that syntax only applies to their use with ISO CMIP. Other management protocols are free to choose their own transfer syntax (i.e. SNMP) -including not using ASN.I at all, if so desired.

\section{Inheritance}

Figure 29 illustrates the class inheritance hierarchy of the IEEE 802.1 LAN bridge. The TOP class defined in DIS 10165-2 is the superclass of the hierarchy. The 7 managed 
object classes (prefix " 0 ") are all one level below TOP and inherit the five mandatory attributes from TOP as shown in Figure 13. The 54 attributes (prefix "a") are all two levels from TOP and inherit from the 64 bit counter class, COUNTER64. The annotations 1 and 2 on the left of the ASN.1 definition in Figure 29, delineate attribute 1 and atribute 12 of the 32 attributes from Figure 26 that are encapsulated in the oPortEntry managed object class. 


\section{IEEE 802.1 LAN BRIDGE}

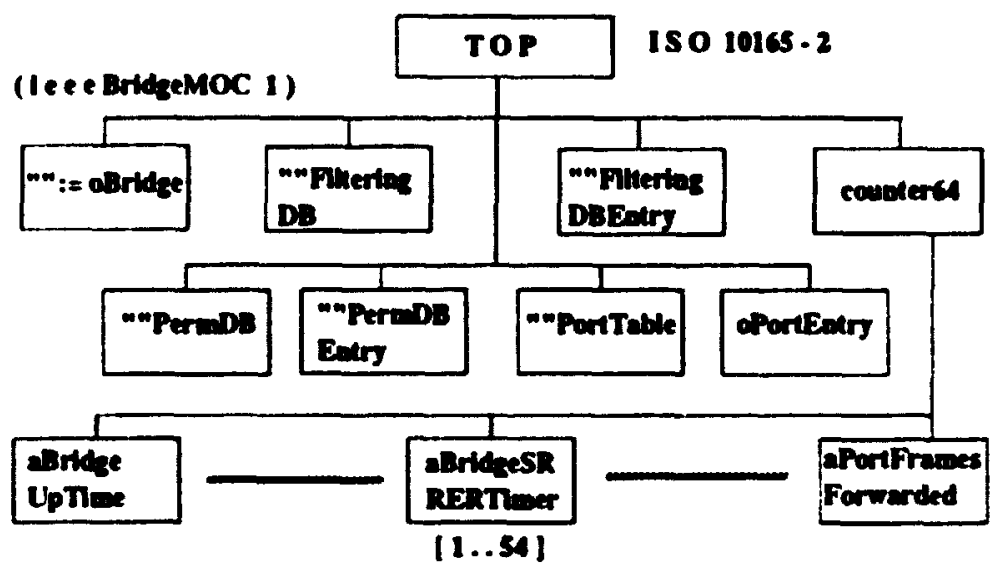

-7 MANAGDO ONJECTS ad SA ATTRDUTX

6. opontinary MANACED OHJDCT CLATS

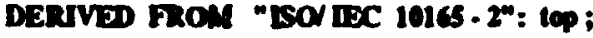
CanRACTERrad BY perideportindryite PACKACE DEAAVIOUR DEPINTIONS

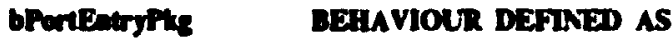
ne 82.10 , ctanses 661, 621 i; ATTRDUTRS

1 APortiumber GET, -. encoine autribute

2 12 attributes nos

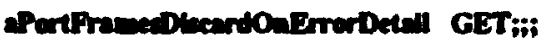
CONDTIONAL PACKAGES prontionyspantreatirs

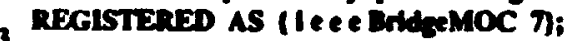

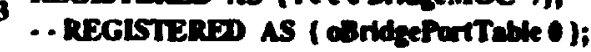

Figure 29 IEEE 802.1 Class Inheritance

Registration To accomplish this common MIB approach, two alternate conventions for assignment of OBJECT IDENTIFIERS are included in the MIB definition. As can be seen at annotation 3 in Figure 29, one line of REGISTERED AS \{ .. \} is an alternate replacement for the line just above it. The second line indicates the type of structuring of 
Object Identifiers (OIDs) required to facilitate manipulation with SNMP. or the syntactic changes necessary for conformance to the Internet SMI syntax restrictions. The resultin two object identifier sub-trees for registration are shown in Figure 30.

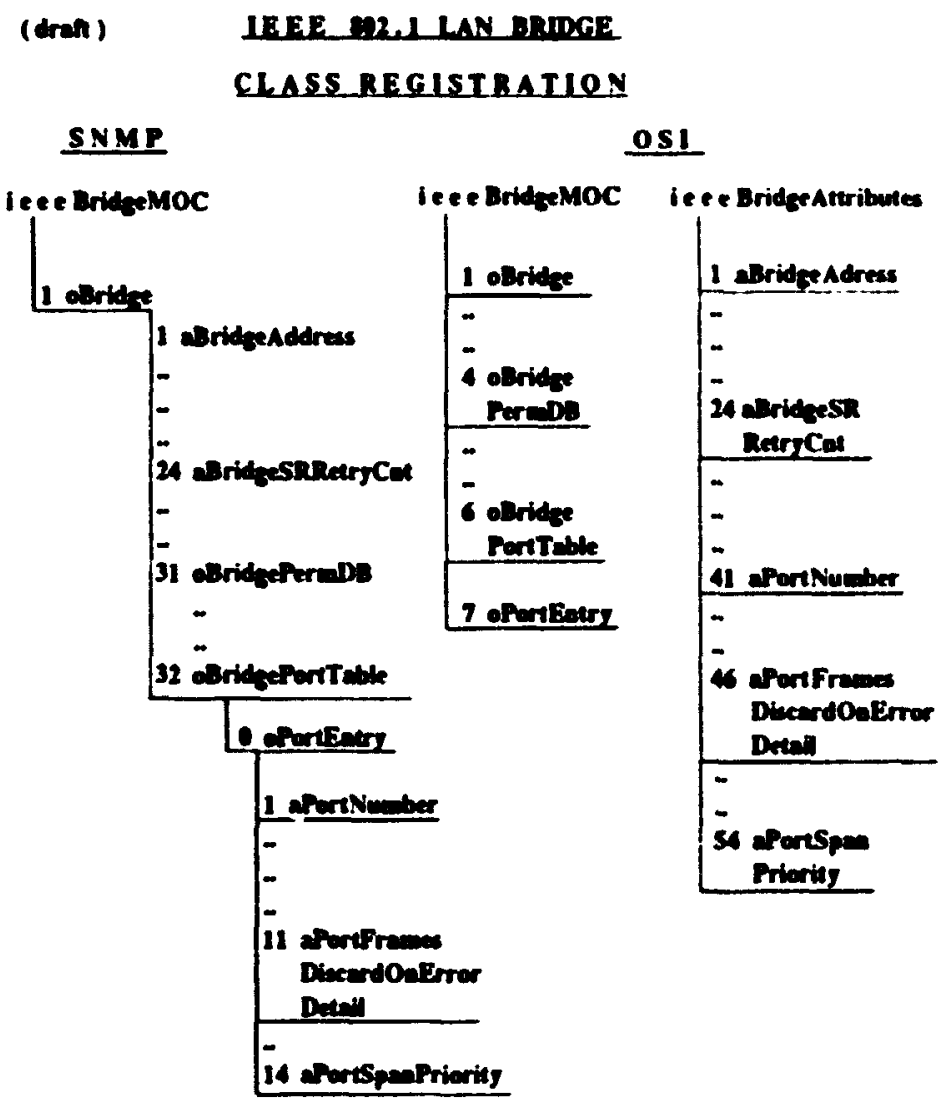

Figure 30 IEEE 802.1 Class Registration

Conversion rules are supplied to facilitate SNMP and dual stack implementations. For example, all BOOLEAN variables must be changed to INTEGER; all BITSTRING variables must be changed to OCTET STRING; and all attributes (except thuse whose only use is for naming in CMIP and those inherited from TOP (Figure 29)), become 
OBJECT-TYPES (see Figure 30). Therefore standards augmented by agreed to implementation constraints are required.

Conclusion In heterogeneous network environments, users are faced with disparate vendor-specific displays and command languages for each class of network component. To facilitate the integrated management of networks, managed objects that can be consistently managed using different management protocols, without changes to the identification, registration, and semantics of the defined MIB elements are needed.

The most reasonable solution to this problem is to ignore differences about protocols (e.g. SNMP and CMIP) and to encourage the individual MIB working groups to develop MIB definitions that support both the IETF SMI and ISO SMI. The example implementation of the IEEE goal of protocol independent managed object definitions, as illustrated by the above 802.1 LAN Bridge, should be encouraged and endorsed as a standard methodology, de facto or de jure.

This would ensure that all MIB definitions - which really just define manageable resources, without dependence on management protocols - were aligned across whatever management protocol or management system was used by an administrator for managing an environment. If this issue is not resolved, there is the risk of having different management definitions (MBs) for the same resources. This would waste resources both within the IETF and OSI, as well as within every vendor and many customers. 
Simple Systems-IEEE 802.3 hub standards

All major network resources, such as bridges, routers and mainframe gateways are connected directly to hubs (layer 1). Therefore, hubs provide an ideal focal point from which to observe and control the network at layer 1. In 1990. The IEEE 802.3 Working Committee formed a task force to develop standards for managing Ethernet network hubs [91].

The Hub Management Task Force focused its efforts on developing specifications for two types of management: managing the hub itself; managing network resources from the viewpoint of the hub. To avoic duplication of efforts, work was performed in cooperation with other standards bodies such as the IEEE 802.1, ISO and IETF.

The hub management specification will define how a hub can monitor network operation on a per-port basis and how managers can use it to control the resources attached to each network link. The $\mathbf{8 0 2 . 3}$ Hub has seven managed object classes and 44 attribute classes which are tabulated in Figure 31 and Figure 32. 


\begin{tabular}{|c|c|}
\hline MANAGED ODJECT & ATTRISUTES \\
\hline Hub & 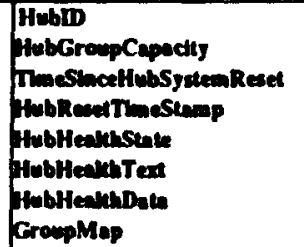 \\
\hline Resonercetypelo & 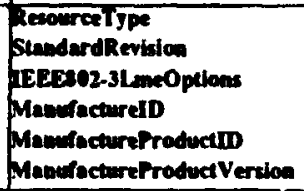 \\
\hline $\begin{array}{l}\text { Relay } \\
\text { Gaugelaitid Value }\end{array}$ & 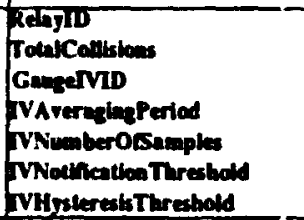 \\
\hline Group & $\begin{array}{l}\text { Growplo } \\
\text { Wumeroritans }\end{array}$ \\
\hline Pont & 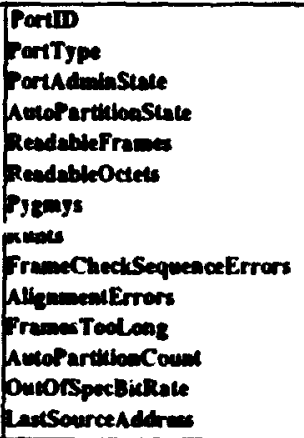 \\
\hline
\end{tabular}

Figure 31 IEEE 802.3 Hub Managed Objects 


\begin{tabular}{|c|c|}
\hline MA'AGED OEJECT & ATTRIDLTES \\
\hline Hab & 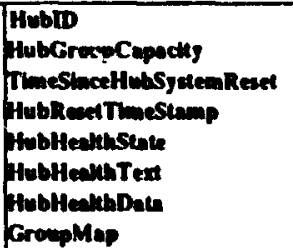 \\
\hline Resourcetijpelo & 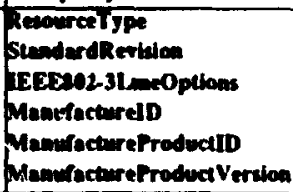 \\
\hline $\begin{array}{l}\text { Retay } \\
\text { GunelinitidValue }\end{array}$ & 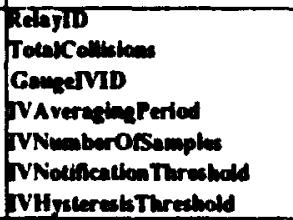 \\
\hline Growp & $\begin{array}{l}\text { GrowplD } \\
\text { Nenberorports }\end{array}$ \\
\hline Port & 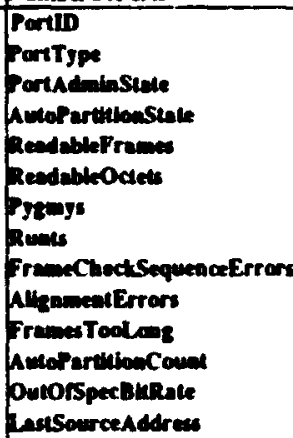 \\
\hline
\end{tabular}

\section{Figure 32 IEEE 802.3 Hub Managed Objects (cont.)}

The hub management specification's segment monitoring functions, based on the 802.3 Layer Management Standard, will examine network traffic, collect traffic statistics and map this information to the originating port These monitoring functions let the hub provide information on traffic volume as well as the frequency and type of errors originating on a particular segment. The hub management software will monitor the traffic from each segment without having to load additional management software into 
memory constrained workstations. This is a key advantage of hub management. The draft 802.3 hub specification for the Port Managed Object class is shown in Figure 33.

(drat) IEEE H2,1 MUD

- Leal sites to observe and contral

Fort Manged Object Class

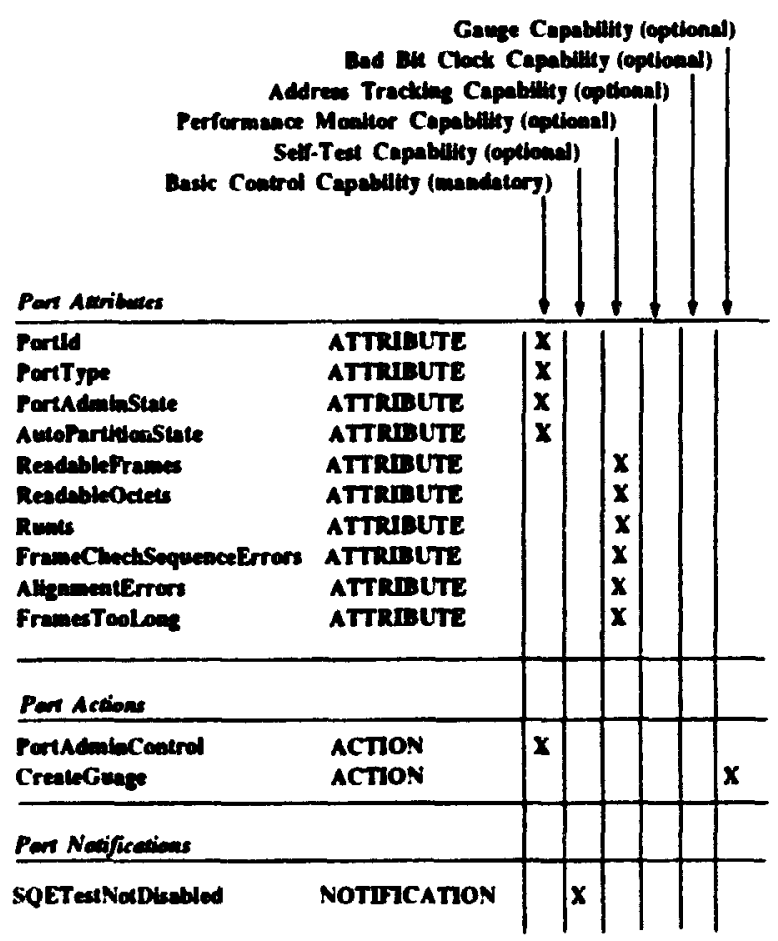

Figure 33 IEEE 802.3 Hub Port MO Class

To speed ISO acceptance of hub management, the draft document was written using the GDMO. The standard will be written to make it protocol independent. The proposed management standard can use either CMIP or the 802.1 management protocol to provide communication between network management stations and the hub. The 
Hub Management Task Force went to considerable lengths to ensure that the hub management standard will be able to accommodate the limitations of SNMP. To promote interoperability, the hub management standard makes generating the ASN.I encoding straightforward. It is expected that many implementations will use the managed 802.3 network to communicate with hubs, but future implementations may use out-of-band signaling. Individual vendors will be able to add value to their products by creating applications that make best use of the hub management standard.

Once the standards setting bodies accept the hub management recominendations, manageable hubs will be possible. For example, a manager could prevent access is certain resources or prevent a defective component from affecting the entire network. A network manager could use the hub's ability to enable and disable ports to alter the configuration of the network. Intelligent applications could be developed to take these actions automatically, notifying the operations staff after isolating the fault.

Layer Objects OSI resource objects model the management characteristics of OSI resources such as ISDN resources and FDDI resources. The layer groups in the Regional Workshop Network Management (RWNM) will take the lead in profiling these objects in conjunction with the work to profile the resources that they model. Lower layer (layer 1-6) objects are to be developed by ISO/IEC/JTCI/SC6.

Now that a critical subset of system management standards have reached DIS, the infrastructure for the development of managed objects is stable. There is a clear market need to develop products for network management based on stable ISs. For implementors to develop interoperable management products based on ISO management standards, there 
is an urgent need to produce profile agreements tor layer managed objects. Since the process of developing agreements is likely to take several years, it is important that the work on managed object agreements begin as soon as possible.

The Transport and Network Layer management standards under development by SC 6 have recently reached DIS. These DISs include managed objects that are quite stable. The Lower Layer Special Interest Groups (LLSIG/EG-LL) of the regional workshops should begin development of agreements for Transport and Network as soon as possible to meet the urgent market needs for products based on such agreements. Managed objects for lower layers should complement current lower layer profiles rather than being developed separately.

Real Objects A model has been proposed by OrW NMSIG for defining managed object classes related to computer platforms[79]. The philosophy behind the proposed model is to define a composite or aggregate managed object class called "computerSystem" that provides a high level view of a computer system, including its physical and logical, as well as its hardware and software components. Detailed views of these components are then modelled as object classes contained within the computerSystem object class, as shown in the CONTAINMENT TREE in Figure 34 (N.B.: This is NOT an inheritance tree.). A great benefit provided by this model is flexibility. As and when more computer components need to be specified, they can be defined as individual object classes and "plugged" into the above structure under computerSystem, without upsetting the other object classes. 
(draft)

OIW NMSIG computerSystem

CONTAINMENT TREE

( nmsig-computerSystem )

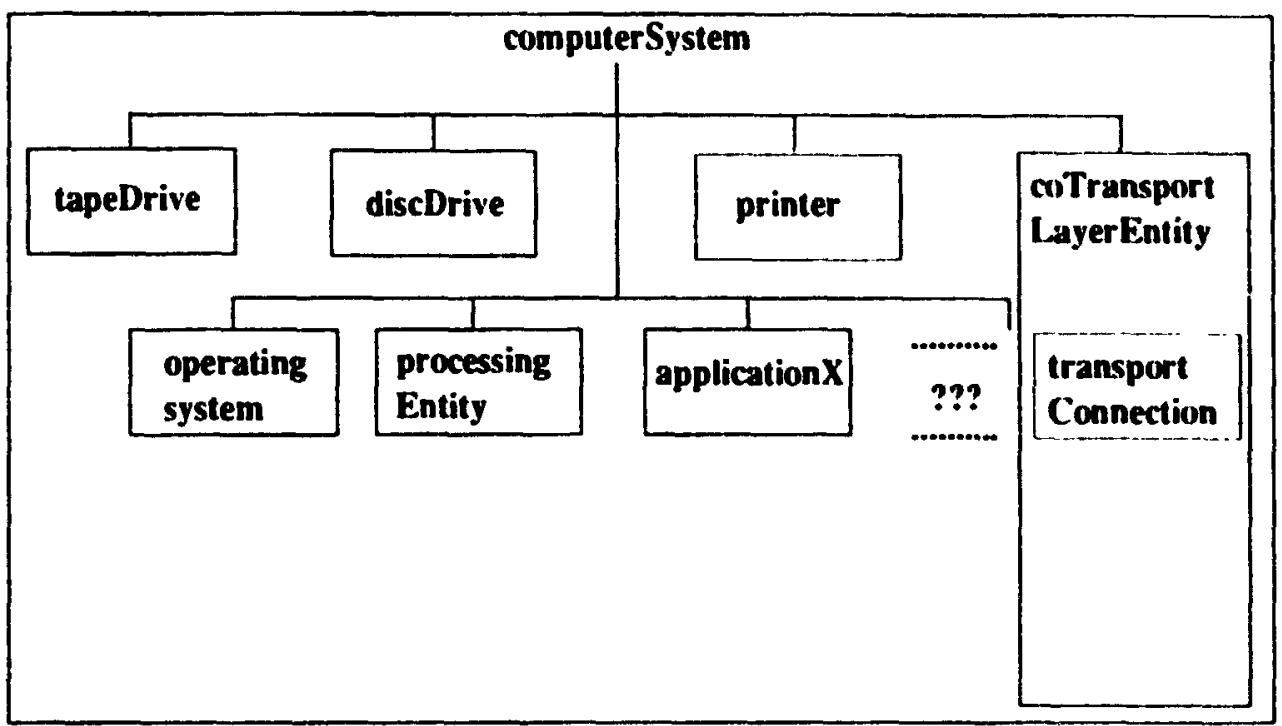

nmsig-computerSystem MANAGED OBJECT CLASS

DERIVED FROM "ISO/IEC 10165 - 2": top ;

CHARACTERIZED BY

nmsig-computerSystem-Package

PACKACE:

REGISTERED AS ( nmsig-objectClass 2);

\section{Figure 34 OIW NMSIG Computer System}

Registration One of the key issues regarding management information is how and where to register and maintain management information definitions. Management information, for classes of network resources, needs to be defined, registered, and ultimately placed into standard libraries so that common views of network resources will be available to all network management users and implementors. A problem is arising in the OSI registration arena, where various organizations are defining and requesting registration 
for different interpretations of the same objects. One of the key points relates to the need to appoint and maintain a recognized intemational registration authority to keep track of the objects being defined and insuring their formal correctness and compatibility before accepting them into a library. This would prevent the unnecessary proliferation of management information structures, minimize the number of redundant managed object classes and promote the use of proven managed object definitions. U'sers and product developers could then search a library and find the definitions of the managed objects that fulfilled their needs.

To date, however, although several are planned, few standard libraries are available. The following are just some of the definitions which have used the GDMO guidelines with no guarantee of uniqueness:

- 1. OSINM Forum 006 Library Supplement:

- DIS GDMO Translation

- Issue 1 Draft 4.0

- January 1991

- This is a complete translation of the Release 1 NM Forum Library from its original NM Forum Object Definition Guidelines (a simpler set of guidelines, developed when GDMO wasn't stable), to the DIS GDMO format. Note that some restrictions from the original Forum guidelines still apply, but the GDMO format is correct.

- 35 Object Classes, 275 Pages

2. ISO/EC JTC1/SC21 N4960 Generic Managed Objects. 
- 5 Object Classes, 15 Pages

- 3. ISO/IEC DIS 10165-2 Definition of Management Information [51]

- Defines commonly used Management Support Objects

- 15 Object Classes, 73 Pages

- see above

- 4. ISO/IEC JTC1/SC21 CD 10164-12 Test Management Function [58]

- 2 Object Classes, 30 Pages

- 5. ISO/IEC CD 10164-11 Workload Monitoring Function [57]

- To manage utilization.

- 2 Object Classes, 44 Pages

- 6. ISO/IEC CD 10164-13 Summarization Function [61]

- 6 Object Classes, 45 Pages

- 7. ISO/IEC CD 10164-9 Objects and Attributes for Access Control Security [60]

- 3 Object Classes, 16 Pages

- 8. ISC/EC JTC1 CD 10164-10 Accounting Meter Function [56]

- 3 Object Class..., 34 Pages 
- 9. NIST NMSIG - 90/173 Management Information Library A variety of MO Classes, some of which are duplicates of the Forum (so far). It is a repository for definitions and will be a good source in the future.

- 14 Object Classes, 50 pages

- see above

- 10. CNMA (Communications Network for Manufacturing Applications) Management Information Library (can get from NIST NMSIG-90/196)

- 24 Classes (some duplication with ISO and the Forum) About 100 pages

- 11. NMSIG Contributions To IEEE 802 (can obtain from NIST NMSIG-90/197)

- defines objects for 802.2 LLC, 802.5 MAC, 802.3, and 802.1 LANBRIDCE:

- 13 Object Classes, about 50 Pages

- see above

- 12. ANSI T1.214 Generic Network Model

- 18 Object Classes, 42 Pages

- 13. IETF OIM MIB (this document can be obtained off the Internet - "sce.carlton.ca" /tmp_mnthome/sirius/wta/ff/RFC1214.TXT.Z 
- It is the Internet MIB II for SNMP, converted to GDMO format for CMOT applications. Generally, these are simpler definitions because there originals did not include events and actions. There does not appear to be any use of Packages either.

- 11 Object Classes, about 120 Pages

- see above

- 14. IEEE 802.3 Hub Management. This document is in the draft stages (as are many of the documents listed above). It defines MOs for Smart Wiring Hubs (Intelligent Multi-port Repeaters).

- 7 Object Classes, about 40 Pages

- see above

Catalogue SC21/WG4 is proposing the establishment of a Managed Object Advisory Group which would review all draft definitions of managed objects and other elements of management information with the objective of identifying possible areas of commonality with definitions completed or underway in other groups. Hopefully, the joint effort of the three Regional Workshop Network Management (RWNM) groups, OIW NMSIG, EWOS EGNM, and AOW NMSIG on an International Management Information Catalogue (IMIC) will aid in this regard. A policy being developed in the NMSIG advocates not defining new objects without first checking if definitions for them already exist or are in the process of being specified. As well, the OSINM Forum is working towards the establishment of the IMIC for OSI based managed objects, to be governed by the Regional Workshop Coordinating Committee (RWCC) and operated by an appropriate 
administration agent. Once the catalogue exists, formal procedures must be established to insure the conformance of developed objects to their definition. Object definitions in the library must also be supplemented by the Object Implementation Conformance Statements (OCISs), which will be the basic reference for the real testing of the objects.

There is also a plan for a jointly developed "Catalogue of Management Information" where one can find directories to examples of such management information. It may be useful to include in this catalogue an indication of the availability of test cases for the managed object. Readers would then have insight into whether or not conformance tests for the managed object are available. Objects for which test cases are available would probably be more desirable. Other potentially useful pieces of information that could be attached to each catalogue entry include date of initial entry into the catalogue and date of last change to the catalogue entry. This information could be used by readers of the catalogue to assess progress in completing the definition and registration of each catalogue entry.

The OSI Directory [41] is expected to support both a white pages service, in which names and their values can be looked up, and a yellow pages service, in which names can be retrieved according to their attributes [11]. The OSI Directory could be used to store names and addresses of managed object instances, though such a mapping between containment hierarchies and The Directory has not yet been defined or standardized [2].

There are issues involving cost and availability of managed object class definitions. Managed object class definitions should be "publicly available" (i.e. easily available, free or very low cost). It is desirable that the catalogue be made accessible in electronic 
form. Another issue is whether there are any copyright or uther legal restrictions from using "private" but publicly available managed objects classes.

Conformance A taxonomy of managed object classes for conformance is being considered by OIW NMSIG. These conformance classes would be specified to delineate conformance requirements for a well-defined and bounded set of management capabilities and features. Once a conformance class is delineated which specifies the set of requirements for that class, tests can be developed to evaluate conformance of products to that conformance class. The development of conformance classes will enable users to define procurement specifications, vendors to define management capabilities and features, and conformance test houses and others to define test cases. This is a critical issue as the standards do not define object behaviour in a formal way and it is difficult to precisely state what testing an object's conformance really means. This is of prime importance for the area of fault management, confidence and diagnostic testing.

\section{Interoperability testing}

Well advertised and readily available access to ready-to-use, conformance te ited network management object definitions will do much to halt the roll-your-on approach that is prevalent at this point in time within the OSI network management development arena. The situation needs to be viewed in terms of maximizing interoperability and maintaining the integrity of the object definitions. The whole purpose of OSI is to maximize interoperability and the focus on that goal is sometimes easily lost. The basic idea is to define a package for each SMF that would specify the minimum necessary to 
guarantee interoperability and a base functionality. Objects which claim conformance would have to demonstrate support of these packages. Obviously vendors could extend beyond these packages with proprietary features but all conformant objects would support this common subset. In the shor term, interoperability testing should be considered since it does not appear feasible to develop test cases for all managed object classes (e.g. ISO objects, NM Forum objects, NMSIG MIl objects). Interoperability testing would not require formal test cases and would presumably give assurance that the implementations would interoperate in the real world as well as in the testing laboratory. 


\section{CHAPTER 6 Research Summary and Future Research}

The problem that this :hesis has addressed is the technology gap that exists between user expectations and the communications networks' inability to live up to their promise because of the networks' unmanageability. The solution that has been presented is the evolving OSI network management standards from the perspective of the fault management function.

\subsection{Research Summary}

But, concepts are much more important than methodologies. Only after a concept is precisely defined and understood is it possible to discuss the methodology of its use. Following is a summary of the research, discussed in relation to each of the six objectives of this thesis as outlined in Chapter 1 (1.4):

- To provide a perspective on a relatively new area in which there is voluminous literature

The volume of literature in the area of network management is evident by the large (100 entries) Bibliography cited by this thesis. Each of these references, except as noted, has been acquired for the PEGASUS project by the author during the research for this thesis. The recent publishing dates of the OSI and DARPA Standards illustrate how relatively new this area is. This was challenging as both the OSI and DARPA Network Management Standards were moving targets during the period of this research. 
The major concepts are that network management is not well defined as of yet and is evolving rapidly on a very wide front because of significant user demand.

- To collect, synthesize and present the current OSI and DARPA network management standards

By reading and absorbing the collected literature, a synthesis of latest available OSI Network Management Standards was presented in Chapter 2 of this thesis. The context was established by presenting the history of tive ISO and its standardization process. Then the relationships between the OSI documents were illustrated in Figures $3 \& 4$, and $7 \&$ 8. Next the OSI Network Management Architecture, and Services (CMIS) and Protocols (CMIP) were introduced. The concept of System Management Functions (SMFs) was presented along with details of 8 of the nost important for the Fault Management System Management Functional Area (SMFA). Finally, the Structure of Management Information (SMI) documents were presented, emphasizing the application of object-oriented design to the formation of a Management Information Base (MIB). The use of Abstract Syntax Notation One (ASN.1) (and its Basic Encoding Rules (BER)) as the standard specification language and the use of OSI Guidelines for the Definition of Managed Objects "templates" (GDMO [ISO 10165-4] [52]) as a set of standard templates for defining managed object classes (MOC) are the major concepts of Chapter 2.

By reading and absorbing the collected literature, a synthesis of latest available DARPA Network Management Standards was presented in Chapter 3 of this thesis. The cuntext was established by presenting the history of the DARPA and its standardization process. Then the backgiound and root documents of the DARPA Network Management 
Standards were summarized. Next the DARPA Network Management Services and Protocol (SNMP), and Architecture were introduced. Finally, the Structure of Management Information (SMI) documents were presented, emphasizing the concept of simplicity t1 the formation of a Management Information Base (MIB). The use of a subset of Abstract Syntax Notation One (ASN.1) (and a subset of its Basic Encoding Rules (BER)) as the standard specification language and the use of a standard format (OBJECT-TYPE macrus [RFC 1155] [73]) for defining managed objects are the major concepts of Chapter 3.

- To serve as a tutorial on and reference to the latest available OSI and DARPA network management standards documents and terminology

The presentation of the synthesis of the collected OSI and DARPA literature of Chapters 2 and 3 resulted in a tutorial on both of the network management approaches. The tutorial was reinforced by the comparison of the two approaches that was given in Chapter 4. Chapter 5 (5.3.4) illustrated the application of the OSI SMI to the IEEE 8(12 LAN Bridge. The concepts of containment, registration and inheritance were covered in detail. The tutorial was supporter by the reference to the latest available OSI and DARPA documents that are contained in the Bibliography. The relationships between the OSI documents were illustrated in Figures $3 \& 4$, and Figures $7 \&$ \&. A Glossary of over 150) terms from both domains augments the tutorial and reference value of this thesis. An Index of the terms is included under the major topics: General; DARPA; and ISO.

- To compare the OSI and DARPA network management approaches 
Chapter 4 began by comparing the OSI and DARPA protocol stacks in relation to a generic 7-layer OSI Basic Reference Model. The essence of the comparison was based on arguing the costbenefit trade-offs of having more complex or simpler underlying primitives. This difference was more concisely stated as the "complete aspects" of OSI (CMIP) versus the "core aspects" of DARPA (SNMP).

The comparison was based on data access philosophy, the efficiency of OSI reporting versus DARPA polling, the functionality of CMIP versus SNMP, and size and performance. The basic concept was that OSI network management is an application that is serviced by a connection-oriented, 7-layer protocol stack, while DARPA network management is an application that is serviced by a connectionless, 4-layer protocol stack.

It was argued that from the network operator's perspective, the protocols are equivalent as the same network management application can be built upon either one. In fact, equivalent functionality could be provided using both protocols if a mixed set of management agents were to be employed.

But as the global network increases from hundreds to thousands and tens of thousands of devices, the bandwidth needed for SNMP activity will degrade WAN service to user traffic. The scalability of SNMP is in question - now! The initial cost in overhead and the current unavailability of implementations put CMIP in question -now!. Users want network management capabilities, but not if it substantively increases price or decreases performance. This a the main argument of the developers of SNMP. SNMP's simplicity gives better cost/performance-now! How long will that last ?

However, this author believes that more important than protocol is the issue of the 
representation and communication of network management information between network managers, agents, proxies and network elements. Chapter 4 continued hy comparing the OSI and DARPA Structures of Management Information (SMIs). Again, this difierence was more concisely staied as the "complete aspects" of OSI (GDMO "templates") versus the "core aspects" of DARPA (OBJECT-TYPE macros). The comparisun uas based on representation (OSI uses ASN.1 and its BER, while DARPA uses a subset), mudeling (OSI is object-oriented, while DARPA is not), security and coexistence. Figure 17 illustrated this comparison in tabular form.

This thesis stated that the introduction of both OSI ASN.1 and its BER II DARPA is a fundamental concept, and together they should allow future applications to be portable between the two environments. The author believes that the IEEE goal of protcriol independent managed object definitions, as discussed in Chapter 5 (5.3.4), should be standardized in order to minimize the development and conversion efforts needed in the future of interoperable network management.

The author has concluded that the correct approach for object specification is to define and register new MIB objects with GDMO (OSI) and let the Internet (DARPA) versions (subsets) be derived from them. This would ensure that all MIR definitions - - which really just define manageable resources, without dependence on management protucouls - were aligned across whatever management protocol or management system was used by an administrator for managing an environment.

The final conclusion was that, in reality, both protocols will occupy certain segments of the market, and that a solution to accommodate both s needed to achieve true 
integrated end-to-end network management. The important requirement from the interoperability point of view is that the in:trumentation in each agent have the same semantic:, irrespective of which network management framework is used to access it.

- To relate an example real world network fault management environment to the non-communications specialists of the TRIO sponsored PEGASUS project

Chapter 5 began with an overview of network fault management, its requirements and functions. T: $n$ a context for fault management was presented in Section 5.2 with an example real, third generation network environment. Next, in Section 5.2, the major network elements (hubs, routers, gateways, etc.) were described in relation to the sample network and to the OSI Basic Reference Model. Then the example real fault management implementation was described in Section 5.3. PEGASUS and some previous fault management approaches were introduced. Finally, an application of the OSI SMI and GDMO to an example network element (IEEE 802 LAN Bridge) was presented in 5.3.4. A Glossary of over 150 terms from the telecommunications and network management domains was included to aid non-communications specialists of the TRIO sponsored PEGASUS project.

- To present a possible migration path for the example network from DARPA network management towards OSI network management .JARPA->OSI)

Chapter 5 (5.3.3) introduced Version I of the U.S. Govemment Network Management Protile (GNMP) which requires CMIS/P and 5 general OSI system management support functions (SMFs). Figure 24 illustrated how the author envisioned that GNMP could 
be applied as an Interim Network Management solution that would permit a phased migration from the example DARPA-hased network to an OSI-based network. Figure 2.3 distinguished areas of standardization from those areas unlikely to become standardized in the near fiture. These were seen to be within in the domains of XNETMON and PEGASUS, and represent areas where future research is needed. This author concludes that there is nothing to preclude the application of PEGASUS to an OSI network or the OSI network management standardized approach.

\subsection{Future Research}

This section of the thesis suggests areas of research in the non-standardized domains of the Interim Network Management (INM) System of Figure 24. These areas are the Human-Machine Interface (HMI), Data Base Management Systems (DBMS) and the Analysis Functions as illustrated in Figure 23.

\subsubsection{Object-Oriented Human-Machine Interface (HMI)}

The object-oriented paradigm is proving successful in the presentation area of the Human-Machine Interface (HMI). In the example network of this thesis, XNETMON has an in-house, $\mathrm{C}++$ implementation of an HMI. XNETMON has icons representing the major network elements such as routers, serial links, LANs, HP bridges, Fastpath gateways, fileservers, etc. The network topology can be drawn on the network monitor display, and the statuses of network elements are represented by colour conding the icons.

In order to manage more complex networls, communication network operations will integrate more functions in the future. Exiending the capability of XNETMON to include 
the diagnosis of the components of these network elements will require more detailed images and the visualization of more complex relationships. As well, the HMI will be required to initiate, display and evaluate network element component testing during the diagnosis process.

Being able to decrease human errors during diagnosis will be an important factor. HMIs are essentially regarded as the link between a user cognitive model and a system designer's conceptual model of a systems's functions, capabilities and limitations [85]. The errors at the intention-forming stage in the problem solving model are the most serious errors. At the intention-forming stage, one of the most important factors is the method of displaying the outcome [101].

In the abstraction, modelling and implementation of image representations for fault management systems, it is important to maintain consistency with the concepts defined by the OSI Standards discussed in this thesis. The object-oriented model enables the use of a common definition as defined in the GDMO [52]. Uniformity of representation means that every concept is directly represented (visualized) using the same basic techniques. The user and the computer will dynamically manage the components in a symbiosis which has the effect of amplifying the native intelligence of the user.

From the HMI point of view, the problem is to identify a set of logical visual objects representing how the physical network elements are seen from the network operator's perspective. This is a similar mapping to the logical from the physical as addressed by the OSI SMI ( [50], [51] and [52] ). Adopting the inheritance principle of the ubject-oriented model, these managed object definitions might be reusable from the HMI 
viewpoint. This would create a continuous thread from the physical object through the MIB to the HMI into the conscinusness of the human operator. Relevant HMI oriented properties (e.g. adding a colour attribute to alarm thresholds) can extend the GDMO managed object definitions. It is proposed that the adaptation of OSI SMI developed managed objects to the HMI could be a useful area of network management research.

\subsubsection{Data Base Management Systems (DBMS)}

Just as the OSI Standards do not define the "look and feel" of the HMI, the OSI SMI does not describe how to implement a MIB within a database (see Figure 23). In the example network of this thesis, XNETMON incorporates an in-house developed database accessible by the $\mathrm{C}++$ application code. The database is dynamically contained in memory during XNETMON execution. At the initiation/termination of XNETMON, this database is read/written to/from a persistent flat-file on disk storage, with traditional data processing tape medium for backup and restore. The network administrators prefer that XNETMON be converted to a commercial"y available database management system (DBMS). The goal is to allow more flexible report generation and multi-user concurrency to a single XNETMON database with as little impact on the $\mathrm{C}++$ application coude as possible. High data capture rates and data integrity are mandatury. Flexible and easy extraction of information, including topological and configuration data, is a valuable asset. The problem is one of implementing the current object-oriented $\mathrm{C}++$ models (and of the SMI and GDMO, in the future) into an efficient and reliable persistent store of this information. 
Currently, there are two models of DBMSs that could be cho" support XNETMON: a relational database management system (RDBMS) or .n: t-oriented database management system (OODBMS). Both approaches have ac'vantages and disadvantages. Regardless of the model chosen, fundamental database functionalivy such as large persistent data capacity, concurrent access, language support, as well as security aspects of access, backup and recovery are required. Other considerations are the hardware/software platform and the central/distributed placement of the database(s).

In the relational model, relationships are set up by embedding identical values into related entities. With object-oriented approach, entities can be related independently. However the advantages of relational databases are many. Relational technology is mature, stable and is supported by many suppliers for many platforms, including SunOs/Sparc. Relational products support large persistent data capacity, concurrent access, as well as security aspects of access, backup and recovery. Referential integrity is a common feature of relational database management systems. These mechanisms assure that references betw'een objects (e.g. semantically understood to be a containment relationship) are consistently maintained. Structured Query Language (SQL) is a standard access language for relational databases. This permits easy prototyping and implementation of application activities such as report generation and ad-hoc querying. Performance tuning of relational databases is well understood.

A facility to create and manag: user-defined types is one of the important features typically missing from most relational database management systems (RDBMSs). As well. object-oriented models do not map well. Both inheritance and containment rela- 
tionships must be "flattened" from their tree structures into tabular relations for storage within a relational database. In Chapter 10 of [5], Booch develops the concept of capturing a relational database schema in $\mathrm{C}++$. Class diagrams are designed that map directly to entity-relationship (E-R) [16] diagrams. The relational database supports embedded functions "called" from the $\mathrm{C}++$ program. This example is a centralized database accessible through a distributed network as in the clienu/server model. For example, Figure 35 illustrates the class inheritance structure for alarm reporting that it is proposed could be implemented using $\mathrm{C}++$ with embedded SQL statements for a commercially available relational database management system. 
CLASS IVHERITAVCE

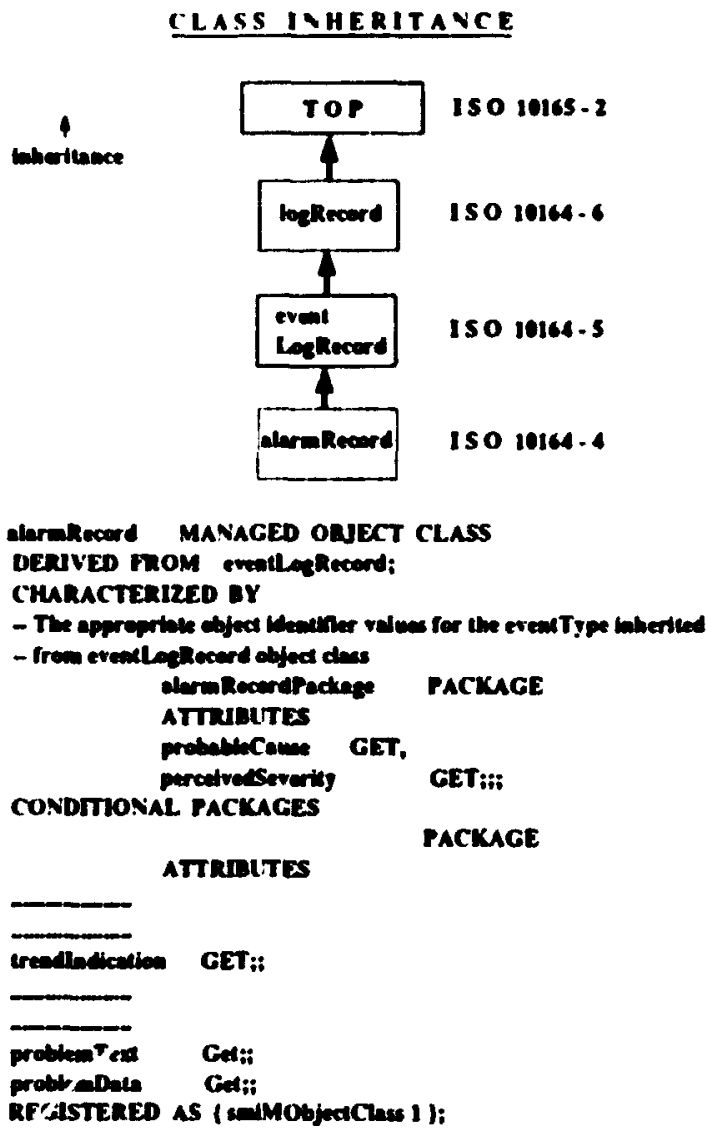

Figure 35 Alarm Record Class Inheritance

There is a gap between the dependable hardware and software that drives corporations (e.g. RDBMS) and the tempting technologies of the future (e.g. OODBMS). "Although object-oriented database technology is expected to achieve stability soon, it may not be mature enough at this point to be considered in a product implementation," [2]. Most corporate data still reside in RDBMSs. Because network management and the mission of the XNETMON application are critical to this corporation, it is proposed that 
a commercial relational DBMS be adapted to the ubject-oriented aspect using $\mathrm{C}++$ as an "impedance matcher".

\subsubsection{Analysis Functions}

But, even with the best methods of collecting data from a network, a network management system is of little use if that data cannot be used for analysis. In the example network of this thesis, XNETMON does not perform any online analysis function. but acts as an operator focused display of the network's status. The XNETMON ling is available for offline report generation of network status.

The first step in any analysis (diagnostic) process consists of accurate recognition of the phenomena confronting the network manager. The second step is proper labeling of these phenomena so that an accurate, succinct portrayal of features of the presenting problem can be made. It is vital that a common standard technical language be used when communicating about network problems. For network management, the terms and data structures in the OSI SMI and the SMFs are the vocabulary of this standard technical language. These terms aid in discriminating one phenomenon from ano:her: sometimes correct diagnosis will hinge on the proper distinction of these terms. The third step is systematic ruling in or out of alternate faults, which might produce similar ubservations.

To aid in this second step of labelling the distinct features of a network problem. a multiaxial, statistical classification scheme is proposed. A statistical classification indicates the relationship between diagnostic categories and must be confined to a limited number of categories that encompass the entire range of the problem domain. The basic rule with a multiaxial system is that each axis is considered for each individual case and 
the findings, or absence of findings, ars so documented. The model for this multiaxial, statistical classification is taken from the Diagnostic and Statistical Manual of Mental Disorcurs (Third Edition), DSM-III [92]. To make it workable, the system is restricted II a small number of axes that were chosen on the basis of providing information of maximum usefulness in the greatest number of cases.

ISO IOIM-A ALARM REPORTNG PARAMETERS

\begin{tabular}{|c|c|}
\hline Axs & Poreneter nave \\
\hline & 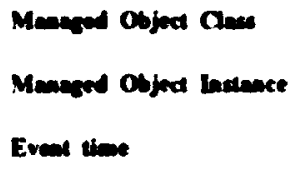 \\
\hline 1 & Alem type \\
\hline $\mathbf{n}$ & Probable canae \\
\hline $\mathbf{m}$ & Pareatived enverits \\
\hline IV & Trued indicatien \\
\hline $\mathbf{v}$ & 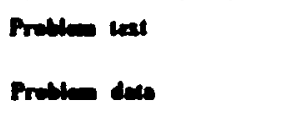 \\
\hline
\end{tabular}

Figure 36 Multiaxial Proposal

If the alarm recurd from Figure 35 is analyzed for content, it exhibits a multiaxial structure. Figure 36 illustrates a proposed division by axis of the alarm report parameters as defined by ARF [46]:

- The individual managed object causing the alarm is identified by its managed object class and its managed object instance (DN or RDN). 
- The time of the alarm (event time) is defined in generalized time type (GeneralizedTime), with the precision of the time representation indicating the granularity of the time measurement (i.e. YYYYMMDDHHMMSS.SSS and an indication of the time differential (HHMM) between local and universal time can be appended).

- Axis I-alarm type with values [0..4]

- Axis II- probable cause with values [0..44]

- Axis II-perceived severity with values [0..5].

- Axis IV-trend indication with values [0..2]

- Axis V-a combination of human readable problem text and problem data.

What is cicar is the analysis of alarm records both online and offline as specialized logged records can be enhanced by applying the multiaxial approach. The concatenation of Axes I. II, II and IV yields a five-digit code. No diagnosis would be complete without enough information to provide for five digits. With a multiaxial approach, diagnosis is based on specific criteria for each alarm so that when a given diagnosis is used, it can be known quite exactly what is meant because the precise diagnostic criteria that have created the alarm are known. There is still room for experiential judgement using Axis V. The automated analysis of alarm report data using multiaxial, statistical analysis is proposed for future research in fault diagnosis.

\subsubsection{OSI Network Management Development Environment}

Both PEGASUS and XNETMON, discussed in this thesis, operate in DARPA TCP/P environments. Future research in network fault management will require an OSI and CMIS/P environment. It is strongly recommended that the "Fault Manage- 

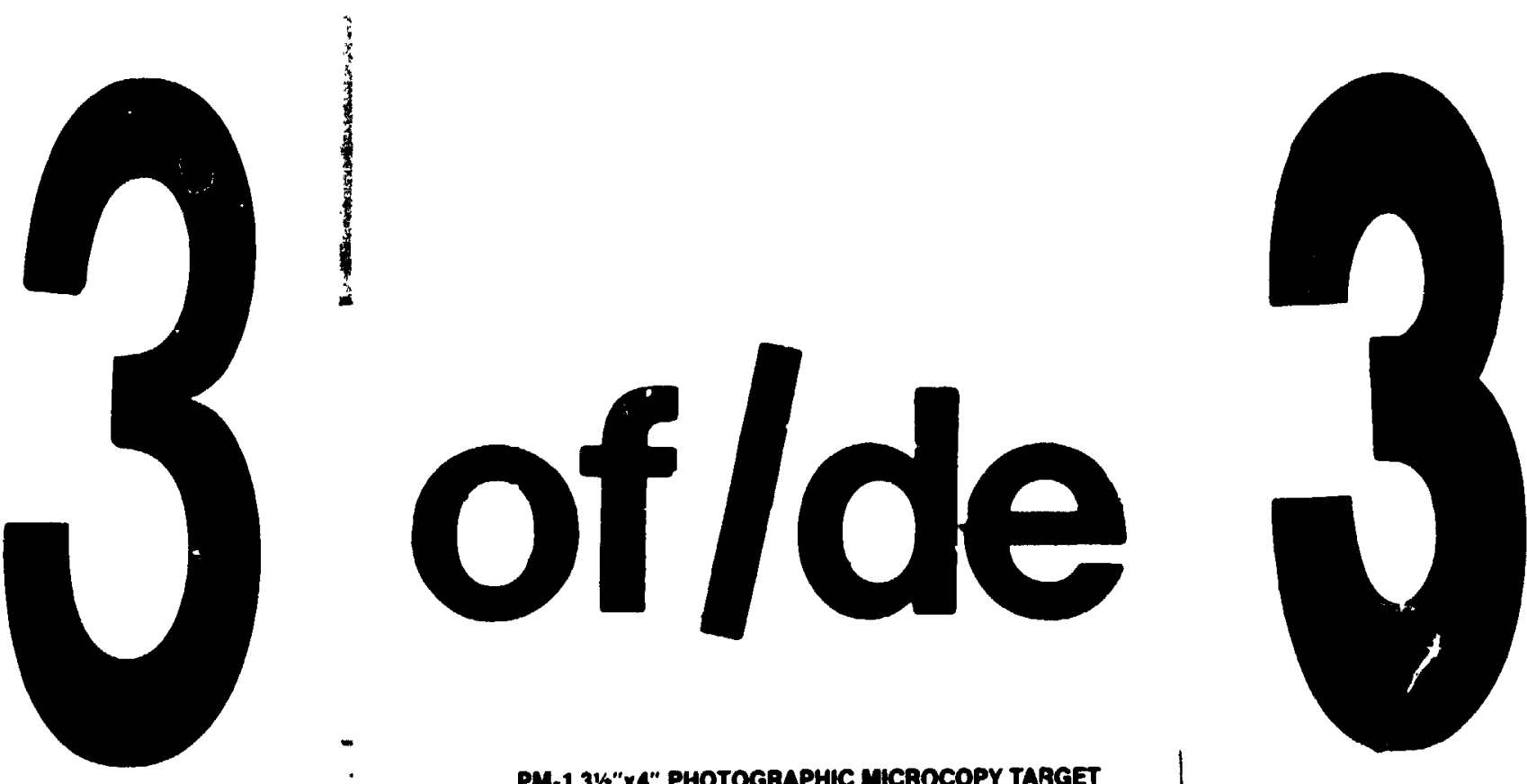

PM-1 31\%" $\times 4 "$ PHOTOGRAPHIC MICAOCOPY TARGET NES 1010 ANSI/ISO "2 EOUIVALENT

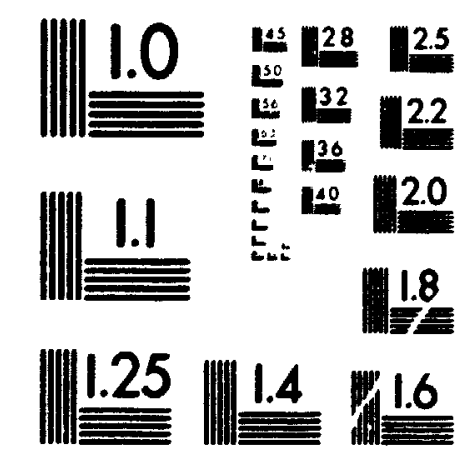

PRECISIONSW RESOLUTION TARGETS

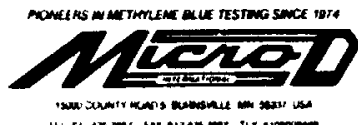


ment in Communications Networks" project contact University College London (osimisrequest@cs.ucl.ac.uk) (see Chapter 6 (What's Happening in Europe ?)) and implement the latest OSIMIS (OSI Management Information Service) packije version 2.95 from UCL [64].

OSMMIS is an OSI-based management system developed at University College London. It does not intend to provide a comprehensive set of OSI management facilities but to show how the rich OSI Management functionality can be exploited and to provide a generic OSI management platform. The management service/protocol CMIS/P, based on the International Standard version 2 of 1991, have been implemented using the C programming language while the applications have been developed in $\mathrm{C}++$ in order to exploit the object oriented nature of the OSI Network Management model. A X-Windows based MIB browser has been implemented using the Inter:Views $\mathrm{C}++$ library version 2.6 , which is the only object library used. The example MIB implemented comprises at present managed objects related to the ISO Transport Protocol and there is management instrumentation to manage the ISODE implementation of the latter. The system has been developed using the ISO Development environment (ISODE) version 7.0.

The ISO Development Environment (ISODE) is a quasi-OSI software application within the public domain. ISODE is a non-proprietary software implementation of some of the ISO/IEC protocols and can support several different types of network services below the transport service (layer 4 ), including TCP. This implementation permits the development of application (layer 7) OSI protocols, such as network management, in a robust and mature internet environment. This allows the luxury of not having to recode 
anything when moving to a network where the OSI Transport Protocol is used. This software also operates over pure OSI lower layers as well. ISODE is written entirely in the $\mathrm{C}$ programming language and runs SunOs release 4 as well as several other operating systems. ISODE needs a K\&R or ANSI C compiler. There is no support for $\mathrm{C}++$. The discussion group isode@nic.ddn.mil is used as an open forum on ISODE. Registration on this list can be obtained by contacting isode-request@nic.ddn.mil .

UCL have proposed that implementors and users of OSI management software exchange information on their experiences and share software based on a common platform. For example, it would be very useful tc trade implementations of specific managed object classes. In order to promote discussion of these issues, UCL have set up an intemet mailing list called osimis@cs.ucl.ac.uk. It is recommended that the PEGASUS project subscribe to this list by sending a request to osimis-request@cs.ucl.ac.uk.

\subsubsection{The Future of Network Management in the 90s}

Standards-Enabling or Controlling? The question has been asked as whether the standards-driven approach is beneficial or not. It is true that standards can be, have been and are being used politically by governments to protect their industries and (perceived) national interests. Often company representation on a standardization working group is motivated by the desire to manipulate the process for market advantage or to sabotage the process when deemed beneficial.

At the same time, however, the industry-driven apjroach is not without its politics. Proprietary solutions and customer-control have been the strategy of the information 
technolczy producers since the beginning of the industry.

Whichever the abuse, it is the consumers of the products who suffer most. Finally, during the last few vears, there has been a rebellion in the market-place. The international consumer market is demanding standardization of products. Consumers are voting for standards with the mightiest voice they possess-their money. This author believes that the message is getting through. (Witness the fall of IBM from its Olympus and the mass industrial conversion to the "open system" religion.) The market-driven approach now predominates.

The question then becomes how do the interested parties (government, industry, customers and standards bodies) focus on those activities that address the common parochial problems. Recall from Chapter $2(2.1)$ that the normative standards tell "what" should be standardized, but not "how" they should be standardized; and that the functional standards define the combinations required to achieve a specific function-the "how".

Standardization Will Work ! Citing a need for cooperation among the various groups involved in network management, the OSINM Forum announced in April 1991 that work had begun on producing a comprehensive industry road map. The road map will consolidate all of the different specifications, base standards, profiles and agreements into a single cohesive plan.

Because standards development is an evolutionary process, it represents a moving target (as this author has discovered). Implementation specifications, such as the F(rum's Release 1, "intercept" the standards at a particular point in time and provide stability for 
product development and procurement. Eventually, as a standard matures, it becomes necessary to migrate any interim solution to a new intercept point. The ideal is for all of the various solutions, including Forum specifications, government procurement guidelines and other consortia's work to arrive at a common intercept (OMNIPOINT) which has been agreed upon in advance. It is these intercept points that the road map seeks to identify.

The intent of the road map is to synchronize the industry globally and provide it with a common vision in order to prevent geographical and industry divergence. If successful, the road map will create a stable, confident environment, beginning with OMNIPOINT1, in which suppliers can develop OSI products and users can procure them. Getting products into the hands of users in a timely manner has been the major problem with OSI since its creation and the mission of the Forum since its inception.

The Forum has spoken with other groups and consortia involved in network and systems management about participating in the mapping process. Combined with input from the Forum's User Advisory Council, expect this to be an all-encompassing document. By working together, the parties agree that convergence points between standards and products can be defined.

This road map should be viewed as a blueprint for industry to follow. To be successful, though, it requires the cooperation and participation of all the various groups and factions, including governments, manufacturers, carriers and network administrators. It must take into account all of the separate efforts that exist today and harmonize them into a workable strategy everyone can support and follow. In this author's opinion, it 
is this spirit of international common good and consensus with which stundardization should be viewed and given the benefit of the doubt.

What's Happening in the United States (U.S.) ? While most U.S. users agree that OSI will eventually predominate as a networking architecture, few have concrete plans to adopt it and even fewer have actually implemented it. The overall feeling in the U.S. has been that OSI is not complete and that few OSI products are available. The pace of adoption of OSI in the U.S. is relatively slow.

However, in August 1990, the U.S. government began requiring OSI compliance for all its computer contracts; this strategy is known as GOSIP. The May 1991 version (Version 1) of GNMP (as discussed in Chapter 5 (5.3.3)) specifies an initial, useful set of standards for standard, interoperable network management products capable of operating over OSI networks. These requirements are likely to speed up the offering of OSI products by major vendors, for whom government contracts represent a substantial part of their revenues. The Computer Aided Logistics Support (CALS) initiative becomes operative for the U.S. Defense industry during 1992. Second versions of U.S. GOSIP and GNMP will add function then. So, 1991 has seen some upswing, with 1992 promising to be a banner year for OSI products.

What's Happening in Europe? In general, OSI has been received more readily and enthusiastically in Europe, where the multinational character increases the need for standards. European networking vendors are further ahead with products that meet this 
increased demand. While U.S. users tend to view OSI as costly, European users see immediate cost savings by adopting OSI.

In general, the European OSI market is as much as five years ahead of the U.S. market. In Europe, the pace is accelerating, spurred by the 1992 date for the European Economic Community (EEC). For instance, the previously recommended OSIMIS (see Chapter 6 (6.2.4)) has been developed as part of University College London's participation in the following projects funded by the EEC:

- the ESPRIT I project INCA (Integrated Network Communications Architecture);

- the ESPRIT II project PROOF (Primary Rate OSI Office Facilities); and

- the RACE I project NEMESYS (Network Management using Expert Systems).

OSIMIS is being used in:

- the PROOF project to manage an IP (Internet Protocol) to Primary rate ISDN gateway;

- the NEMESYS project as part of a prototype for Traffic and Quality of Service Management in $\mathrm{IBCN}$; and

- the local UCL environment to monitor ISODE applications' activity.

UCL knows of two OSI management systems exhibited at Telecommunications '91 in Geneva that are based largely on OSMIS.

In Europe the implementation of OSI has also accelerated due to the unification of Germany and the recent collapse of the U.S.S.R. Most East block countries must replace their technology completely. As more European corporations adopt OSI, their subsequent 
savings and competitive edge may well push the adoption of OSI in the U.S. to keep pace with the more standards oriented European marketplace.

What's Happening Globally ? The OSI/NM Forum have aiready held preliminary discussions with the OSI Implementors Workshop (OIW NMSIG), Asia-Oceania Workshop (AOW NMSIG) and European Workshop for Open Systems (EWOS EGNM). These Regional Workshops play a pivotal role in converting base standards into implementation agreements, and much of their work forms the basis for government procurement pro. grams (GOSIPs). The three Regional Workshops groups have resolved to have joint meetings and to identify them as RW-NM-CC (Regional Workshops Network Management Coordinating Committee) meetings, following the example provided by RWCC meetings.

RW-NM-CC meetings are aiming at resolving technical issues in the development of joint work such as taxonomy and profiles for Network Management. It is believed that joint meetings will provide the adequate structure for progressing technical work and ensuring coordination while making the best use of available resources. RW-NM-CC meetings will be held when enough preliminary work has taken place in the workshops, and issues have been identified, so that significant results can be expected from a joint meeting. An estimation for the short term is that these meetings will be held every 6 to 9 months. They will be hosted by every workshop in tum. The objective of RW-NM-CC is to facilitate the technical development of joint texts for Network Management taxonomy and profiles and any other material requiring international harmonization in the area of network management. 
The RW-NM-CC is currently examining the availability of expected base standards to plan for the development of corresponding profiles. June 1992 is the planned date for the first harmonized recommendation documents to be submitted to ISO. Therefore, initial certified network management products conforming to IS level ISO Network Management Standards can be expected before the end of 1992.

Criteria for a Network Management Choice The following in the author's considered opinion are the major criteria for choosing either OSUCMIP or DARPASNMP as one's preferred Network Management standard. Each criterion points towards a particular standard but it is the combined effect that is required for the decision.

- Al-If there is a single network domain, the NM choice can be either CMIP or SNMP. The decision should depend on the network technology chosen, i.e. DARPA or OSI. - A2-The more domains that are internetworked, the orientation should be towards OSUCMIP. OSICMIP has more functionality to better manage multiple domains.

- B1-If the networks are homogeneous the decision should depend on the common network technology.

- B2-The more the networks are heterogeneous, the more the orientation should be towards OSICMIP. OSVCMIP was designed to manage heterogeneous networks.

- Cl-The more the network is Nonth American contained, the more the orientation should be towards DARPASNMP. DARPA is the predominant network technology in North America, except for IBM's SNA. 
- C2-The mure global and international the network, the more the orientation should be towards OSICMIP. OSI is the predominate network technology in non-North American countries and is increasingly so.

- D1-If the network is within a Government or must interwork with a Government network then the orientation should be towards OSICMIP. Most countries have policies (GOSIP, COSAC, etc.) which require OSI network technology.

- El-The further ahead in time the decision, the more the orientation lowards OSV/CMIP. OSI is recognized as the long range network technology. DARPA has a strategic plan to migrate to OSI itself.

The author would describe the sample network in this thesis as:

- $\mathrm{Al} \wedge \mathrm{B} 1 \wedge \mathrm{Cl} \wedge-\mathrm{Dl}{ }^{\wedge} \mathrm{El}(\Delta \mathrm{time}=0)$.

Therefore the network management decision for the sample network should be DARPASNMP for now and for the foreseeable future.

The author's recommendations for the future strategy for this network would be:

- to monitor the rate of announcements of OSI and CMIP products;

- to monitor any decision by the DARPA Internet Advisory Board to begin planning for implementation of the DARPA to OSI migration strategy; and

- to time this future conversion decision on the return of investment in:

- the computer platform hardware, operating system software, applica:ion software and user training; 
- the network hardware, network operating system, network management software and user training; and

- the price/performance of the above due to technological innovations.

As the author's proposed DARPA/SNMP to OSICMIP phased migration strategy shows (Chapter 5 (5.3.3)), OSI will not cause users to replace all their current hardware with OSI-specific products without return-on-investment considerations. OSI will integrate hardware that exists rather than create a new hardware market per se. The compelling strategy for any network manager should be to time this future conversion decision on: the return of investment in the computer platform hardware, operating system software, application software and user training; the network hardware, network operating system, network management software and user training; and the price/per ormance of the above due to technological innovations. It is reality that both SNMP and CMIP will occupy certain segments of the market for some time. In the interim a way is needed to accommodate both in order to achieve tue integrated end-to-end network management.

What Protocols Can Not Do While OSI's potential impact is profound, there is a tendency to exaggerate its capabilities and attribute functions to it for which it was not designed. For example, OSI does not provide an application program interface; rather it provides protocols for applications to communicate information. Additionally, OSI does not specify a user interface such as the Graphical User Interface (GUI). OSI does not specify a standard operating system platform such as is being addressed by the IEEE POSIX standard and being considered by ISO (ISO 9945-x). 
These overall information technology standards are the domain of the Open Distributed Processing (ODP) work being developed by ISO/AEC JTC1/SC21/WG7, of which the OSI Basic Reference Model is only a minor component. The network management problems and the evolving OSI Network Management Standards solution addressed in this thesis are a look into the future of world-wide information technology. Enterprise network managers will need the ability to actively control distribured hardware com. ponents and software modules of all sorts. As well, the design and implementation of management information bases is a specialized subset of the not yet well understound domain of distributed databases.

One part of this thesis has addressed the issue of the choice of the protocol standard to be used to perform network management. The OSI Basic Reference Model concentrates on peer-to-peer communication rather than the description of an arbitrary set of objects to be communicated. The "how" of transmitting information, important as it is, is overshadowed by the issue of "what" standardized information to transmit and how it can be displayed and stored efficiently for analysis and use. The domain of protocol specification, testing, and verification has been well established for over ten years [4]. The development of protocols is relatively under control.

The direct application of the modeling, structure and representation of management information standards, as described in this thesis, is only slowly beginning. The definition of application (layer 7 and above) content is not under control. "The lack of standard management information for the plethora of new manageable resources will loom even greater as the most significant near term impediment to integrated manage- 
ment," [76]. This author believes that the lack of a rich, registered library of publicly available conforming management information objects is a reminder of what remains to be accomplished. There is an immediate need for research in the complex and nascent domain of defining managed objects.

It will be some time before networks will be capable of managing themselves. However, this process will be greatly simplified if the underlying models used to describe these global systems are the same. There should be an increasingly fertile exchange of ideas and requirements within the standards community, between those concerned with an architectural framework for distributed systems and those with particular interest in their management.

A network management station is simply a viewport into a maze of interconnected networks. If the network manager cannot gain insight into every object needed to be seen throughout the network, the maze of the problem domain will never be navigated. This is the final criterion to be applied to any network management system, regardless of the technology from which it is constructed.

Conclusion But what matters now is not the nature of telecommunications, computers and networks, but the nature of ideas. The sustaining and nurturing of the earth and human civilization require basic changes in our socio-economic systems. New technologies that enhance the ability to create and understand information have always led to dramatic changes in civilization. Information technologies are relatively clean and benign. They can be applied imaginatively to both proactive environmental monitoring and reactive disaster control. It is difficult to create innovative ideas, generate knowledge and share 
information.......and it always will be. But at least in the future, global networks and integrated network management will make it easier...... and at the same time free up collective human intellect to create the ideas needed for our real world problem-survival. 


\section{Appendix A Glossary}

Abnormal (test) termination: [58] a statement made with respect to a test invocation when the test is prematurely terminated.

Abstract data type: [50] defines a set (in the mathematical sense) of possible values that constitute the domain of the datatype, and a set of valid operations (in a languagetheoretic sense) that can be performed on these values.

Abstract syntax: a description of a data structure that is independent of machineoriented structures and restrictions.

Abstract Syntax Notation One (ASN.1): the OSI language for describing abstract syntax which has developed as a lingua franca for the representation of arbitrary data structures and is defined in ISO 8824 [35].

ACSE: Association Control Service Element.

\section{AE: Application Entity.}

Agent: [42] a MIS-1ser (application) which for a particular exchange of systems management information has tiken an agent role.

Agent role: [42] a MIS-user (application) taking an agent role is capable of performing management operations on managed objects and of emitting notifications on behalf of managed objects.

Alarm: [46] a notification, of the form defined by [46], of a specific event. An alarm may or may not represent an error. 
Alarm report: [46] a specific type of event report used to convey alarm information. Allomorphism: [50] the ability of a managed object of a given class to resemble the behaviour of one or more other managed object classes.

American National Standards Institute (ANSI): the principal standards setting body in the United States; ANSI represents the U.S. in ISO.

AMF: Alarm Reporting Function.

ANSI: American National Standards Institute.

AppleTalk: the Apple-defined protocol that allows transferring data between computers and peripherals via shielded twisted-pair wire at a 230-Kilobits-per-second rate.

ARF: Alarm Reporting Function.

ARR: Attributes for Representing Relationships.

ARPA: see Defense Advanced Research Projects Agency.

ASE: Application Service Element.

ASN.1: see Abstract Syntax Notation One. Associated objects: [58] managed objects, distinct from MOT(s), TO(s) and $\operatorname{TARR}(s)$, that are involved in a test. Asynchronous test: a test invocation for which the successful confirmation to the test request does not imply termination of the test invocation. 
Attribute: [38] a property of a managed object that is visible at the managed object boundary. Each attribute has an associated value which may have a simple or a complex structure; it may reflect or determine the behaviour of the managed object.

Attribute identifier: [50] an identifier used to distinguish an attribute of a managed object class from all other attributes.

Attribute type: [50] a named abstraci datatype used to specify the dataype of attributes. An attribute is an instance of an attribute type.

Attribute value assertion (AVA): [50] a proposition, which may be true, false, or undefined, concerning the value of an attribute.

Attribute value set: [52] a set of values, members of which are valid values of an attribute.

AVA: see Attribute value assertion

Backbone: the primary connectivity mechanism of a hierarchical distributed system. All systems which have connectivity to an intermediate system on the backbone are assured of connectivity to each other. Backup relationships: [45] an asymmetric relationship denoting that the second of a pair of managed objects (the backup object) is providing backup to the first (the backed up object). 
Baseband: a characteristic of any network technology that uses a single carrier frequency and requires all stations attached to the network to participate in every transmission.

Behaviour: [50] a description of the way in which managed objects, name bindings, attributes, notifications and actions interact with the actual resources they model and with each other.

BER: Basic Encoding Rules. Standard rules for encoding of arbitrary data structures described in ASN.1 and is defined in ISO 8825 [36] and [94].

Bridge: a network compontnt that functions at the media access control (MAC) sublayer of the OSI model's Data Link layer and is transparent to high-level protocols. Bridges can connect either local or remote networks and are capable of some isolation of network segments.

Broadband: a characteristic of any network that multipiexes multiple, independent network carriers onto a single cable.

Broadcast: a packet delivery system where a copy of a given packet is given to all hosts attached to the network.

Canadian Open Systems Application Criteria (COSAC): specifications that have been developed by the Treasury Board to provide guidance to departments and agencies of the federal government to assist them in complying with OSI implementation.

CCITT: see Comite Cunsultatif Internationale Telegraphique et Telephonique. 
CD: Committee Drafi.

Characteristics: [52] those elements of an object class definition that are inherited from a superclass or superclasses.

Class-specific elements: [52] those elements of an object class definition that are inherited from a superclass only if there are no conflicting inherited specializations and there is no further specialization of those elements in a subclass.

Client-server: a common way to describe network services and to model user processes (programs) of these services.

CMIP: see Common Management Information Protocol.

CMIS: see Common Management Information Service.

CMOT: see CMIP over TCPIIP.

CMIP over TCP/IP: a mapping of the OSI network management framework to management networks based on the Internet suite of protocols.

Comite Cunsultatif Internationale Telegraphique et Telephonique (CCITT): (International Telephone and Telegraph Consultative Committee) an agency of the United Nations comprising the national Post, Telephone and Telegraph (PTT) administrations.

Common Management Information Protocol: the OSI protocol for network management.

Common Management Information Service: the OSI service definition for network management. 
Conditional package: [50] a collection of optional attributes, notifications. operations and behaviour which are either all present or all absent in a managed object. The presence or absence of a package is conditional on the capability of the underlying resource (e.g. the options of an X.25 protocol machine).

Connectionless Network Protocol (CLNP): the OSI protocol for providing the OSI datagram service. CLNP is the OSI equivalent to Internet IP, and is sometimes called ISO IP.

Connectionless Transport Protocol (CLTP): the OSI protocol for providing endto-end data addressing and error control, but cannot guarantee delivery or provide flow control. The OSI equivalent of TCP/IP UDP.

Connectivity test: [62] the test used to verify that connectivity may be established between two entities (represented by an MOT and an Associated object) within a specified time.

Containment: [50] a structuring relationship for managed objects in which the existence of a managed object is dependsat upon the existence of a containing managed object. The contained managed object is said to be the subordinate object and the containing managed object the superior object.

Corporation for Open Systems (COS): a vendor and user group for conformance testing, certification and promotion of OSI products.

COSAC: see Canadian Open Systems Application Criteria.

DARPA: see Defense Advanced Research Projects Agency. 
Data integrity test: [62] the test to ascertain whether two entities can exchange data without any corruption, and to measure, where applicable, the time taken for the connection establishment.

Defense Advanced Research Projects Agency (DARPA): an agency of the U.S Department of Defense that sponsors high-risk, high-payoff research. DARPA was formerly known as ARPA when the ARPANET was built.

Diagnosis: the process in which one must determine those components of a system which, when assumed to be functioning abnormally, will explain the discrepancy between the observed and correct system behaviour.

DIS: Draft International Standard.

Discriminator: [47] a management support object that allows a system to control management operations and event reports relating to other managed objects.

Discriminator construct: [48] an attribute of the log which specifies tests on the information (potential log record) that is to be logged. It may operate on any of the parameters of the information to be logged.

Discriminator input object: [47] a conceptual object whose attributes are parameters of either a received MAPDU or a MAPDU that may be sent. Discriminator input objects are defined for the purpose of discrimination and instances of discriminator input objects exist only for the duration of discrimination. Some attributes have no matching rules defined for them, and so such attributes cannot be used for discrimination. 
Distinguished name: [50] the name of a managed object formed from the sequence of the RDN's of the managed object and each of its superior managed objects.

DMI: Definition of Management Information.

Encapsulation: [50] an enclosure relation between a managed object and its attributes, notifications, operations and behaviour. The enclosure relation assures that the managed object can maintain its integrity.

Entity: OSI terminology for a layer protocol machine.

ERF: Event Report Function.

Error: [46] a deviation of a system from normal operation. Errors are manifestations of a fault.

Ethernet: a Data Link protocol that uses baseband signaling at 10 or $16 \mathrm{Mbps}$ and is the predominant LAN standard.

Event: [24] a change in the status of a managed object, where the change meets predefined conditions which determine that notification of the change is necessary. Event forwarding discriminator: [47] a discriminator that acts on potential event reports.

Event report: [24] a report containing information about an event, such as the type of event, the status that changed, the probable cause and the effect of the event on the managed object. 
Event report management function (ERMF): [47] a function, including the definition of a management support object class, that allows a manager to control the transmission of event reports from managed objects independent of the definition of the managed objects.

Exterior Gateway Protocol (EGP): a reachability routing protocol used by gateways in a two-level internet. EGP is used in the Internet core system.

Failure: [98] the overall result of a fault. $A$ failure is the event of a system or component producing an error.

Fallback relationships: [45] an asymmetric relationship denoting that the second of a pair of managed objects (the secondary object) is capable of serving as a fallback or "next preferred choice" to the first managed object (the primary object).

Fault: [16] the physical or algorithm:c cause of a malfunction. Faults manifest themselves, directly or indirectly, as errors.

FDDI: see Fiber Distributed Data Interface.

Fiber Distributed Data Interface: a network based on the use of optical-fiber cable to transmit data at rates of $100 \mathrm{Mbps}$.

Functional Profile: a statement which identifies a group of standards together with appropriate options and parameters necessary to accomplish a function or set of functions.

FP: see Functional Profile. 
Gateway: a hardware or software device providing a communications link between a LAN and a WAN is a physically removed mainframe.

GDMO: Guidelines for the Definition of Managed Objects

GNMP: (U. S.) Government Network Management Profile

GOSIP: see Government OSI Profile.

Government OSI Profile: functional standards and profiles developed by national bolies of the U.S. and U.K. governments.

Group relationships: [45] a relationship between two managed objects where one. the member object, belongs to a group represented by the other, the owner object. HMI: Human Machine Interface

Hub: a device used to physically interconnect a series of computers in a common LAN.

IAB: see Internet Activities Board.

IEC: see International Electrotechnical Commission

IEEE: see Institute of Electrical and Electronic Engineers.

ETF: see Internet Engineering Task Force.

Information: [94] (in the protocol sense) data with associated meaning. Information object: [94] a piece of design which requires to be identified unambiguously in some instance of communication.

Inheritance: [50] the conceptual mechanism by which attributes, notifications, operations and behaviour are acquired by a subclass from its superclass. 
Inheritance hierarchy: [50] a hierarchical arrangement of managed object classes where the hicrarchy is organised on the basis of the class specialization. A subclass is a managed object class that is a specialization: of another managed object class and is lower in the hierarchy than the class of which it is a specialization. Institute of Electrical and Electronic Engineers: a profession organization, which, as part of its services to the community, performs some pre-standardization work for ISO.

Internal resource test: [62] the test used to specify the execution of test mechanisms which exercise the function of a resource which is internal to the system under test and to report the result of the exercise. Such test mechanisms are somtimes known as self tests. The nature of the resource(s) under test and the exercises is specific to the containing system.

International Electrotechnical Commission (IEC): an organization that produces much of the world's standards, commonly referred to as IEC.

International Organization for Standardization (ISO): an organization that produces much of the world's standards, commonly referred to as $1 S O$.

International Standardized Profile (ISP): an intemationally agreed to, harmonized document which identifies a standard or group of standards, together with options and parameters necessary to accomplish a function or set of functions.

International Standards Organization: see International Organization for Standardization. 
Internationa! Telephone and Telegraph Consultative Committee (CCITT): a body of the International Telecommunications Union (ITU) comprising the national Post, Telephone and Telegraph (PTT) administrations and an agency of the United Nations. commonly referred to as CCITT.

Internet: a large collection of connected networks, primarily in the United States, running the Internet suite of protocols. Sometimes referred to as the DARPA Internet. NSFIDARPA Internet, or the Federal Research Internet.

Internet Activities Board: the technical body overseeing the development of the Internet suite of protocols. The IAB consists of several task forces, each charged with investigating a particular area.

Internet Control Message Protocol (ICMP): the protocol used to handle errors and control messages at the Internet IP layer. ICMP is actually part of the IP protocol. Internet Engineering Task Force: a task force of the Internet Activities Board charged with solving the short-term needs of the Intemet. Internet Gateway Protocol (IGP): the protocol used to exchange routing information between collaborating routers in the Internet.

Internet Protocol (IP): the network protocol offering connectionless-mode network service in the Internet suite of protocols.

Internet Research Task Force: a task force of the Intemet Activities Board charged with research and development of the Internet. 
Internet suite of protocols: a collection of computer-communication protocols originally developed under DARPA.

Intrusive test: [58] a statement with respect to a test invocation if service user disruption will or may occur as a result of the test.

IRTF: see Internet Research Task Force.

ISO: see International Organization for Standardization

ISODE (ISO Development Environment): a popular implementation of the upper izyers of MSI over the lower layers of TCP/P (see Chapter 6 (6.2.4)).

ISO/IEC JTC1: the combined committee, Joint Technical Committee 1, of these two international standards bodies. Within ISO/IEC JTCl, Working Group 4 of Subcommittee 21 (SC21/WG4) is responsible for OSI Management.

LAN: see Local Area Network.

\section{LCF: Log Control Function.}

Local Area Network: any one of a number of communications technologies providing high speed, low latency data transfer over a small geographic area. Log: [48] a management support object class that models resources used as a repository for log records.

Log record identifier: [48] a number, with the syntax of an integer, that is unique within the scope of the $\log$ and is assigned sequentially.

Loyging time: [48] the time at which the record was entered into the log. 
Loopback test: [62] the test used to verify that data may be sent and received over a specified communications path with an acceptable error rate.

MAC: see Media Access Control.

Managed object (MO): [37] the OSI Management view of a resource within the OSI Environment that may be managed through the use of OSI Management protocol(s), such as a layer entity, a connection or an item of physical communications equipment.

Managed object boundary: [50] the conceptual location where aspects of an underlying resource are made visible to management and which bounds the scope of the managed object's definition.

Managed object class: [42] a named set of managed objects sharing the same set of attributes, notifications and management operations.

Managed object class definition: [52] a set of attribute, operation, notification and behaviour definitions to which a managed object class name has been allocated, documented by the use of template(s). The definition of a managed object class includes all elements of definition inherited from the superclass(es) of the managed object class and all elements of definition that constitute specialization(s) of the superclass(es).

Management domain: [42] a set of managed objects, collected for systems management for which a common policy applies. 
Management information: [42] the information within an open system which may be transferred or affected through the use of OSI management protocols.

Management Information Base: [37] the conceptual repository of management information within an open system.

Management interaction: [42] a single management operation or a single notifi cation or an identified set of logically related management operations and notificationsduring which the manager and agent roles do not change. Management support object: [42] a system object defined specifically to support a systems management function (e.g. log, discriminator).

Manager: [42] an MIS-user (application) which for a particular exchange of systems management information has taken a manager role.

Manager role: [42] an MIS-user (application) taking a manager role is capable of issuing management operations and of receiving notifications.

Media Accss Control (MAC): a lower sublayer of the Data Link layer of the OSI model. The MAC layer supports medium-dependent functions.

MHS: Message Handling Systems (e.g. X.400).

MIB: see Management Information Base.

MIL: Management Information Library.

MIM: Management Information Model.

MIS-user: [42] an application making use of system management services.

MOC: see Management Object Class. 
MOT: [58] the attribute which identifies the managed object instance(s) which represents a management view of the resource or resources whose functionality is the subject of a test.

MSF: Measurement Summarization Function.

Multiple inheritance: [50] a conceptual mechanism that allows a subclass to acquire its attributes, notifications, operations and behaviour from more than one superclass.

MUX: Multiplexor.

Name binding: [50] a relation between managed object classes for the purpose of naming. see also Naming tree.

Naming schema: [50] a collection of name bindings.

Naming tree: [50] a hierarchical arrangement of managed objects where the hierarchy is organized on the basis of the containment relationship. A managed object used to name another managed object is higher in the hierarchy than the named object. The naming object is referred to as being the superior of the named object, which is referred to as the subordinate.

Network Management: the overseeing and maintaining of a network. The activities of network management include configuring the network, monitoring performance, maintaining security and fault detection and correction.

(N)-layer managed object: [42] a managed object specific to the (N)-layer. 
(N)-layer operation: [37] the monitoring and control of a single instance of communication.

NM: see Network Management.

NMSIG: Network Management Special Interest Group.

Node: a point in a network $\therefore$ here service is provided, service is used, or communications channels are interconnected.

Non-intrusive test: [58] a statement with respect to a test invocation if no service user disruption will or may occur as a result of the test.

Notification: [42] information emitted by a managed object relating to an event that has occured within the managed object.

Notification type: [42] a named datatype defining a specific kind of notification.

Object descriptor type: [94] a useful ASN.1 type whose values are textual (humanreadable) identifiers of information objects.

OID (Object identifier): a leaf instance of OIT.

Object identifier tree (OTT): [94] a tree formed for the purpose of naming information objects.

Object identifier type: [94] a built-in ASN.1 typ: whose values are the names of the nodes of the object identifier tree.

: [50] the principle of defining objects, where an object is an abstraction of a physical or logical thing.

OIT: see Object Identifier Tree 
OIW: OSI Implementors Workshop.

OMF: Object Management Function.

Open system: the representation within the Basic Reference Model [34] of those aspects of a real open system that are pertinent to OSI.

Open Systems Interconnection: an international effort to facilitate communications among computers of different manufacture and technology.

Operation: [42] an operation on a managed object to effect systems management.

OSI: see Open Systems Interconnection.

OSI Management: [37] the facilities to control, coordinate and monitor the resources which allow communications to take place in the OSI Environment (i.e. all layers of the OSI architecture).

OSI model: the Basic Reference Model specified by ISO 7498 [34]. The model divides a complex set of communications functions into seven self contained layers or modules.

Parameter: [52] a value which may be carried in protocol but which is defined as part of a managed object class definition.

PBX: Private Branch Exchange.

Peer relationships: [45] a symmetric relationship describing the rules under which pairs of similar managed objects communicate. 
Ping: a UNIX process which utilizes the ICMP (TCP/IP) protocol's mandatory ECHO_REQUEST datagram to elicit an ICMP ECHO_RESPONSE from a specified host or network gateway.

Potential event report: [47] a type of a discriminator input object that is defined for the purpose of event forwarding discrimination. A potential event report consists of all the information required to be forwarded in the event report. The information is derived from the information contained in the notification and information derived from local processing of the notification.

Potential log record: [48] a management support object class that models units of information stored in a log.

Protocol: the definition of standards to be observed, and a set of rules that define procedures and message format to allow devices on a network to communicate. Protocol integrity test: [62] the test to enable the control of the generation of PDUs from the MOT identified by the test request. The order and temporal interval between PDUs may be defined. Actual responses $\pi$ ay be compared with expected responses. Proxy: the mechanism whereby one system "fronts for" another system in responding to protocol requests. Proxy systems are used in network management to avoid having to implement full protocol stacks in simple devices, such as modems. RDN: see Relative distinguished name. Real open system: [34] a real system which complies with the requirements of OSI standards in its communication with other real systems. 
Relational database: a method of storing information in which the relationship between different fields in a record is made explicit so that data can be updated and searched in terms of those relationships instead of field locations.

Relative distinguished name (RDN): [50] an attribute value assertion, which is true, concerning the attribute used in naming.

Repeater: a device which propagates electrical signals from one cable to another without making routing decisions cr providing packet filtering. In OSI terminology, a repeater is a Physical Layer inte mediate system.

Request for Comments: the jocument series describing the Internet suite of protocols and related experiments.

RFC: see Request for Comments.

Router: a network component that functions at the Network layer of the OSI model to forward packets in accordance with the methods described by high-level protocols. Routers can connect either local or remote networks and can create many logically different subnetworks within an internetwork.

Service relationships: [45] an asymmetric relationship denoting that the first of a pair of managed objects is a provider object (providing services) to the second and that the second is a user object (using services) of the first.

SF: Summarization Function.

SGMP (Simple Gateway Management Protocol): the predecessor to SNMP. SIG: Special Interest Group. 
SMAE: System Management Application Entity.

SMF: see System Management Function

SMFA: Systems Management Functional Area.

SMI (Structure of Management Information): the rules used to define the objects that can be accessed via a network management protocol.

SMO: System Management Overview.

SNMP (Simple Network Management Protocol): the network management protocol of choice for TCP/IP internets.

Specialization: [50] the technique of deriving new managed object classes from an existing class by the addition of new capabilities (such as new attributes or notifications).

Status: [24] a measurement of the behaviour of a managed object at a specific instance in time. Status is represented by a set of status attributes and their assigned values at the specific time.

STMF: State Management Function.

Subclass: [50] a class derived from another class by specialization. see Inheritance hierarchy.

Subnetwork: a portion of a network that is partitioned by a router. Subordinate object: [50] see see Naming tree.

Superclass: [50] a class used in deriving another class by specialization. see Inheritance hierarchy. 
Superior object: [50] see Naming tree.

Synchronous test: [58] a test invocation for which any confirmation to the test request implies termination of the test invocation.

Systems management application entity (SMAE): an application-entity for the purpose of systems management communication.

Systems Management Function (SMF): [42] a part of systems management activities which satisfy a set of logically related user requirements.

Systems Management Functional Area (SMFA): [42] a category of systems management user requirements.

Systems management protocol: [42] an application layer protocol supporting systems management services.

Systems management service : [42] a named set of service primitives that provide a service for use in systems management

TARR: see Test action request receiver TCP: see Transmission Control Protocol

TCP/IP: a set of de facto networking standards commonly used over Ethernet or X.25 networks to tie computers and data communications equipment into computer networks. see Transmission Control Protocol and Internet Protocol.

Template: [52] a standard format for the documentation of managed object class definitions, conditional packages, parameters, name bindings, attributes, group attributes, behaviour definitions, actions or notifications. 
Test action request receiver (TARR): [58] a term used to identify the ability of a managed object to act upon a test request. In addition to those required as a TARR, the managed object may also have other attributes, operations and notifications. A managed object with TARR functionality may create (and subsequently delete) an instance of a particular TO class (as the result of receiving a test request), representing a specific test invocation.

Test conductor (TC): [58] a manager that issues test operations.

Test invocation: [58] a specific instance of test, from the time of invocation to termination.

Test object (TO): [58] a managed object that exists only for the duration of a test invocation and which has attributes, operations and notifications that pertain to that instance of test.

Test performer: [58] an agent which receives test operations.

Test request: [58] an individual request, in the form of an action operation issued by the test conductor to a test performer, in order to initiate one or more test invocations.

Test session: [58] a set of test invocations.

TMF: Test Management Function.

TO: see Test object

Topeiogy: the pattem of physical interconnections between devices on the network. TP: Transaction Processing. 
Transmission Control Protocol (TCP): the transport protocol offering a connectionoriented transport service in the Internet suite of protocols.

UDP: see User Datagram Protocol

User Datagram Protocol (UDP): a transport protocol in the Internet suite of protocols. UDP, like TCP, uses IP for delivery: however, unlike TCP, UDP provides for exchange of datagrams without acknowledgments or guaranteed service.

VT: Virtual Terminal.

WAN: see Wide Area Network.

WD: Working Document.

Wide Area Network: any number of technologies providing geographically distant transfer.

WMF: Workload Monitoring Function. 


\section{Appendix B June 1991 ISO/IEC Developments}

This Appendix lists the change in status of the ISO Network Management standards as a result of ISO meeting in Arles, France in June 1991.

\section{Highlights of Changes to SMI Part 1 (10165-1) [MM]:}

- 1.1 SMI Part I was progressed to IS by a unanimous vote.

- $\quad 1.2$ The concept of compatibility have been introduced.

- Compatibility is introduced as a collection of rules and restrictions imposed on inheritance in order to assure interoperability. Strict inheritance is defined to follow these compatibility rules and is the form of inheritance supported by SMI Part 1. (In essence the rules are the same as those previously required for allomorphic inheritance.) Compatibility is a relationship between a managed object (instance) (referred to as the extended managed object) and a managed object class (referred to as the compatible class).

- 1.3 Two methods of providing interoperability have been defined:

- 1.3.1 Agent provided interoperability:

- This capability is provided by means of allomorphism. Allomorphism is now a relationship between an extended managed object and the allomorphic classes. (This is a result of the above compatibility definitions). Allomorphic classes and the managed object class of the extended managed object do not have to be related via inheritance; they must only satisfy the compatibility requirements.

- 1.3.2 Manager provided interoperability: 
- This capability is provided by the managers ability to recognize familiar information and ignore unknown information. This capability is enhanced if the managed objects the manager is trying to manage are compatible with managed object classes the manager knows.

- 1.4 The IS supports local and global naming. Naming is supported by vising either the systemld attribute as part of the distinguished name ur by use of the systemTitle attribute. The latter may be an OBJECT IDENTIFIER. No further details on the use of these attributes is defined in the IS.

- 1.5 Attributes in TOP have changed (see Attachment).

\section{Highlights of Changes to SMI Part $4(10165-4)$ [GDMO]}

- 2.1 GDMO was progressed to IS with Denmark voting NO.

- 2.2 GDMO and MIM are now aligned:

- 2.2.1 Permitted and Required attribute value sets are now defined and supported in Part 1.

- 2.2.2 CREATE/DELETE semantics are in the name binding.

- 2.2.3 The PARAMETER template must have a SYNTAX clause, thus, restricting its use to resolving ANY DEFINED BYs.

- 2.2.4 Minor changes have been made to the syntax of some templates; e.g. BEHAVIOUR clauses must be "escaped" by the use of double quotes ["].

- 2.2.5 The OBJECT IDENTIFIER usage remains the same as in the DIS. 
3 Highlights of Changes to SMO (10040)

- 3.1 SMO was progressed to IS with Denmark voting NO.

- 3.2 For function and CMIP conformance General and Dependent conformance classes have been defined. General conformance implies unrestricted to conformance to all elements cf procedure specified in the base standard. Dependent conformance implies conformance for a particular standardized use of the services provided by the base standard: e.g. ISPs.

- 3.3 Conformance is defined for function protocols, managed objects and communications protocols.

- 3.4 Managed Object definers may specify consistency requirements between various standardized ways of perceiving the same resource.

- 3.5 Only one management application context has been defined; it is the combined manager/agent context. Role negotiation is available through functional units of SMFs.

\section{Highlights of Changes to 10164-1 [Object Management Function]}

- 4.1 10164-1 was progressed to IS unanimously.

- 4.2 Pass-through services are still there.

- 4.3 Functional Units have been defined for:
a. Monitor
b. Control
c. All Events 
d. Object Management Events

\section{Highlights of Changes to 10164-2 [State Management Function]}

- 5.1 10164-2 was progressed to IS unanimously.

- 5.2 Most "bugs" seem to be fixed; i.e. dependencies between various state and status attributes have been relaxed. (E.g., The Off-Duty availability status is no longer coupled to the Disabled operational state).

- 5.3 A standby starus and unknown status have been added. (Standby has the values Hot and Cold Standby). Installation status has been renamed Procedural status. Repair status has been renamed Alarm status. Values have been added to accommodate testing, e.g. reporting test results, terminating test, etc. Some renaming of status attribute values was also done.

- $\quad 5.4$ State management has one functional unit for State Change Events

\section{Highlights of Changes to 10164-3 [Relationship Management Attributes]}

- 6.1 10164-3 was progessed to IS unanimously.

- 6.2 No significant changes were made; the backup operation, as proposed by Japan, was not included.

- 6.3 One functional unit is defined in this document for Relationship Change Events.

7 Highlights of Changes to 10164-4 [Alarm Reporting Function]

- 7.1 10164-4 was progressed to IS . 
- 7.2 No significant changes; probable cause supported in both Object Identifier and integer encoding. Rationalization of the semantics and assignment of probable causes.

- 7.3 One functional unit for Alarm Reporting.

\section{Highlights of Changes to 10164-5 [Event Report Management Function]}

- 8.1 10164-5 was progressed to IS.

- 8.2 MODE indicator added to discriminator (part of a conditional package) to allow sending of event reports in either confirmed or unconfirmed mode.

- 8.3 Discriminator Id is not include in event reforts.

- 8.4 Local events are logged directly without going through event forwarding discriminator.

- 8.5 Two functional units defined, monitor and control.

\section{Highlights of Changes to 10164-ó [Log Control Function]}

- 9.1 10164-6 was progressed to IS.

- $\quad 9.2 \mathrm{Log}$ is not a subclass of discriminator.

- 9.3 Local events are logged directly without going through an event forwarding discriminator.

- 9.4 Two functional units denned, monitor and control.

10 Highlights of Changes to 10164-7 [Security Alarm Report Function]

- 10.1 10164-7 was progressed to IS unanimously.

- 10.2 No significant change from DIS. 
- 10.3 One functional unit defined, Security Alarm Reponit.:

\section{Miscellaneous}

- 11.1 CMIP PICS went to CD.

- $\quad 11.2$ Accounting Meter went to 2nd CD.

- 11.3 Scheduling and Time Management Functions did not advance.

- 11.4 Domains is a working draft within the Architecture Group.

- 11.5 First Working Document on Software Management produced.

- 11.6 Test Class work advanced for loopback tests. Possible CD date 12/91. 


\section{Appendix C Previous Implementations}

BIBLIOGRAPHY In [88], Sethi presents a biblicgraphy of nearly 200 articles on network management. Some of the major topics covered include Fault Management and Diagnosis, Al Applications in Network Management, LAN Management and Internet Management.

Integrated Network Management, I (Proceedings of the IFIP TC 6/WG 6.6 Symposium on Integrated Network Management) [75] contains 46 refereed papers and 7 invited papers on management policy, administration, and operation of local and wide area communication networks, including data, voice, and integrated communications.

Integrated Network Management, II (Proceedings of the IFIP TC 6/WG 6.6 Second International Symposium on Integrated Network Management) [66] contains 50 refereed papers and 9 invited papers on the administration, operation, maintenance, and security of local and wide area communication networks, including data, voice, and video communications.

GENERAL In [54], Joseph et al describe the MAP Fault Management system (MAPFaM) which is an evolving expert system for fault management of MAP and other OSI networks.

In [100], Yamahira et al describe the processing configuration of EXNETS (EXpert system for NEtwork TroubleShooting), a network fault management expert system used to detect, diagnose, and recover from computer network malfunctions. 
APPLYNG ARTIFICIAL INTELLIGENCE (AI) In [30], Goyal presents examples of technologies in three key areas of Al from the standpoint of telecommunication network operation and management: distributed $\mathrm{AI}$, knowledge representation, and machine learning. This paper is an attempt to evaluate how these technologies might aid in automating the network management tasks of evolving future networks.

EXPERT SYSTEMS Erickson et al [23] present a collection of early papers on the application of expert system technology to the integrated network management problem.

The Integrated Network Management System (INMS) [29] presented by Goldman et al consists of several interacting rule-based expert systems and incorporates the network structure information in the form of a relational database.

In [31], Hibino presents an object-oriented troubleshooting expert system for electronic switching systems.

Searls et al [87] present two examples of managing flow control for modeling the actual procedures of the domain expert.

In [86], Schnaidt and Reddy of Carnegie Mellon University describe an innovative topology (a backbone-in-a-box) coupled with a sophisticated management system (a practical expert system).

In [25], Feridun describes The Connectivity Tool, a system designed to diagnose end-to-end connectivity problems in the Internet.

MODEL-BASED REASONING Fulton [28] proposes that model-based expert systems can perform effective diagnosis for applications where a complete and accurate 
model of the system can be created. Model-based sysierns contain a model simulating the structure and function of the system under diagnosis. In diagnosing a failure, the model-based system reasons about what could be wrong with the simulated model to yield the same results as the observed system.

BLACKBOARD Buttner et al [7] present an initial experiment with model-based reasoning in a blackboard environment. The power of the blackboard-based system arises from the flexibility provided in adding new models of diagnosis. Frontini et al [27] describe a knowledge-based system, using a blackboard architecture, for monitoring and fault localization in wide area networks.

NEURAL NETWORKS In [12], Cebulka et al consider potential applications of neural networks for tasks in network management. Covo et al [18] describe a centralized network management prototype consisting of neural networks in cooperation with a rulebased expert system. The multiple neural networks perform anomaly detection and isolation.

NON-DETERMINISTIC REASONING In [32], Hong and Sen examine the complexities of managing a heterogeneous enterprise network by employing a network uncertainty model with a network belief language.

ORDER OF MAGNITUDE REASONING In [19], Dague shows how order of magnitude reasoning has been successfully used for troubleshooting complex analog circuits. The originality of this approach was to be able to remove the gap between the informa- 
tion required to apply' a general theory of diagnosis and the limited information actually available.

AUTOMATIC ERROR RECOVERY In [17], Cochran and Mellichamp describe a system called AUTOREC (automatic error recovery) to do error detection and recovery on a packet switching network. 


\section{Bibliography}

[1] Elizabeth K. Adams. Global commonality in user requirements. In I. Krishan and W. Zimmer, editors, Integrated Network Management, II, pages 171-181. Elsevier Science Publishers B.V.(North-Holland), April 1991.

[2] Subodh Bapat. Osi management information base implementation. In I. Krishan and W. Zimmer, editors, Integrated Network Management, II, pages 817-831. Elsevier Science Publishers B.V.(North-Holland), April 1991.

[3] G. Bochmann and P. Mondain-Monval. Formal description of network management issues. Technical Report 734, Universite de Montreal, July 1990.

[4] G. v. Bochmann and M. Deslauriers. Combining asn.1 support with the lotos language. In M. Diaz, editor, Protocol Specification, Testing, and Verification, IX. Elsevier Science Publishers B.V.(North-Holland), 1990.

[5] Grady Booch. Object Oriented Design with Applications. Benjamin/Cummings Publishing, 1991.

16] Paul Brusil and Daniel Stokesberry. Integrated network management. In B. Meandzija and J. Wescott, editors, Integrated Network Management, I, pages 3-9. Elsevier Science Publishers B.V.(North-Holland), May 1989.

[7] K. Buttner, D. Sriram, and M. Freiling. An object-oriented blackboard architecture for model-based reasoning. In Blackboard Architectures and Applications, pages 403431. Academic Press, Inc., 1989. 
[8] J. Case, J. Davin, M. Fedor, and M. Schoffstall. A simple gateway monitoring protocol. Technical Report RFC 1028, November 1987.

[9] J. Case, M. Fedor, M. Schoffstall, and J. Davin. A simple network management protocol (snmp). Technical Report RFC 1098, April 1989.

[10]J. Case, M. Fedor, M. Schoffstall, and J. Davin. A simple network management protocol (snmp). Technical Report RFC 1157, May 1990.

[11]L. Cassel, C. Partridge, and J. Westcott. Network management architectures and protocols: Problems and approaches. IEEE Journal on Selected Areas in Communications, 7(7):1104-1114, September 1989.

[12]K. D. Cebulka, M. J. Muller, and C. A. Riley. Applications of artificial intelligence for meeting network management challenges in the 1990's. In IEEE Global Telecommunications Conference and Exhibition, volume 1, pages 501-506. IEEE, Nov 1989.

[13]V. G. Cerf. lab recommendations for the development of internet network management standards. Technical Report RFC 1052, April 1988.

[14]V. G. Cerf. Report of the second ad hoc network management review group. Technical Report RFC 1109, August 1989.

[15]V. G. Cerf. Internet activities board. Technical Report RFC 1160, May 1990. [Obsoletes RFC 1120].

[16]P. Chen. The entity-relationship model-toward a unified view of data. $A C M$ Transactions on Database Systems, 1(1):9-36, March 1976. 
[17]Teresa Cochran and Joseph M. Mellichamp. Autorec:an automated error recovery system for network management. IEEE Network, pages 14-18, March 1990.

[18]A. Covo, T. Moruzzi, and E. Peterson. Ai-assisted telecommunications network management. In IEEE Global Telecommunications Conferente and Exhibition, volume 1, pages 487-491. IEEE, Nov 1989.

[19]Philippe Dague and Olivier Raiman. Troubleshooting:when modeling is the problem. In Proceedings of AAAl-87, pages 600-605. AAAI, 1987.

[20]R. P. Davidson and N. J. Muller. LANs to WANs. Artech House, Inc., 1990.

[21]N.W. Dawes. Pegasus - general architecture and designs. Technical Report SCE-9104, Systems and Computer Engineering, Carleton University, February 1991.

[22]H. Ehrig and B. Mahr. Fundamentals of Algebraic Specification. Springer-Verlag, 1985.

[23]Eric Ericson. General network management applications. In Eric Ericson, Lisa Traeger Ericson, and Daniel Minoli, editors, Expert Systems Applications in Integrated Network Management, pages 71-75. Artech House, Inc., 1989.

[24]Lev Feldkhun and John Erickson. Event management as a common functional area of open systems management. In B. Meandzija and J. Wescott, editors, Integrated Network Management, I, pages 365-376. Elsevier Science Publishers B.V.(NorthHolland), May 1989.

[25]Metin Feridun. Diagnosis of connectivity problems in the internet. In I. Krishan and W. Zimmer, editors, Integrated Network Management, II, pages 691-701. Elsevier 
Science Publishers B.V.(North-Holland), April 1991.

[26]Sharon Fisher. Dueling protocols. BYTE, pages 183-190, March 1991.

[27]M. Frontini, J. Griffin, and S. Towers. A knowledge-based system for fault Incalisation in wide area networks. In I. Krishan and W. Zimmer, editors, Integrated Netw'ork Management, II, pages 519-530. Elsevier Science Publishers B.V.(North-Holland), April 1991.

[28]Steven L. Fulton and Charles O. Pepe. An introduction to model-based reasoning. Al Expert, pages 48-55, January 1990.

[29]J. Goldman, P. Hong, C. Jeromnimon, G. Louit, J. Min, and P. Sen. Integrated fault management in interconnected networks. In B. Meandzija and J. Wescott, editors, Integrated Network Management, I, pages 333-344. Elsevier Science Publishers B.V.(North-Holland), May 1989.

[30]Shri K. Goyal. Knowledge technologies for evolving networks. In I. Krishan and W. Zimmer, editors, Integrated Network Management, II, pages 439-461. Elsevier Science Publishers B.V.(North-Holland), April 1991.

[31]Yuuji Hibino and Kisaku Fujimoto. An object-oriented troubleshooting expert system for electronic switching systems. In IEEE Global Telecommunications Conference and Exhibition, volume 1, pages 475-479. IEEE, Nov 1989.

[32]Paul Hong and Prodip Sen. Incorporating non-deterministic reasoning in managing heterogeneous network faults. In I. Krishan and W. Zimmer, editors, Integrated Network Management, II, pages 481-492. Elsevier Science Publishers B.V.(NorthHolland), April 1991. 
[33]DARPA Internet Activities Board. Iab official protocol standards. Technical Report RFC 1200, April 1991. [Obsoletes RFC 1140].

[34]ISO/IEC. ISOIIEC 7498, Information Processing Systems - Open Systems Interconnection - Basic Reference Model, 1984.

[35]ISO/IEC. ISOIIEC 8824, Information Processing Systems - Open Systems Interconnection - Specification of Abstract Syntax Notation One (ASN.I), December 1987.

[36]ISO/IEC. ISOIIEC 8825, Information Processing Systems - Open System Interconnection - Specification of Basic Encoding Rules for ASN.I, Dec 1987.

[37]ISO/IEC. ISOIIEC 7498-4, Information Processing Systems - Open Systems Interconnection - Basic Reference Model - Part 4: Management Framework, 1989.

[38]ISO/LEC. ISOIIEC 9595, Information Processing Systems - Open Systems Interconnection - Information Service Definition - Common Management Information Service Definition, December 1989.

[39]ISO/EC. ISOIIEC 9596, Information Processing Systems - Open Systems Interconnection - Information Protocol Definition - Common Management Information Protocol Specification, December 1989.

[40]ISO/IEC. ISOIIEC DIS I0165-3, Information Technology - Open Systems Interconnection - Management Information Services - Structure of Management Information Part 3: Definition of Managed Attributes, 1989. [Obsoleted by inclusion in 10165-2].

[41]ISO/IEC. ISOIIEC 9594-I, Information Technology - Open Systems Interconnection - Directory, June 1990. 
[42]ISO/IEC. ISOIIEC DIS 10040. Information Processing Systems - Open System Interconnection - Systems Management Overview, June 1990.

[43]ISO/IEC. ISOIIEC DIS 10164-1, Information Technology - Open Systems Interconnection - System management - Part 1: Object Manajement Function, June 1990.

[44]ISO/IEC. ISOIIEC DIS 10164-2, Information Technology - Open Systems Interconnection - System management - Part 2: State Management Function, June 1990.

[45]ISO/EC. ISOIIEC DIS 10I64-3, Information Technology - Open Systems Interconnection - System management - Part 3: Relationship Management Function, June 1990.

[46]ISO/IEC. ISOIIEC DIS 10164-4, Information Technology - Open Systems Interconnection - System management - Part 4: Alarm Reporting Function, June 1990.

[47]ISO/IEC. ISOIIEC DIS 10164-5, Information Technology - Open Systems Interconnection - System management - Part 5: Event Report Management Function, June 1990.

[48]ISO/IEC. ISOIIEC DIS 10164-6, Information Technology - Open Systems Interconnection - System management - Part 6: Log Control Function, June 1990.

[49]ISO/IEC. ISOIIEC DIS 10164-7, Information Technology - Open Systems Interconnection - System management - Part 7: Security Alarm Reporting Function, June 1990.

[50]ISO/IEC. ISOIIEC DIS 10165-I, Information Technology - Open Systems Interconnection - Management Information Services - Structure of Management Information Part 1: Management Information Model, June 1990.

[51]ISO/IEC. ISOIIEC DIS 10165-2, Information Technology - Open Systems Intercon- 
nection - Management Information Services - Structure of Management Information Part 2: Definition of Management Information, June 1990.

[52]ISO/IEC. ISOIIEC DIS 10]65-4, Information Technology - Open Systems Interconnection - Management Information Services - Structure of Management Information Part 4: Guidelines for Definition of Managed Objects, June 1990.

[53]ISO/LC. ISOIIEC DIS 9834, Information Technology - Open Systems Interconnection - Procedures for the Operation of OSI Registration Authorities, 1990.

[54]C. Joseph, J. Kindrick, K. Muralidhar, C. So, and T. Toth-Fejel. Map fault management expert system. In B. Meandzija and J. Wescoth, editors, Integrated Network Management, I, pages 627-636. Elsevier Science Publishers B.V.(NorthHolland), May 1989.

[55]ISO/EC JTC1/SC21. ISOIIEC JTC1/SC21 N4077, Information Processing Systems - Open Systems Interconnection - System management - Fault Management Working Document, November 1989.

[56]ISO/IEC JTC1/SC21. ISOIIEC CD 10164-10, Information Technology - Open Systems Interconnection - System management - Part 10: Accounting Meter Function, July 1990. ISO/EC JTCI/SC21 N4958.

[57]1SO/EC JTC1/SC21. ISOIIEC CD 10164-11, Information Technology - Open Systems Interconnection - System management - Part 11: Workload Monitoring Function, June 1990. ISO/EC JTC1/SC21 N4959.

[58]ISO/IEC JTC1/SC21. ISOIIEC CD 10I64-12, Information Technology - Open Systems Interconnection - System management - Part 12: Test Management Function, 
December 1990. ISO/IEC JTC1/SC21 N5517.

[59]ISO/IEC JTC1/SC21. ISOIIEC CD 10164-8, Information Technology - Open Systems Interconnection - System management - Part 8: Security Audit Trail Function. June 1990. ISO/IEC JTC1/SC21 N4955.

[60]ISO/IEC JTC1/SC21. ISOIIEC CD 10164-9, Information Technology - Open Systemus Interconnection - System management - Part 9: Objects and Attributes for Access Control, June 1990. ISO/IEC JTC1/SC21 N4956.

[61]ISO/IEC JTC1/SC21. ISOIIEC CD I0I64-]3, Information Technology - Open Systems Interconnection - System management - Part J3: Summarization Function, January 1991. ISO/IEC JTC1/SC21 N5519.

[62]ISO/IEC JTC1/SC21/WG4. ISOIIEC JTCI/SC2I N5518, Information Technology - Open Systems Interconnection - System management - Part z: Confidence and Diagnostic Test Classes, December 1990.

[63]S.O. Kesti and K.T. Onka. Use and applicability of asn.1. In J. de Meer, L. Mackert, and W. Effelsberg, editors, Protocol Test Systems, pages 39-50. Elsevier Science Publishers B.V.(North-Holland), 1990.

[64]G. Knight, G. Pavlou, and S Walton. Experience of implementing osi management tacilities. In I. Krishan and W. Zimmer, editors, Integrated Network Management, II, pages 259-270. Elsevier Science Publishers B.V.(North-Holland), April 1991.

[65]Yoshikazu Kobayashi. Integrated network management. In B. Meandzija and J. Wescott, editors, Integrated Network Management, I, pages 79-90. Elsevier Science Publishers B.V.(North-Holland), May 1989. 
[66]I. Krishan and W. Zimmer. Integrated Network Management, II. Elsevier Science Publishers B.V.(North-Holland), April 1991.

[67]L. LaBarre. Osi internet management: Management information base. Technical Report RFC 1214, April 1991.

[68]C. Manson and J. S. Haugdahl. Dynamic and distributed. BYTE, pages 167-172, March 1991.

[69]Larry J. Marks and Eliabeth K. Adams. Network management-the road to standards. UNIXWOKLD, pages 99-100, February 1990.

[70]K. McCloghrie and M. Rose. Management information base for network managernent of tcp/ip-based internets. Technical Report RFC 1066, August 1988.

[71]K. McCloghrie and M. Rose. Structure and identification of management information for tcp/ip-based internets. Technical Report RFC 1065, August 1988.

[72]K. McCloghrie and M. Rose. Management information base for network management of tcp/ip-based intemets. Technical Report RFC 1156, May 1990.

[73]K. McCloghrie and M. Rose. Structure and identification of management information for tcp/ip-based internets. Technical Report RFC 1155, May 1990.

[74]K. McCloghrie and M. Rose. Management information base for network management of tcp/ip-based intemets:mib ii. Technical Report RFC 1213, March 1991. [Obsoletes RFC 1158].

[75]B. Meandzija and J. Wescott. Integrated Network Management, I. Elsevier Science Publishers B.V.(North-Holland), May 1989. 
[76]B. N. Meandzija, K. W. Kappel, and P. J. Brusil. Integrated network management and the international symposia. In I. Krislıan and W. Zimmer, editurs, Integrated Netw'ork Management, II, pages 3-6. Elsevier Science Publishers B.V.(North-Holland), April 1991.

[77]Tom Morocz. Six-month payback. LAN Magazine, pages 83-91, March 1991.

[78]NIST/OIW NMSIG. Stable implementation agreements for osi protocols: Part 13 network management. Technical report, March 1991.

[79]NIST/OIW NMSIG. Working implementation agreements for osi protocols part 18 network management. Technical report, March 1991.

[80]National Institute of Standards and Technology. Proposed government network management profile. Technical Repont version 1, U.S. Government, May 1991.

[81]Craig Partridge and Keith McCloghrie. Network management in the tcp/ip protocol suite. In William Stallings, editor, Handbook of Computer-Communications Standards, Volume 3, Second Edition. Howard W. Sams and Company, 1989.

[82]Craig Partridge and Glenn Trewitt. The high-level entity management system (hems). IEEE Network, pages 37-42, March 1988.

[83]Marshali T. Rose. The Open Book: A Practical Perspective on OSI. Prentice Hall, 1990.

[84]J. Rumbaugh, M. Blaha, J. Premellani, F. Eddy, and W. Lorensen. Object-Oriented Modeling and Design. Prentice Hall, 1991.

[85]A. D. Saja. The cognitive model: An approach to designing the human-computer 
interface. ACM SIGCHI, 21:365-375, 1985.

[86]P. Schnaidt and S. Reddy. Speedy and smart. LAN Magazine, pages 127-133, March 1991.

[87]D. Searls, J. Clark, D. Copron, and P. Matuszek. Managing flow of control in diagnostic expert systems. IEEE 1989 Annual Artificial Intelligence Systems in Government Conference, pages 303-310, January 1989.

[88]S. Sethi, Adarshpal. Bibliography on network management. ACM Computer Communication Review, 19(3):58-75, July 1989.

[89]Kumar Shah. Managing networks of the '90s. Data Communication, pages 127-141, December 1989.

[90]G. Sidhu, R. Andrews, and A. Oppenheimer. Inside AppleTalk, Second Edition. Addison-Wesley, 1990.

[91]Joe Skonupa. Managing the center of the universe. LAN Magazine, pages 69-75, March 1991.

[92]R. L. Spitzer, A. E. Skodol, M. Gilbbon, and J. B. Williams. Diagnostic and Statistical Manual of Mental Disorders (Third Edition). Brunner/Mazel, 1980.

[93]William Stallings. Handbook of Computer-Communications Standards, Volume 3, Second Edition. Howard W. Sams and Company, 1989.

[94]Douglas Steadman. ASN.I The Tutorial and Reference. Technology Appraisals Ltd., 1990.

[95]D.W. Straub and J.C. Wetherbe. Information technologies for the 1990s: An 
organizational : apact perspective. Communications of the ACM, 32(!1):1328-1339, November 1989.

[96]Gary Tjaden, Mark Wall, Jerry Goldman, and C.N. Jeromnimon. Integrated network management for real-time operations. IEEE Network, pages 10-15, March 1991.

[97]S. Waldbusser. Appletalk management information base. Technical Report RFC 1243, July 1991.

[98]Zheng Wang. Model of retwork faults. In B. Meandzija and J. Wescott, editors, Integrated Network Management, I, pages 345-352. Elsevier Science Publishers B.V.(North-Holland), May 1989.

[99]U. Warrier and L. Besaw. Common management information services and protocol over tcp/ip (cmot). Technical Report RFC 1095, April 1989.

[100]T. Yamahira, Y. Kiriha, and S. Sakata. Unified fault management scheme for network troubleshooting expert system (exnets). In B. Meandzija and J. Wescott, editors, Integrated Network Management, I, pages 637-646. Elsevier Science Publishers B.V.(North-Holland), May 1989.

[101]T. Yamamura, K. Yata, T. Yasushi, and H. Yamaguchi. A basic study of human error in communication network operation. In IEEE Global Telecommunications Conference and Exhibition, volume 2, pages 795-800. IEEE, Nov 1989. 


\section{Index}

DARPA

ARPA $\ldots \ldots \ldots \ldots \ldots \ldots 74,75,196$

AppleTalk $\ldots \ldots \ldots 100,126,129,196$

CMIP over TCP/IP . . . . . . . 78, 199

СMOT . . . . . . . . . . 78, 199

Defense Advanced Research Projects

Agency (DARPA) . . . . 74, 201

Exterior Gateway Prolocol (EGP)

$\ldots \ldots \ldots \ldots \ldots .994,98,203$

Ping

129,213

Request for Comments . . . . 75, 214

SGMP (Simple Gateway Management

Prolocol) $\ldots \ldots \ldots 7,78,83,214$

SNMP (Simple Network Management Proto-

col) . 78, 79, 81, 83, 85, 99, 105, 215

TCP/PP $\ldots \ldots \ldots \ldots \ldots$ 74,

75, 77, 78, 81, 87, 105, 115, 116, 216

Transmission Control Protocol (TCP)

$74,94,218$

UDP $\ldots .81,84,85,94,105,108,218$

DARPA $\ldots \ldots \ldots \ldots 7,74,104,200$

Intemet

7, 75, 87, 28, 109, 111, 146, 206

Intemet Activities Board $\quad \ldots$ 75, 190, 206
Internet Control Message Protocol (ICMP) $\ldots \ldots \ldots \ldots \ldots \ldots .993,98,206$

Internet Engineering Task Force

$\ldots \ldots \ldots \ldots \ldots$ 76, 100, 206

Internet Gateway Protocol (IGP) $\ldots .206$

Internet Protocol (IP)

74, 93, 97, 105, 187, 206

Intemet Research Task Force $\ldots .76,206$

Internet suite $\ldots \ldots \ldots \ldots \ldots 206$

Internet suite of protocols $\ldots \ldots \ldots 207$

General

ANSI $\ldots \ldots \ldots \ldots \ldots \ldots$

ASN.1 $\ldots \ldots \ldots \ldots \ldots \ldots$ 38, 48,

$50,51,66,78,82,87,109,149,196$

Abstract Syntax Notation One

48, 78, 87, 195

Abstract dala type $\ldots \ldots \ldots \ldots 49,195$

Abstract syntax $\ldots \ldots \ldots$ 48, 49, 89, 195

Agent $\ldots \ldots \ldots \ldots \ldots$ 19, 82, 195

American National Standards Institure $\ldots \ldots \ldots \ldots \ldots \ldots . . \ldots 162,196$

Backbone ......... 3, 121, 197

Baseband $\ldots \ldots \ldots \ldots \ldots$ 198, 202

Bridge . . . 15, 88, 118, 123, 144, 198 


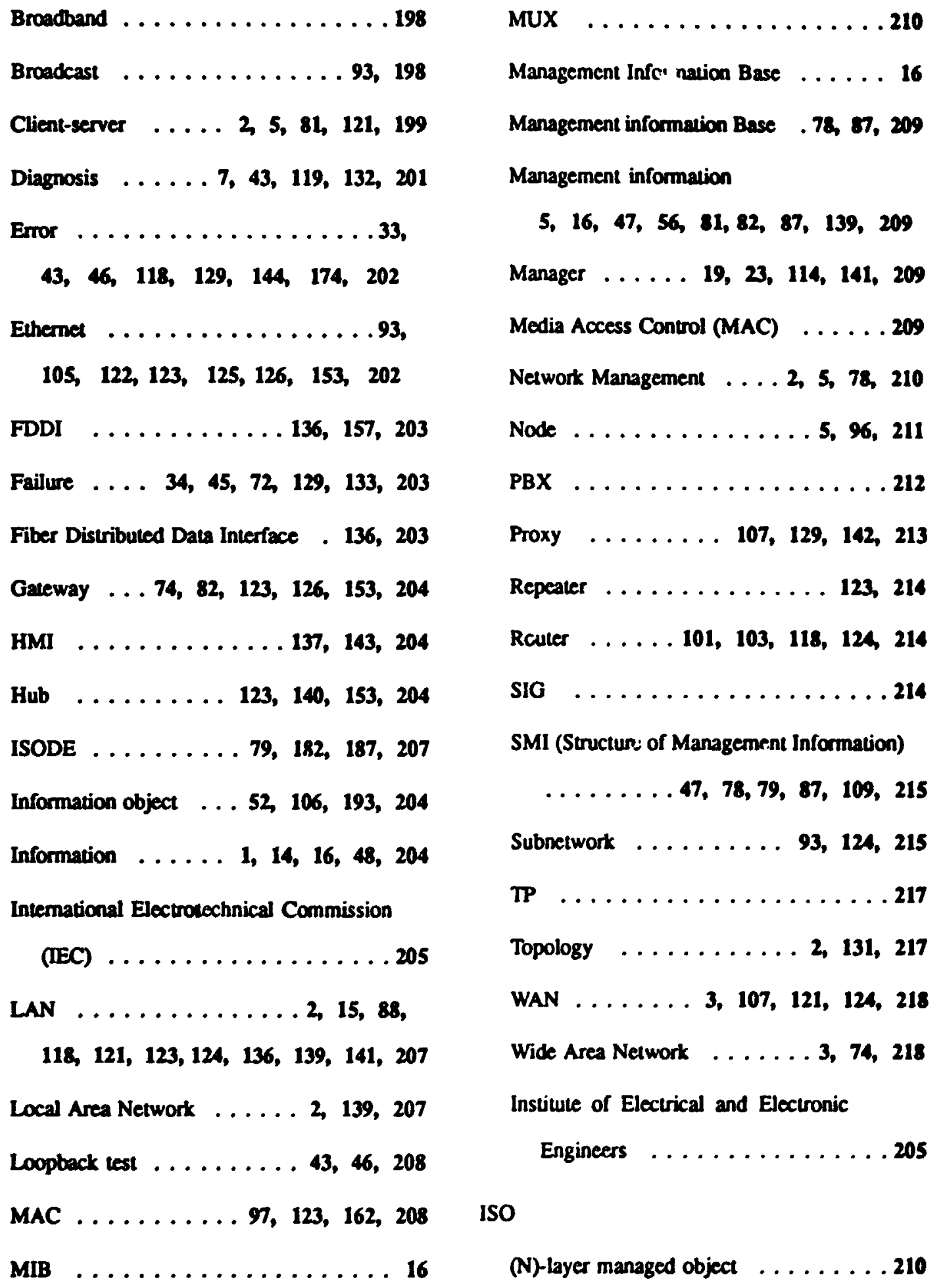


(N)-layer operation $\ldots \ldots \ldots \ldots \ldots 211$

ACSE $\ldots \ldots \ldots \ldots \ldots \ldots 195$

AE $\ldots \ldots \ldots \ldots \ldots \ldots \ldots$

AMP $\ldots \ldots \ldots \ldots \ldots 28,196$

ARF $\ldots \ldots 25,33,121,136,180,196$

ARR $\ldots \ldots \ldots \ldots 25,31,136,196$

ASE $\ldots \ldots \ldots \ldots \ldots \ldots \ldots$

AVA $\ldots \ldots \ldots \ldots \ldots \ldots \ldots$

Abnormal (lest) lermination . . . . . 195

Abstract syntax $\ldots \ldots \ldots \ldots \ldots 16$

Agent role $\ldots \ldots \ldots \ldots \ldots 20,195$

Alarm report $\ldots \ldots \ldots \ldots 37,118,196$

Alarm $\ldots \ldots \ldots \ldots \ldots 33,34,195$

Allomorphism $\ldots .58,64,65,71,196$

Associsled objects $\ldots \ldots \ldots \ldots . \ldots 196$

Asynchronous lest $\ldots \ldots \ldots \ldots \ldots$

Atribule identifier $\ldots \ldots \ldots \ldots$. . 197

Atribute type $\ldots \ldots \ldots \ldots \ldots \ldots 197$

Atribute value assertion . . 60, 197, 220

Atribute value set $\ldots \ldots \ldots \ldots$. . 197

Attribute $\ldots \ldots \ldots \ldots \ldots \ldots .5,17$,

$23,29-31,38,47,55,62,63,67,197$

BER $\ldots \ldots \ldots \ldots \ldots \ldots \ldots 198$

Backup relationships . . . . . . . . 197
Behaviour $\ldots \ldots \ldots 5$, 17, 35, 48,

$49,64,67,71,85,89,120,143,198$

CCITT . . . . . . 8, 49, 53, 68, 198

CD $\ldots \ldots \ldots \ldots \ldots 11,134,199$

CMIP $\ldots \ldots \ldots \ldots 20,105,136$, 199

CMIS $\ldots \ldots$ 20, 21, 25, 135, 182, 199

$\cos A C \ldots \ldots \ldots \ldots 10,200$

Canadian Open Systems Application Criteria

. . . . . . . . . . . 10, 198

Characteristics $\ldots \ldots \ldots \ldots 70,199$

Class-specific elements $\ldots \ldots \ldots$. 199

Comite Cunsultatif Intemationale

Telegraphique et Telephonique . . . 199

Common Management Information Protocol

$\ldots \ldots$ 20, 78, 79, 133, 135, 136, 199

Common Management Information Service $\ldots \ldots \ldots$ 20, 21, 78, 133, 135, 199

Conditional package $\ldots 68,69,111,200$

Connectionless Network Protocol (CLNP) .200

Connectionless Transport Prolocol (CLTP)

Connectivity test $\ldots \ldots \ldots \ldots 46,200$

Containment $\ldots .32,56,59,147,200$

Corporation for Open Systems (COS)

9, 200 


\begin{abstract}
Cuppration for Open Systems . . . . . 9
DIS $\ldots \ldots \ldots \ldots \ldots \ldots 11,201$

DMI $\ldots \ldots \ldots \ldots \ldots \ldots 6$ 63, 202

Data integrity test $\ldots \ldots \ldots \ldots 46,201$

Discriminator construct ... 39, 41, 201

Discriminator inpus objoct $\ldots \ldots 201$

Discriminator . . . . . . . 39, 201

Distinguished name $\ldots \ldots \ldots 60,202$

ERF

$25,38,136,202$

Encapsulation $\ldots \ldots \ldots \ldots \ldots$ 55, 202

Entity $\ldots \ldots \ldots \ldots 17,20,43,202$

Event forwarding discriminator . . . 39, 202

Event report management function (ERMF)
\end{abstract}

28,203

Event report $\ldots .25$, 38, 39, 41, 107, 202

Event .. 22, 30, 33, 41, 57, 129, 202

Fallback relationships $\ldots \ldots \ldots 203$

Fault . 17, 33-35, 43, 118-120, 129, 203

Functional Profile $\ldots \ldots \ldots \ldots 203$

Functional profiles $\ldots \ldots \ldots \ldots$. .

GDMO $\ldots \ldots \ldots \ldots \ldots \ldots .66$ 63,

$66,89,109,110,145,148,160,204$

GNMP $\ldots \ldots \ldots \ldots \ldots 134,136,204$

GosIP . . . . . . . . . .9, 204

Government OSI Profite $\ldots \ldots \ldots 204$
Government OSI Profiles $\ldots \ldots \ldots \ldots$. .

Group relationships $\ldots \ldots \ldots 204$

IEC $\ldots \ldots \ldots \ldots \ldots$

ISOREC JTC1 $\ldots \ldots \ldots \ldots \ldots$ s,

8, 9, 112, 157, 160, 161, 192, 207

Iso $\ldots \ldots \ldots \ldots \ldots \ldots$

ISP $\ldots \ldots \ldots \ldots \ldots \ldots \ldots$

Inheritance hierarchy $\ldots 57,70,148,205$

Inheritance $\ldots \ldots 57,69,70,148,204$

Intemal resource lest $\ldots \ldots \ldots$ 46, 205

International Electrotechnical Commission

Intemational Organization for

Standardization (ISO) $\ldots \ldots \ldots 205$

Intermational Organization for

Standardization ....... \& 205

International Standardized Profile (SSP) . 205

Intemational Standardized Profiles ..... 9

Intemational Telegraph and Telephone

Consultative Commituce $\ldots \ldots \ldots 8$

Intemational Telephone and Telegraph

Consultative Commillee (CCrTT) . . 206

Intrusive test $\ldots \ldots \ldots \ldots$ 45-47, 207

LCF $\ldots \ldots \ldots \ldots \ldots 25,41,207$

Log record identifier . . . . . 41, 207

$\log \ldots \ldots \ldots \ldots 207$ 


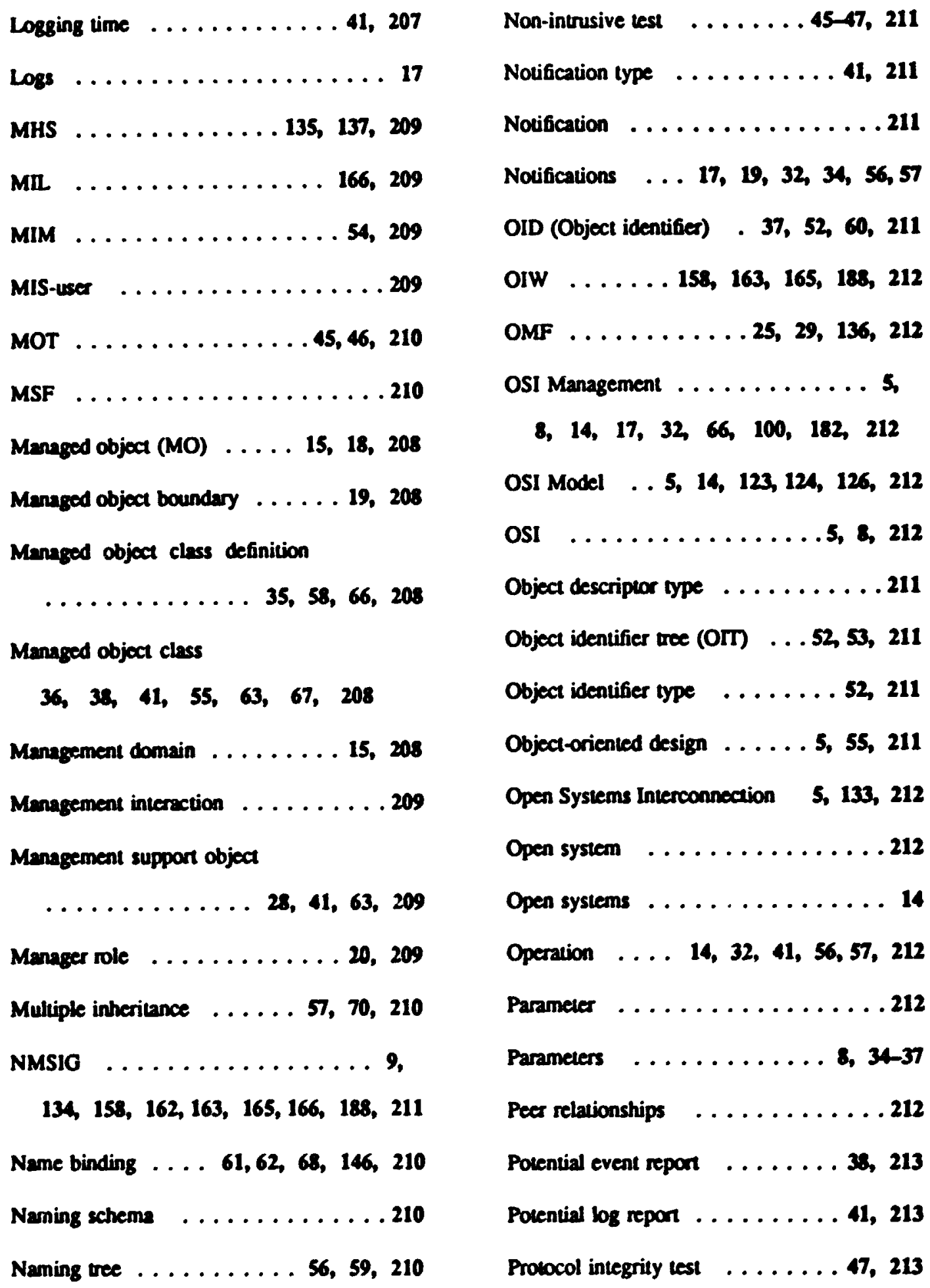


Real open system $\ldots \ldots \ldots \ldots$ 42, 213

Relative distinguished name (RDN)

$$
44,60,68,214
$$

SF

$28,63,214$

sig $\ldots \ldots \ldots \ldots \ldots \ldots \ldots$

SMAE $\ldots \ldots \ldots \ldots \ldots 138,215$

SMFA $\ldots \ldots .16,17,25-28,118,215$

SMF $\ldots \ldots \ldots \ldots 19,25-28,215$

SMO $\ldots \ldots \ldots \ldots \ldots 17,55,215$

STMF $\ldots \ldots \ldots \ldots \ldots 25,30,215$

Service rehaionships $\ldots \ldots \ldots \ldots 214$

Spocinlization $\ldots \ldots \ldots$ 55, 57, 66, 215

Status $\ldots \ldots .889,107,128,129,215$

Subclaxs $\ldots \ldots \ldots 38,57,58,69,215$

Subordinale object ..... 61, 90, 215

Supercless $\ldots \ldots \ldots \ldots$ 38, 41, 57, 215

Superior objoct $\ldots \ldots \ldots 661,147,216$

Synchronous rest $\ldots \ldots \ldots \ldots \ldots 216$

Systems Management Function (SMF) .216

Systems Management Functional Area

(SMFA) $\ldots \ldots \ldots \ldots 118,216$
Systems Management Functional Areas . 16 Systems management application entity

(SMAE) $\ldots \ldots \ldots \ldots \ldots 138,216$

Systems management function $\ldots \ldots 19$

Systems management operations ..... 17

Systems management protocol 5S, sh, 216

Systems management services $\ldots$ 19, 216

TARR $\ldots \ldots \ldots \ldots \ldots \ldots$ 4, 216

TMF $\ldots \ldots \ldots \ldots \ldots 28,42,217$

Templave $\ldots \ldots \ldots \ldots 60,67,216$

Test action request roceiver (TARR)

44, 217

Test conductor (TC) $\ldots \ldots \ldots .43,217$

Test invocalion $\ldots \ldots \ldots$ 43, 45, 217

Test objoct (TO) $\ldots \ldots \ldots \ldots$ 4, 217

Test performer $\ldots \ldots \ldots \ldots$ 43, 217

Test request $\ldots \ldots \ldots \ldots \ldots$ 4, 217

Test session $\ldots \ldots \ldots \ldots \ldots \ldots 217$

vT $\ldots \ldots \ldots \ldots \ldots$ 135, 137, 218

WD $\ldots \ldots \ldots \ldots \ldots \ldots$ 10, 218

WMF $\ldots \ldots \ldots \ldots \ldots 28,218$ 

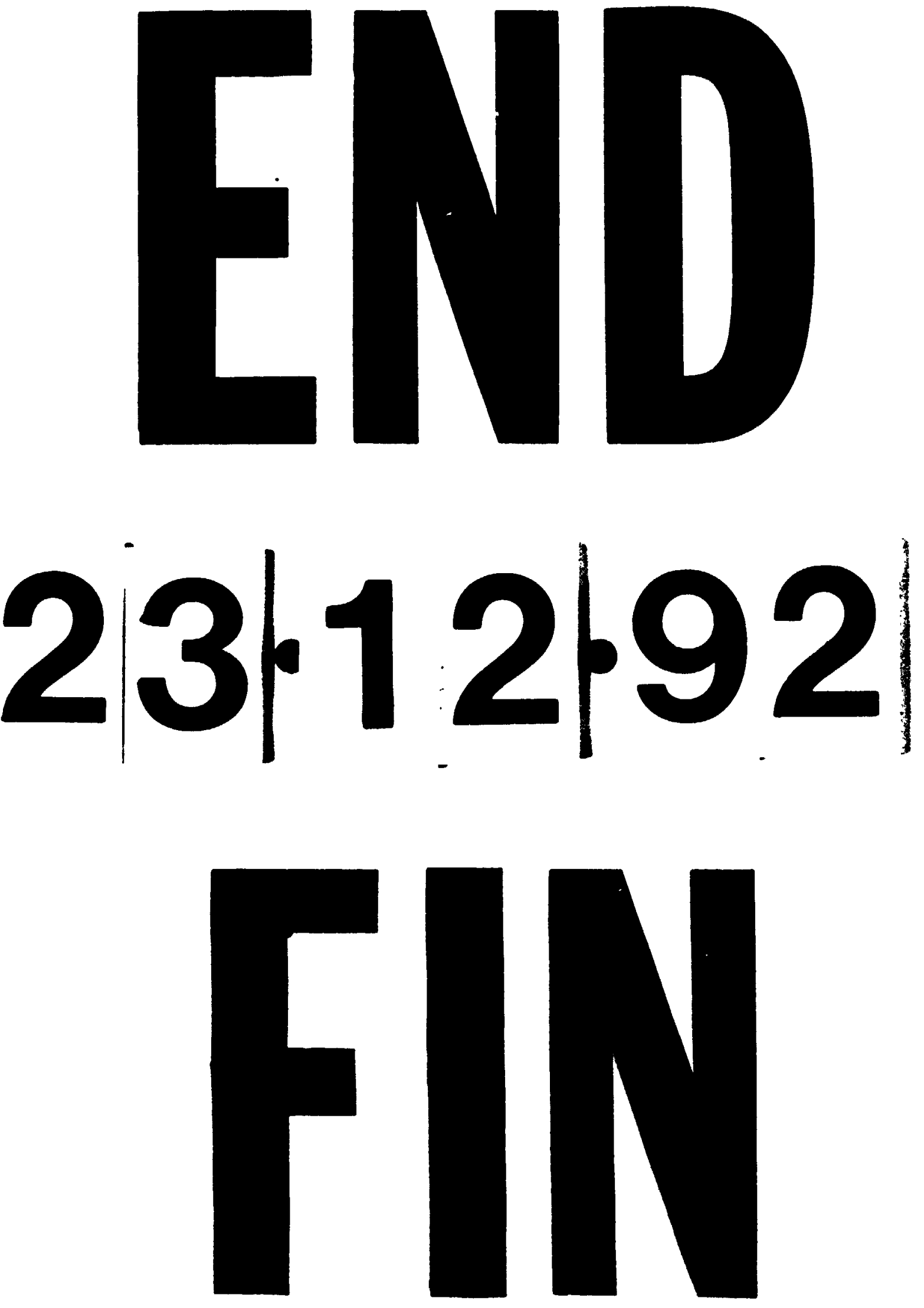